\title{
Simulation of Multi-Component Gas Flow and Condensation in Marcellus Shale Reservoir
}

\author{
Abdallah Elamin \\ West Virginia University
}

Follow this and additional works at: https://researchrepository.wvu.edu/etd

\section{Recommended Citation}

Elamin, Abdallah, "Simulation of Multi-Component Gas Flow and Condensation in Marcellus Shale Reservoir" (2013). Graduate Theses, Dissertations, and Problem Reports. 236.

https://researchrepository.wvu.edu/etd/236

This Thesis is protected by copyright and/or related rights. It has been brought to you by the The Research Repository @ WVU with permission from the rights-holder(s). You are free to use this Thesis in any way that is permitted by the copyright and related rights legislation that applies to your use. For other uses you must obtain permission from the rights-holder(s) directly, unless additional rights are indicated by a Creative Commons license in the record and/ or on the work itself. This Thesis has been accepted for inclusion in WVU Graduate Theses, Dissertations, and Problem Reports collection by an authorized administrator of The Research Repository @ WVU. For more information, please contact researchrepository@mail.wvu.edu. 
Simulation of Multi-Component Gas Flow and Condensation in Marcellus Shale Reservoir

\section{Abdallah Elamin}

Thesis submitted to the College of Engineering and Mineral Resources at West Virginia University in partial fulfillment of the requirements for the degree of

Master of Science

In

Petroleum and Natural Gas Engineering

Approved by

Ebrahim Fathi, PhD. Committee Chairperson

Samuel Ameri, Prof. Department Chairperson

Ilkin Bilgesu, PhD.

Department of Petroleum and Natural Gas Engineering

Morgantown, West Virginia

2013

Keywords: phase behavior, unconventional shale characterization, sorption kinetics, Experimental design, organic nano pores confinement and micro and nano fluidics.

Copyright 2013 Abdallah Elamin 


\section{ABSTRACT \\ SIMULATION OF MULTICOMPONENT GAS FLOW AND CONDENSATION IN MARCELLUS SHALE RESERVOIR}

By Abdallah Elamin

The Marcellus shale formation, with more than 463 trillion cubic feet (Tcf) of recoverable gas in Pennsylvania and West Virginia, will play a critical role in providing clean energy, environmental sustainability, and increased security for our nation. However, due to recent low gas prices, most of the operating companies have slowed down their activities in dry gas areas and refocused their attention in oil and condensate production from liquid-rich regions. This change in production plans requires detailed investigation of gas condensate bank developments and saturation dynamics in shale gas reservoirs that change greatly with reservoir conditions. An advanced level of understanding of the parameters affecting gas condensate phase behavior is necessary in order to make accurate predictions of these changes.

One of these parameters is the phase behavior of gas condensate in shale gas reservoirs that is significantly different than that of gas condensate as bulk in the PVT cell. It is highly affected by shale pore size distribution, gas adsorption, and water vapor saturation. Critical properties of gas condensate are also significantly influenced by shale pore size distribution, leading to changes in viscosity and formation volume calculations. In addition to that fluid composition, natural and hydraulic fractures, reservoir anisotropy, rock compressibility and number of horizontal wells and their operating conditions could also significantly impact the condensate bank development and dynamics. To quantify the importance of each one of these parameters and their interactions on dynamics of condensate bank development, an experimental design technique, Plackett-Burman design, will be practiced for two different cases (single well cylindrical model and actual Marcellus shale gas reservoir with heterogeneous porosity and permeability field). Detailed uncertainty analysis of different parameters has a significant impact on implementing the best production strategies such as bottom-hole pressures and hydraulic fracture spacing. Commercial simulators are unable to provide 
reliable predictions of condensate production rates and saturation dynamics due to lack of correct physics controlling production mechanisms in shale gas reservoirs.

In this study we will introduce a new equation of state, including the cohesive and adhesive forces due to fluid-fluid and fluid-solid interactions, and use that to develop a compositional model for gas condensate fluids in Marcellus shale gas reservoirs. A new correlation to adjust critical properties of gas condensate will also be developed based on shale pore size distribution to incorporate into the compositional simulator, CMG (GEM), to investigate the dynamics of gas condensation, and to perform sensitivity analysis on saturation profiles for different gas compositions of Marcellus from "superrich" to liquid-rich areas.

Based on our study, critical properties and phase behavior of gas condensate are distinctively different under the influence of wall effects and adsorption in organic nanopores, and also have significant effect on production strategies and stimulation design for Marcellus shale gas reservoirs. This study takes a unique approach that can be applied to commercial simulators as a modification to currently applied models without requiring rewriting or development of a new generation of simulators. 


\section{DEDICATION}

This work is dedicated to my mother and brothers. They have given me the confidence and the courage to reach all my goals.

Also, to my wife who was very patient in difficult times and very supportive for me to carry on this project. 


\section{ACKNOWLEDGMENTS}

First, I would like to thank my advisor, Dr. Ebrahim Fathi, for his commitment and his infinite help throughout my research and his insightful comments during this study. I would like to express my sincere gratitude for his patience and encouragement to me, without which this work would not have been possible. Dr. Fathi is not only my advisor, but he is also my closest friend who helped me a lot in every aspect in my life. Thank You Dr.Fathi.

Additionally, I would like to thank Dr. Ilkin Bilgesu for his valuable information and feedback.

I would like to extend my thanks to Department Chair Sam Ameri for his willingness to express and extend a warm sense of confidence towards me.

My special appreciation goes to my love, Najia, for believing in me and to my lovely daughter, Hadeel, the best thing that blessed my life.

To them, this work is dedicated.

I am extremely grateful to the entire West Virginia Petroleum Engineering faculty for their significant contribution to my academic and intellectual development. I would like also to thank my colleagues at this department not only for their constructive comments about my research but also for the wonderful time which I will never forget.

Finally, Special thanks also go to my academic advisor Ms. Amanda Ross at the Canadian Bureau for International Education for her instant monetary and moral support for my academic progress.

"In order to succeed, your desire for success should be greater than your fear of failure."

Bill Cosby 


\section{TABLE OF CONTENTS}

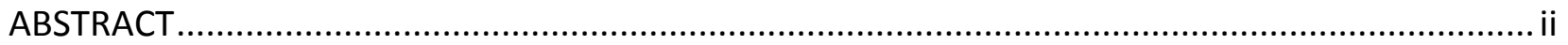

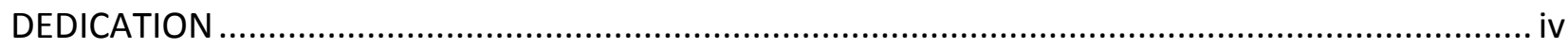

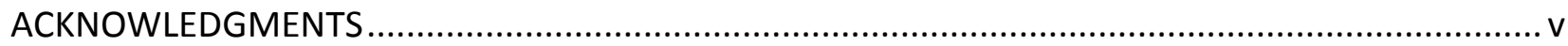

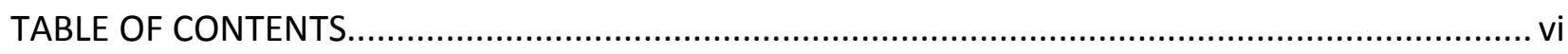

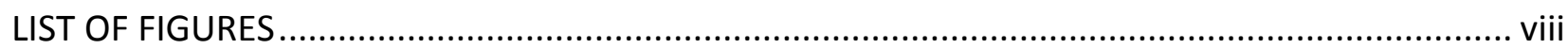

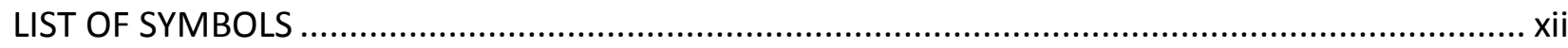

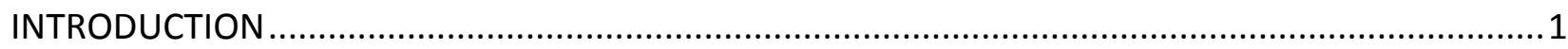

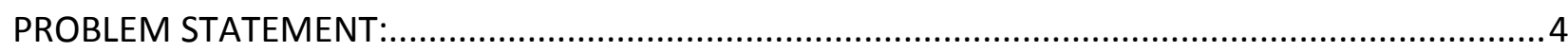

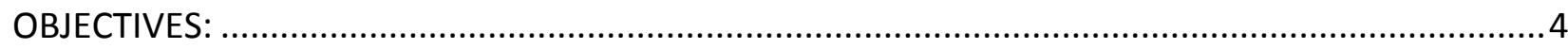

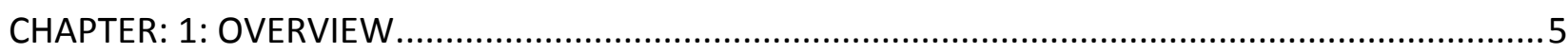

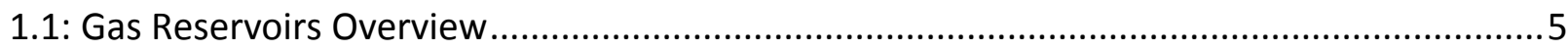

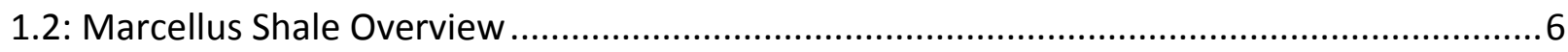

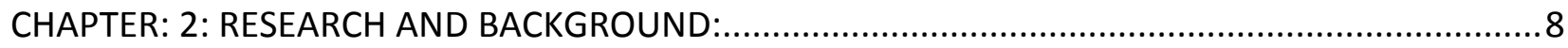

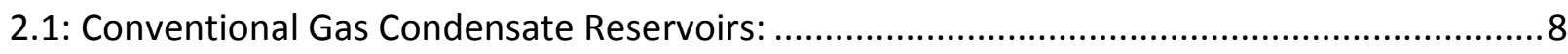

2.1.1: Characteristic of Gas Condensate Fluid Model:...........................................................

2.1.2: Characteristic of Gas Condensate Flow Model......................................................... 13

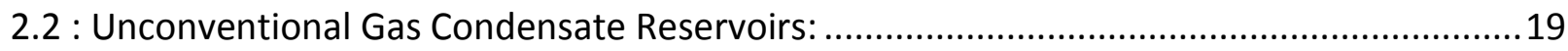

2.2.1. Imaging Techniques for Characterization of Shale Gas Reservoirs: ...............................20

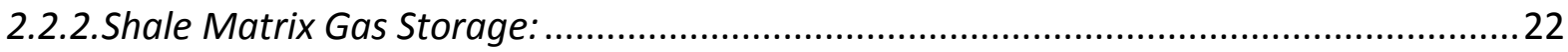

2.2.3. Confinement Effects on Local Dynamics of Shale Gas Reservoirs: ................................24

2.2.4. Adsorption Effect on the Gas Flow inside Nano-Porous Media.....................................29

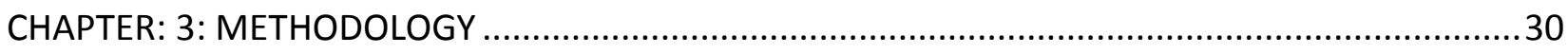

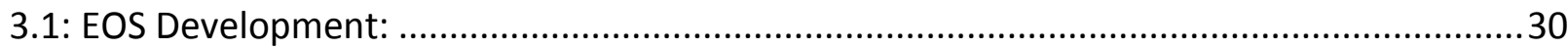

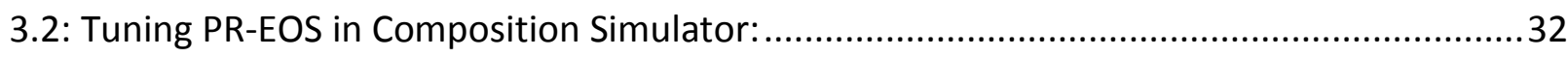

3.3: Phase Envelope Calculation of the Gas Mixture inside the Organic Nano-Porous Media: . 37

3.4: Reservoir and Fluid Characteristics Effects on the Condensation Bank Development. ......39

3.5: Operational and Completion Condition Effects on the Condensation Bank Development.39

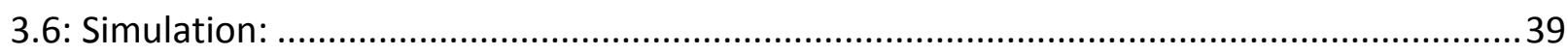

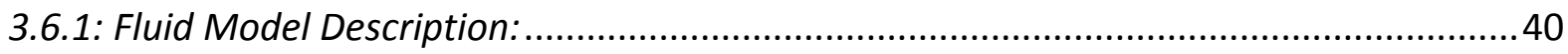




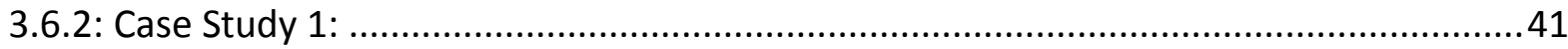

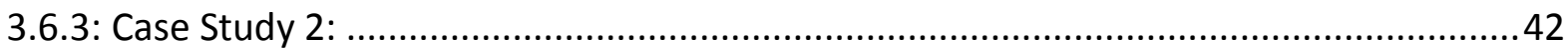

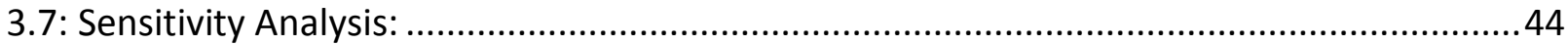

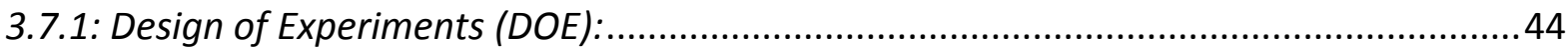

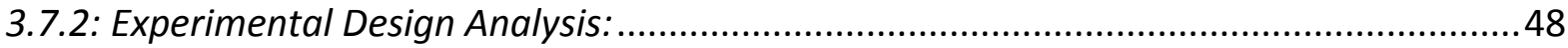

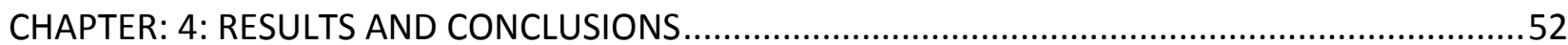

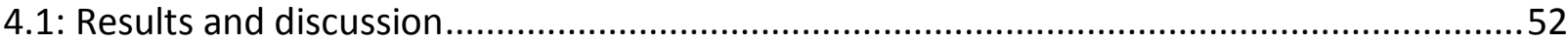

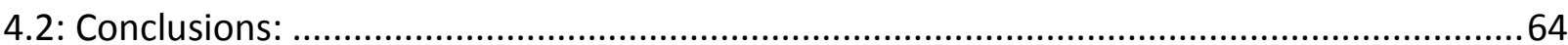

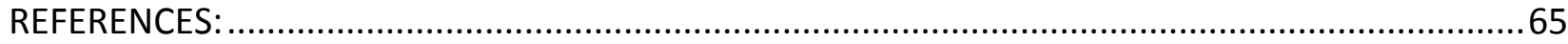

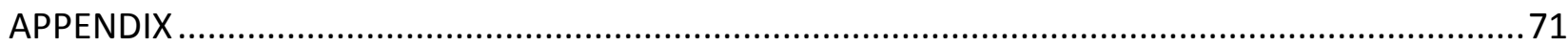

Appendix A: The CMG Software Application: .............................................................. 71

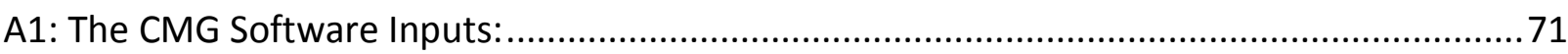

A.2: Detailed investigation of condensation build up around different wells with and without

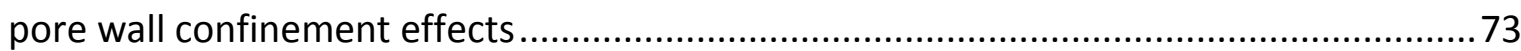

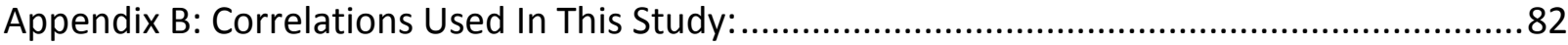

B.1: Determination of the Critical Properties Deviation Due To Pore Wall Confinement:.........82

B.2: Viscosity Correlations Of Pure Substances: (JST) ................................................... 85 


\section{LIST OF FIGURES}

Figure 1: Typical phase diagram for gas condensate reservoir.................................. 5

Figure 2: Marcellus shale distribution in the USA. [Geology, 2011] ............................. 6

Figure 3: Near-wellbore scope [Wheaton et. al, 2000]. ........................................... 9

Figure 4: Condensate profile for different gas mixture after 1-year production

[Bourbiaux, 1994].

Figure 5: The compressibility chart for multi-component gas mixture adopted from

Standing and Katz, Trans. AIME, 1942 .................................................... 12

Figure 6: Schematic gas condensate flow spatial behavior in the reservoir .................. 14

Figure 7: Schematic 3-region condensate bank development using the pressure profile

[Fan Li et al. 2005/2006] .................................................................. 15

Figure 8: The productivity profile regarding the non-darcy flow [Bourbiaux, 1994] .......... 16

Figure 9: The effect of non-darcy flow on the cumulative gas production of a fractured well [ Miskimins et al. 2005] ................................................................. 16

Figure 10: Typical relative permeability behavior in the conventional reservoirs [Fan Li et

al., 2005/2006]............................................................................. 17

Figure 11: Distribution of the organic material inside the shale matrix [Kang et al, 2011].

Figure 12: FIB/SEM picture shows cross section view of 9 shale samples adopted from Curtis et al. (2010). Dark grey areas are Kerogen and the bright grey is in organic material and the pores as black dots

Figure 13: FIB/SEM image shows a scope on nano-pores within the organic material [Ambrose et al,2010].

Figure 14: Most of porosity is related to the Kerogen. Yellow outlines are 3D Kerogen network and the red outlines are the porosity [ Ambrose, 2010].

Figure 15 : Large portion of pore volumes are associated with Kerogen [SPE-147397] 23

Figure 16: Matched the theoretical approach with experimental data 26

Figure 17: Molecular dynamics results (the empty shapes) matching with experimental data (the bolded shapes).[Singh, 2009]

Figure 18: The deviation in the critical properties of pure n-alkanes components Vs. the inverse slit width $(\mathrm{H})$ in Graphite and Mica pores [Singh 2009].

Figure 19: Curve fitting method to reach out various organic pore sizes adopted from Sapmanee (2011).

Figure 20 : Extrapolation for range of components adopted from Sapmanee (2011) ..... 29

Figure 21 : Simulation of gas flow inside the pores network (Molecular layer density for methane at $176^{\circ} \mathrm{F}\left(80^{\circ} \mathrm{C}\right)$ across the half-length of a 3.74-nm organic slit pore.). Adopted from (SPE-134583)

Figure 22: Correlation shows the outcome difference between a semi-analytical solution (dashed blue line), molecular dynamic solution (solid green line), by Singh et 
al. (2009) used as a reference, and the correction approach (solid red line) in graphs for Methane using different pore diameters.

Figure 23 : Correlation shows the outcome difference between a semi-analytical solution (dashed blue line), molecular dynamic solution (solid green line), by Singh et al. (2009) used as a reference, and the correction approach (solid red line) in graphs for Butane using different pore diameters.

Figure 24 : The percentage of critical temperature deviation from the bulk value deviation for pure components using the corrected semi-analytical solution at a range of pore sizes

Figure 25 : The percentage of critical Pressure deviation from the bulk value for pure components using the corrected semi-analytical solution at a range of pore sizes

Figure 26 : Phase envelope changes at range pore sizes using the base case fluid model. 38

Figure 27: The Two Phase envelop of the mixture [ WinProp 2012]. 41

Figure 28: The depth top grid view for the reservoir model.

Figure 29: The grid top view (3D \& 2D) of the base case model (the actual model) ...... 43

Figure 30: Determining the damage zone due to the condensate bank development using the GOR.

Figure 31: Pareto chart shows the importance of parameters evaluated on t-value. It displays a general example for seven synthetic factors (A-G) were sorted based on the chart rule.

Figure 32: The normality test of the parameters shows a normal distribution.

Figure 33: 3-D surface generated using the color variation to visualize the magnitude and trend and the parameters impact on the response value

Figure 34: 3-D surface generated using the contour lines to show the magnitude and trend and the parameters impact on the response value

Figure 35: Liquid dropout volume percentage for the base case fluid model considering the well pore approximation. 52

Figure 36: Rock-fluid graphical interpretation provided by the CMG simulator ............... 53

Figure 37: Condensation profile around well no. (1) Cylindrical Case........................... 54

Figure 38: Condensation profile around well no. (2) Unconfined case......................... 55

Figure 39: Condensation profile around well no. (4) Vertical well confined case............ 55

Figure 40: P-T phase diagram for different pore sizes ............................................ 56

Figure 41: P-T Phase diagram illustrates condensate local influence by the operation condition.

Figure 42: Analysis for 5 candidate parameters (Cylindrical Base Case Model) DesignExpert Software 59

Figure 43: Analysis for 7 candidate parameters (unconfined Base Case Model) DesignExpert Software 60 
Figure 44: Analysis for 7 candidate parameters (confined Base Case Model) DesignExpert Software

Figure 45: Interaction between parameters have the most negative impact on the condensate ring extension for unconfined case.

Figure 46: Interaction between parameters have the most negative impact on the condensate ring extension for confined case.

Figure 47 : Gas production for the base case model of the actual reservoir. 63

Figure (A1. 1 ): Marcellus structure map. 71

Figure (A1. 2): Marcellus thickness map. 71

Figure (A1. 3): Marcellus permeability map.

Figure (A1. 4): Marcellus porosity map 72

Figure (A2. $1 \mathrm{a} \& \mathrm{~b}$ ): Condensate saturation profile around all wells Run1, unconfined case and confined case respectively...

Figure (A2. 2 a \& b): Condensate saturation profile around all wells Run2, unconfined case and confined case respectively.

Figure (A2. 3 a \&b): Condensate saturation profile around all wells Run3, unconfined and confined case respectively.

Figure (A2. 4 a \& b): Condensate saturation profile around all wells Run4, unconfined case and confined case respectively..

Figure (A2. 5 a \& b): Condensate saturation profile around all wells Run5, unconfined case. and confined case respectively.

Figure (A2. 6 a \&b): Condensate saturation profile around all wells Run6, unconfined case and confined case respectively.

Figure (A2. 7 a \&b): Condensate saturation profile around all wells Run7, unconfined case and confined case respectively.

Figure (A2. 8 a \& b): Condensate saturation profile around all wells Run8, unconfined case and confined case respectively. 


\section{LIST OF TABLES}

Table 1: Marcellus Resrvoir Specifications [Roth, 2013] ....................................................... 7

Table 2: Base case fluid model with physical properties (Win Pop 2012) .................................36

Table 3: Critical parameters calculation results with new consideration for different pore sizes. 38

Table 4: Mixture components details [ Imo-Jack, 2010]..................................................... 40

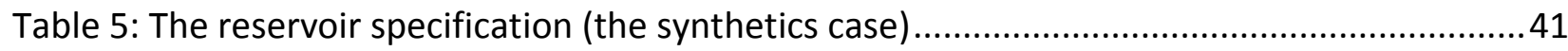

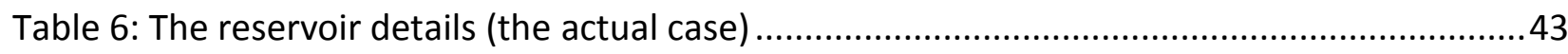

Table 7: Fluid model variation for sensitivity analysis. ......................................................45

Table 8: Candidate parameters variation ( $\mathrm{Hi}$ and low values) for the cylindrical reservoir model 46

Table 9: Candidate parameters variation ( $\mathrm{Hi}$ and low values) for the actual reservoir model. ..... 46

Table 10: Plackett-Berman matrix for seven variables, $(-1)=$ Low Value; $(+1)=$ Hi Value .............48 


\section{LIST OF SYMBOLS}

A: Helmholtz free energy.

a : Correction factor due to the internal energy.

$A_{p}$ : Transversal section area of the pore.

b : Correction factor due to the molar volume

$\mathrm{C}_{1}$ : Fitting Parameter, dimensionless

$\mathrm{C}_{2}$ : Fitting Parameter, dimensionless

G : Graphite (Mineral)

GOR : Gas to oil ration, SCF/BBls.

$\mathrm{K}_{\mathrm{rg}}$ : Relative permeability of gas phase, dimensionless

$\mathrm{K}_{\mathrm{ro}}$ : Relative permeability of oil phase, dimensionless

M : Mica (Mineral)

$\mathrm{m}$ : Mass lbs

$M_{w}$ : Molecular weight, g/g-mole

$\mathrm{n}$ : Total number of components

$\mathrm{P}$ : Average Reservoir Pressure (psi)

Pc : Critical Pressure of the gas mixture.

$P_{c b}$ : Critical Pressure at Infinite Slit Width, Kelvin

$P_{p c}$ : Pseudo critical pressure of the gas mixture.

$P_{c i}:$ Critical Pressure of the a single component (i).

$\mathrm{P}_{\mathrm{cp}}{ }^{*}$ : Critical Pressure at nanoscale Slit Width, atm

$P_{d}$ : Dew Point Pressure .

$P_{\mathrm{i}}$ : Initial Reservoir Pressure.

$\mathrm{P}_{\mathrm{pr}}$ : Pseudo-reduced pressure.

$P_{r}$ : Reduced Pressure
$\Delta \mathrm{P}_{\mathrm{c}}{ }^{*}$ : Relative Deviated Critical

Pressure, dimensionless

R : Gas Universal Constant, J/K-mole

$r_{p}$ : Pore radius.

$\mathrm{S}_{\mathrm{c}}$ : Oil critical saturation.

$\mathrm{S}_{\mathrm{o}}$ : Oil Saturation.

$\mathrm{T}$ : Average Reservoir temperature $(\mathrm{F})$.

$T_{c}$ : Critical Temperature of the gas mixture.

$T_{c b}$ : Critical Temperature at Infinite Slit Width, Kelvin

$T_{p c}$ : Pseudo critical Temperature of the gas mixture.

$T_{c i}$ : Critical Temperature of the a single component (i).

$\mathrm{T}_{\mathrm{cp}}{ }^{*}$ : Critical Temperature at nanoscale Slit Width, Kelvin

$\mathrm{T}_{\text {pr: }}$ : pseudo-reduced temperature.

$T_{r}$ : Reduced Temperature.

$\Delta \mathrm{T}_{\mathrm{c}}{ }^{*}$ : Relative Deviated Critical Pressure, dimensionless

$\mathrm{V}$ : Volume of the fluid container

$\mathrm{V}_{\mathrm{pg}}$ : Gas pore velocity

$\mathrm{V}_{\mathrm{cm}}$ : Critical molar volume (cc./g-mole).

W: Volume taken up by the adsorbed phase

$y_{i}$ : Mole fraction for pure component (i).

Z : Gas compressibility factor 
$\alpha$ : the amount adsorbed

$\omega$ : acentric factor

$\sigma_{g o}:$ interfacial tension (IFT)

$\rho_{r}$ : The reduced fluid density.

$\rho_{\mathrm{s}}$ : The adsorbed phase density

$\mu_{\mathrm{f}}$ : Fluid viscosity.

$\mu_{\mathrm{g}}$ : Gas viscosity

$\mu^{*}$ : Low-pressure Viscosity, Pa-s

$\xi$ : Viscosity parameter 


\section{INTRODUCTION}

Many shale gas basins that have been under development for gas production are now considered for gas condensate production from liquid rich regions such as Eagle Ford and Barnett in Texas, Haynesville in Texas-Louisiana, Woodford in Oklahoma and Marcellus shale in Pennsylvania [U.S. EIA, 2011] and [Marcellus, 2012]. However, this change in production plans requires detailed investigation of gas condensate bank developments and saturation dynamics in shale gas reservoirs. Conventional gas condensation has been characterized as significant loss of well deliverability, when the flowing bottom hole pressure drops below the dew-point, due to the reduction in gas permeability. This phenomenon in conventional gas-condensate wells has been investigated by several authors in detail using pressure, volume and temperature (PVT) properties, field data and numerical simulators using different equation of states [Bengherbi et al, 2002, Kniazeff et al, 1965 , Muskat et al, 1936, and Zhilin et al, 2007]. In unconventional shale gas reservoirs, however, only a few exploratory studies are available on impacts of reservoir rock and fluid properties on condensate bank developments and its effect on well deliverability [Devegowda, 2012, Orangi, 2011, and Zarragoicechean, 2004 ]. This is due to the fundamental level questions, and often uncertainties, related to the total amounts and spatial distribution of original fluids in the reservoir, their thermodynamic states (i.e., absorbed, adsorbed, or free) and, finally, the mechanisms of their transport under the reservoir conditions [Akkutlu et al, 2012].

Shales are relatively low porosity and ultra-low permeability; in addition, they consist of pores with a wide range of sizes, which often leads to multi-modal pore size distribution. In general shale gas reservoirs can be characterized as dual porosity continua, where shale permeability is associated with inorganic matrix having relatively large irregularly-shaped pores and fractures, whereas molecular phenomena (diffusion and nonlinear sorption) are with the Kerogen pores [Akkutlu et al, 2012]. Kerogen is an organic nano-porous material that has been dispersed within inorganic matrix of shales. According to International Union of Pure and Applied Chemistry (IUPAC) pore size classification, Kerogen pores fall in micro-pores range with sizes less than $2.0 \mathrm{~nm}$ and 
meso-pores with sizes in the range 2-50 nm, with the average kerogen pore size typically below $10 \mathrm{~nm}$ [Adelola et al 2011, Ambrose et al, 2010]. At this scale, phase behavior and interfacial dynamics of fluids are highly affected by pore wall confinements and specific surface areas that highly influence the fluid density, viscosity, formation volume factor and ultimately fluid flow and transport in these tight formations [Campos et al 2009, Rahmani, 2012, and Sudhir, 2009 ]. Even though the multi-scale natural fractures present in the shale matrix can help to increase the specific surface areas, horizontal wells and multi-stage hydraulic fracturing technology are essential to make the production from these tight formations economically viable, providing enough connectivity with natural fractures [Arukhe et al 2009, Cheng, 2012 ].

In spite of the fact that a vast literature exists studying the multi-phase flow behavior of a single fluid flow and phase coexistence, the Special flow pattern ( free, adsorbed and absorbed phase ) for gas transport presented in nano-porous media cannot be explained by the traditional equations of state. In this study the pore size effect and the adsorption effect are compiled throughout an analytical approach to correct the fluid properties, specifically critical temperature and critical pressures of gas mixture components. On the basis of theoretical analysis following in chapter 3, a new equation of state is established that best describes the thermodynamics of fluids in unconventional shale reservoirs. By utilizing the analytical solution with the adsorption effect of the pore walls, more realistic phase behavior diagram for the fluid inside nanoporous media was generated.

Next the new equation of state is implemented in a compositional reservoir simulator (CMG-GEM) to investigate the impact of Marcellus shale gas reservoir rock and fluid properties, operational conditions and also reservoir development plan, i.e. bottom hole pressures, number of horizontal wells and hydraulic fracturing stages, on condensate bank development and saturation dynamics. For this purpose Marcellus shale gas reservoir " $\mathrm{A}$ " is considered and a systematic approach is conducted using both single well radial grid system and actual reservoir model with Cartesian grid system. In order to find the parameters that have the most impact, "heavy-hitters," on condensate 
bank development and saturation dynamics, first screening analysis is performed using an experimental design technique, Placket-Burman design, on single well cylindrical model. Next, comprehensive analysis is performed to understand the non-linear behavior of the "heavy-hitters" obtained including micro-pore size effect. The pore size effect in Marcellus shale gas reservoir is incorporated in compositional simulator using reservoir fluid critical properties corrected for nano-scale pore wall confinements in shale gas reservoirs, assuming $10 \mathrm{~nm}$ average pore size distribution. Pareto charts and Normal plot of standardized effects have been used to analyze the degree of importance and correlations between different factors and damage radius, i.e. high condensate saturation zone. 


\section{PROBLEM STATEMENT:}

The gas condensate bank and saturation profiles in shale gas reservoirs cannot be correctly modeled using commercial simulators due to the lack of correct physics controlling these mechanisms. Recent experimental and numerical studies have shown that presence of organic nano-pores can significantly affect thermodynamics and phase behavior of the fluids in unconventional shale reservoirs. That is not only because of the nano-pore wall confinement effect but also due to the physical adsorption effect. Other factors such as reservoir characteristics and the operation condition are also important parameters that can have significant impact on gas condensate bank development and saturation profiles. In this study we aimed to develop a new equation of state considering the nano-pore size and adsorption effects to study the thermodynamics of the reservoir fluids and then quantify the importance and the effects of different rock properties and operational conditions on gas condensate developments.

\section{OBJECTIVES:}

$\checkmark$ Addressing aforementioned problems and providing novel solutions for them by developing a new equation of state including adsorption and nano-pore size effects. Calculate a new saturation envelope based on new equation of state to correctly describe the fluid thermodynamics in unconventional shale gas reservoirs.

$\checkmark$ We further quantify the magnitude and importance of different parameters affecting gas condensation in heterogeneous anisotropic Marcellus shale gas reservoir using proper experimental design technique. These are fluid properties, reservoir characteristics (e.g. natural fracture density, anisotropy factor, etc.) and operation parameters (e.g. hydraulic fracture spacing, bottom hole pressure, etc.).

$\checkmark$ Finally a unique approach will be presented to answer fundamental questions on WHERE and WHEN exactly condensation happens. Is it happening inside the organic or inorganic materials? And when it exactly starts to develop? 


\section{CHAPTER: 1: OVERVIEW}

\section{1: Gas Reservoirs Overview}

Based on the phase diagram characteristics of the fluids at the reservoir condition, gas reservoirs are classified as retrograde gas-condensate, near-critical gascondensate, wet gas, and dry gas. In this study the scope was on the condensate reservoirs in which the fluid temperature at the reservoir condition lies between the cricondentherm and the critical fluid temperature as it has been shown in Figure 1. In this figure the depletion mechanism between the reservoir condition and the separator condition is also illustrated through a pressure path from the initial reservoir condition to the bottom hole flowing pressure at isothermal condition. At any point below the dew point pressure on this path, the amount of liquid dropped out can be estimated from the quality lines.

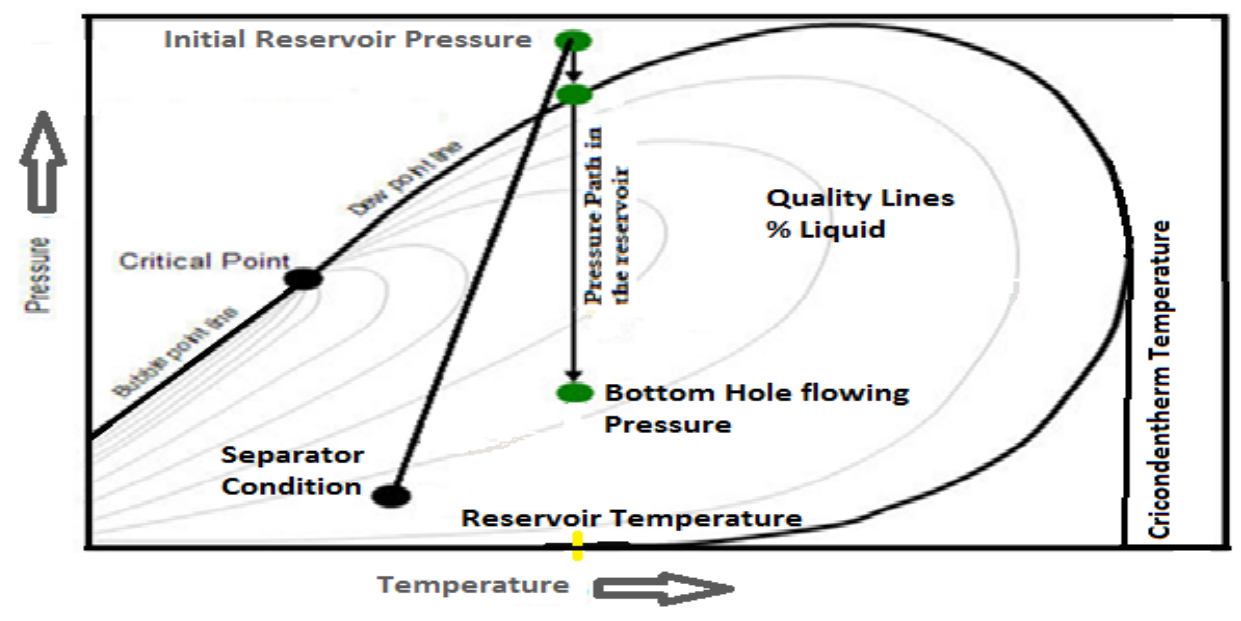

Figure 1: Typical phase diagram for gas condensate reservoir.

The production optimization techniques in the condensate reservoirs are more difficult than other types of gas reservoirs. Since the reservoir fluid is rich in heavy components, as reservoir pressure hits the dew point, the heavy components will no longer be produced at the surface; thus, the liquid phase will start building up inside the reservoir and especially around the wellbores. This stage can be recognized from the sudden increase in the gas oil ratio (GOR) curve recorded at the wellhead. This 
phenomenon is called condensate blockage or condensate banking. It severely affects the production from these reservoirs. Many factors can be involved in this phenomenon like the fluid properties, the reservoir parameters and operation conditions, which are going to be discussed in details in this study.

\section{2: Marcellus Shale Overview}

The Marcellus Shale, as indicated in the figure below, is a black shale formation deposited over 350 million years ago. It is located mostly as deep as $7000 \mathrm{ft}$, extending underground to cover an area from West Virginia in the south to New York in the north. The area covered by Marcellus is approximately 95,000 square miles. The Middle Devonian Marcellus of the Appalachia is the largest of the identified North American shale gas plays, underlying the largest gas market. Due to its potential, the Marcellus is considered to be one of the hottest spots in the North American plays [Geology, 2011]. The US geological survey announced an educated guess about Marcellus energy (initial gas in place) estimates to be around IGIP $=1500000$ Billion cubic feet (bcf), with a Recovery factor $(R F)=23.3 \%$. As for the well deliverability, well is estimated to produce at 25 Million cubic feet (mmcf) per day that leads to early reserves of 3.75 bcf per well [Roth, 2013]. Table 1 provides tabulation of Marcellus shale reservoir specification.

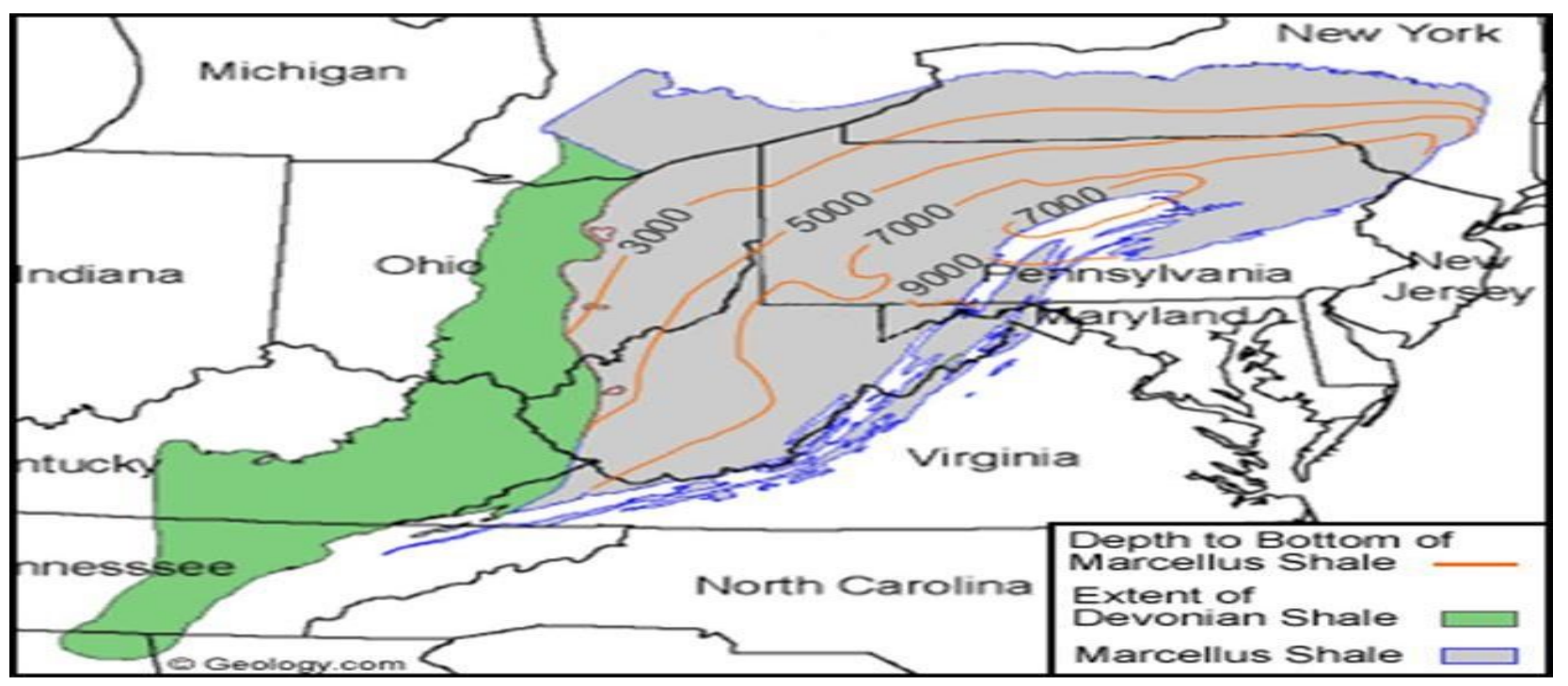

Figure 2: Marcellus shale distribution in the USA. [Geology, 2011]. 
Various completion techniques such as stimulation, horizontal drilling and hydraulic fracturing have been introduced to the oil and gas industry to create an effective connection between the well and the targeted hydrocarbon-containing formation, thereby providing a free pathway for the gas to be produced. Horizontal drilling approach allows for a greater contact length between the wellbore and the producing formation than is traditionally achieved through vertical drilling. In unconventional shale reservoirs due to ultra-low permeability of the formation, besides the horizontal well drilling technology, special techniques like hydraulic fracturing are also required to increase the exposure area of the pay zone, thus increasing the volume of hydrocarbon to be recovered. Orangi (2011) in his study showed that the surface area of the contact between the fractures-reservoir and fracture conductivity have the major impact on the productivity profile from gas condensate reservoirs where the cumulative gas production rises significantly increasing the exposure area.

In this study the effect of different stimulation and operation strategies and petrophysical reservoir parameters on the gas condensate reservoirs' potential has been inspected.

\begin{tabular}{|c|c|}
\hline Age: Middle Devonian, 385 MYA & Matrix Permeability (nD): 1,000 \\
\hline Lithology: Argillaceous Mudstone & Pressure Gradient (psi/ft): 0.4 \\
\hline Total Area Size (sq mi): 95,000 & Clay Content (\%): 50 \\
\hline Total Gas (tcf): 1,500 & Silica/Calcite/Carbonate (\%): 50 \\
\hline GIP (bcf/sq mi): 200 & Adsorbed Gas (\%): 50 \\
\hline Producible Gas (tcf): $356 t$ & Matrix Permeability $(n D): 1,000$ \\
\hline Avg. Well Depth (feet): 7,000 & Pressure Gradient (psi/ft): 0.4 \\
\hline Thickness (feet): 350 & Clay Content (\%): 50 \\
\hline \multicolumn{2}{|l|}{ Horizontal Well Cost (\$M): 3.5} \\
\hline \multicolumn{2}{|l|}{ Average EUR: 3.75} \\
\hline \multicolumn{2}{|l|}{ Pressure (psi): 4,000} \\
\hline \multicolumn{2}{|l|}{ Temperature (F): 130} \\
\hline \multicolumn{2}{|l|}{ Ro: 1.25} \\
\hline \multicolumn{2}{|l|}{ Total Organic Content, TOC (\%): 3.25} \\
\hline Porosity (\%): 8.0 & \\
\hline
\end{tabular}

Table 1: Marcellus Resrvoir Specifications [Roth, 2013] 


\section{CHAPTER: 2: RESEARCH AND BACKGROUND:}

\section{1: Conventional Gas Condensate Reservoirs:}

The gas behavior is different under different PVT. Attempts in the laboratories to mimic the real life reservoir behavior might also have limited success due to the complicated dynamic interaction between the mechanical, physical and chemical processes. In the field the condensation process is considered to be at an isothermal condition; thus, at a perfect condition, the reservoir gas converts to liquid due to the reduction in pressure, which results from the huge change in the pore volume offered to the hydrocarbons during the depletion process. However, in the case of wet gas reservoirs, the condensation could happen during the migration of the gas towards the surface because the pressure at the surface is very low compared to the reservoir. This case is all dependent on the operation conditions, like the wellhead pressure set point, which can be adjusted. On the other hand, in the condensate gas reservoirs, since this process happens completely inside the reservoir, the two major factors believed to be the most important in this process are the reservoir pressure profile and the richness of the fluid mixture. Those were mostly discussed in the literature.

The pressure profile is potentially influenced by the reservoir characteristics and is directly linked with the trend of the liquid drop-out inside the reservoir. The pressure gradient is higher near higher permeability and porosity regions such as the sand face at the wellbore and the fracture face, and decreases as the distance increases from the wellbore. The accumulation of the gas liquid around the wellbore in retrograde gas reservoirs is usually the main reason for productivity loss from those wells. Figure 3 shows a clear agreement between the liquid build-up profile around the wellbore and the pressure decline curves inside the reservoir [Wheaton et. al, 2000].

To gain a comprehensive explanation of the role played by fluid nature in the condensate bank development in the gas condensate reservoirs, different aspects need to be considered in order to establish the accurate relationship between the reservoir pressure and fluid-rock and between the reservoir pressure and the fluid-fluid 
interactions during the reservoir life. This will be discussed in detail in the next few paragraphs.

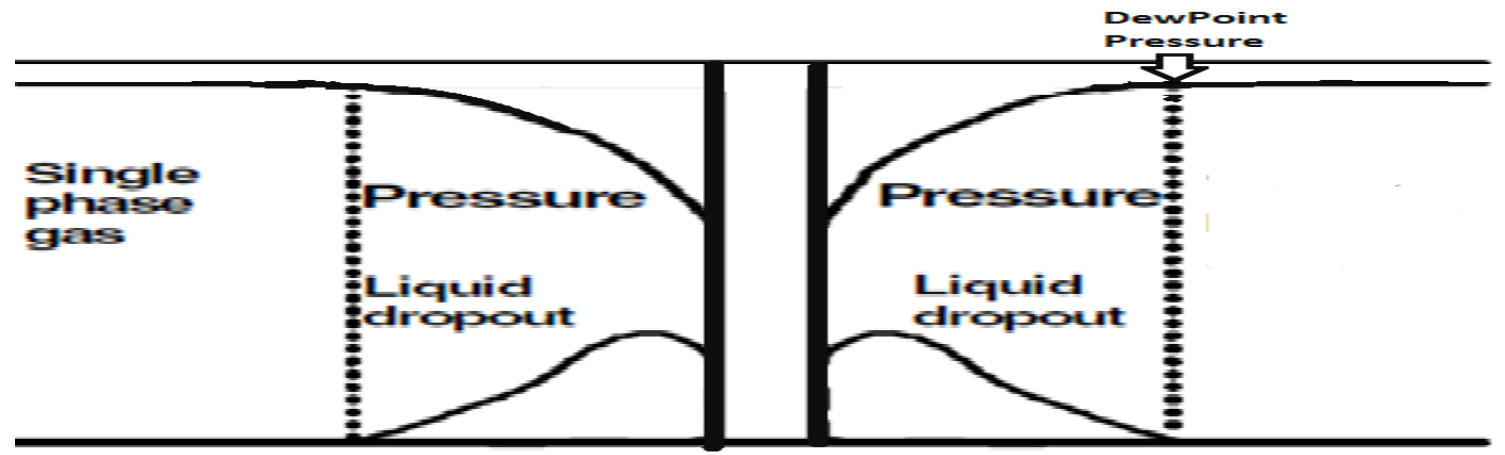

Figure 3: Near-wellbore scope [Wheaton et. al, 2000].

\subsection{1: Characteristic of Gas Condensate Fluid Model:}

Even though the behavior of the fluid under the reservoir condition might be somehow different from the laboratory experiments, the phase diagram obtained from laboratory PVT data worked just fine in studying thermodynamics of fluids in conventional reservoirs. Using laboratory investigation of phase behavior, hydrocarbon reservoir fluids are divided into five distinct groups: black oil, volatile oil, wet gas, dry gas, and condensate gas [McCain, 1990]. The phase envelopes of the gas mixtures in hydrocarbon reservoirs are smaller than that of oil. The critical point is located far down the left-hand side of the envelope and is close to the critical point of the pure light components [McCain, 1990]. In this thesis the focus is on the condensate gas reservoir or what it is called, retrograde gas reservoirs. These hydrocarbons change from the state of gas to the liquid phase when the reservoir pressure drops below the dew point pressure. This phenomenon draws the researchers' greatest attention over the recent few years because of the more valuable production and more challenging productivity problems associated with it. In few words, in this review the majority of the studies on the condensate behavior inside the reservoirs focused on the most important properties of the fluid acting inside large pores like the composition, the compressibility and the fluid viscosity. 


\section{The Effect of Reservoir Fluid Composition on Condensate Bank Development}

The percentage of the heavy components in the reservoir fluid is a very important factor controlling the reservoir potential and fluid behavior in the condensate gas reservoirs. It was mentioned by Bengherbia (2002) that upon the fall of the bottom hole flowing pressure below the dew point, the gas production decreases faster as the fluid is richer. For the pace of the condensate build up profile, which is illustrated in Figure 4 below for one year production, the same scenario happens [Bourbiaux,1994]. Moreover, the severity of other phenomena like the segregation could be more related to the richness of the fluid in the conventional reservoirs. Gravity segregation is a spatial and temporal phenomenon that has been studied intensively by different research groups. Spivak (1974) showed earlier that the tendency of the fluid to segregate is directly proportional to the reservoir permeability, fluid density and mobility ratio which are significantly influenced by production rate. A recent work by Bourbiaux, (1994) reported the segregation of the fluid as a function of the distance too, if the reservoir fluid is rich and low interfacial condition is met during the depletion. The segregation happens in the large pores that distinctly allow the separation of the condensed phase from the gas phase. This phenomenon is just like the skin effect around the wellbore in the way it decreases the permeability thus productivity of the well. To conclude, involving the reservoir fluid components and its composition does help analyzing the condensate bank development issues related to the condensate reservoirs.

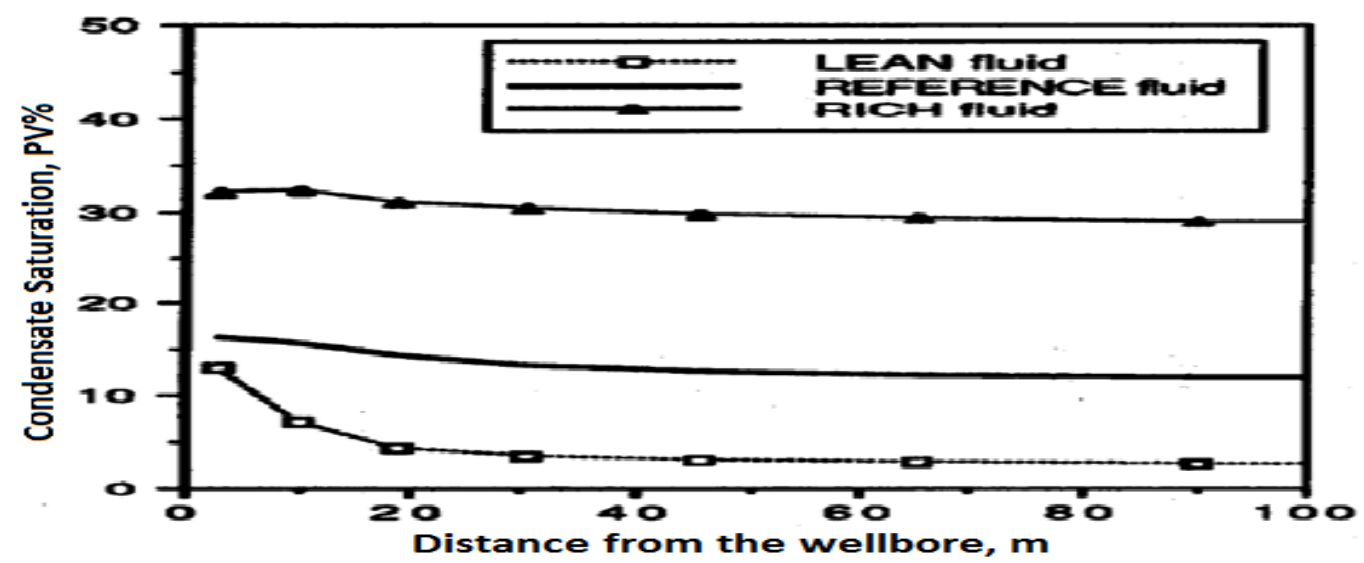

Figure 4: Condensate profile for different gas mixture after 1-year production [Bourbiaux,1994]. 


\section{Determination of Gas-Liquid Compressibility Factors:}

For a multi-component gas mixture, the gas compressibility factor, "Z-factor," is not a constant variable rather it has to be measured using the PVT-experiment because it varies with the change in gas composition, temperature and pressure [McCain, 1990]. The gas compressibility factor is explicitly expressed in most engineering studies of multi-components gas properties in terms of the pseudo-reduced pressure $\left(P_{p r}\right)$ and pseudo-reduced temperature $\left(T_{p r}\right)$. That implies using the pseudo critical properties of the gas instead of the actual properties of the gas mixture [McCain, 1990]. The use of these pseudo properties with the chart of the generalized gas compressibility factor presented by Standing et. al. (1942), can guarantee sufficient estimation accuracy for most engineering purposes. Figure 5 shows the mentioned chart of the Z-factor determination. To use this chart, those dimensionless terms have to be calculated from the following equations:

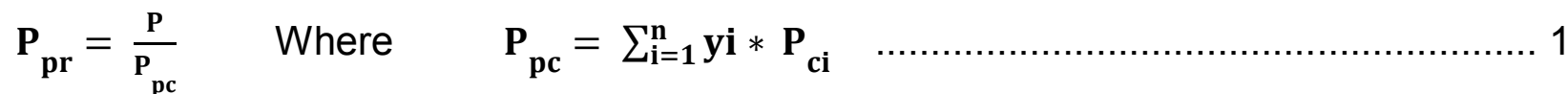

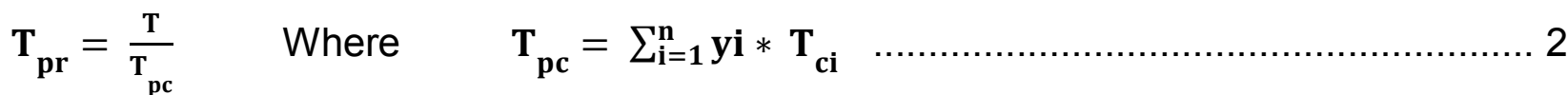

Where $P$ is the reservoir pressure, $P_{c}$ and $T_{c}$ are the critical pressure and temperature of pure component " $\mathrm{i}$ " in the gas mixture, " $\mathrm{y}$ " is the mole fraction of "ith, component in the gas mixture, $\mathrm{P}_{\mathrm{pc}}$ and $\mathrm{T}_{\mathrm{pc}}$ are pseudo critical pressure and temperature of the gas mixture and finally $\mathrm{P}_{\mathrm{pr}}$ and $\mathrm{T}_{\mathrm{pr}}$ are the reduced pressure and temperatures assigned to the gas mixture to obtain the mixture gas compressibility factor. 


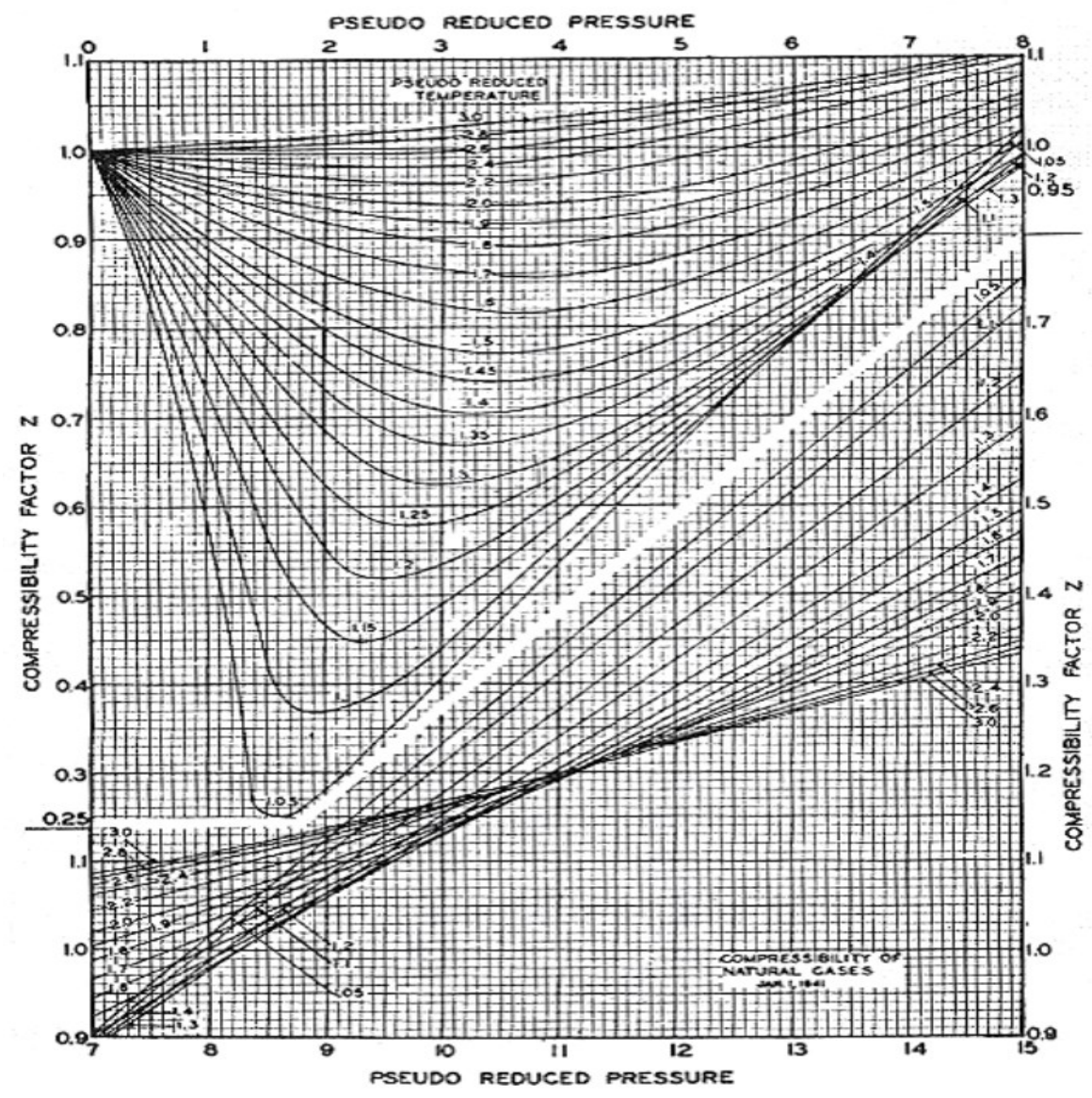

Figure 5: The compressibility chart for multi-component gas mixture adopted from Standing and Katz, Trans. AIME, 1942

\section{Determination of the Gas Mixture Viscosity}

Being a function of the pressure, temperature, and the density, the internal and external friction of the two phase flow in gas condensate reservoirs have a great impact on the gas productivity. In general, viscosity is defined as the resistance of fluid to flow that can be expressed as the ratio of shear stress to shear rate that is a property of fluid. Different solutions have been developed and applied in the industry throughout the history to estimate the viscosity of the reservoir fluid. The most popular ones were CarrKobayashi-Burrows's method in which graphical correlations were used for the viscosity estimation; Lee-Gonzalez-Eakin's method which presented a semi-empirical relationship for calculating the viscosity of the natural gases; and finally, Jossi, Stiel and Thodos (JST) and its modification of Pedersen (1987). These are the most widely used correlations by engineering applications for determining mixture viscosity using the low- 
pressure mixture viscosity. The last is used in this study as an integrated application in the CMG software. The details of this method is presented in the appendix B2 through equations (B.2.1-2), however, the main catch is viscosity highly related to critical properties of gas components.

\subsection{2: Characteristic of Gas Condensate Flow Model.}

The gas-condensate flow behavior is a complex process because of the participation of the local and temporal parameters in the driving mechanism of the condensate inside the porous media. Examples of these parameters are the changing of the liquid drop-outs saturation and buildup profiles with the time, as well as the transitioning of the fluid composition during the reservoir depletion process. The liquiddrop out in the gas phase can form a barrier-like condition around the wellbore or deep inside the reservoir that obstructs the gas flow toward the wells which in turn dramatically reduces the productivity. Also, the change in the fluid composition as the fluid becomes leaner during the production affects the flow in terms of the relative permeability. The relative permeability to the gas $\left(\mathrm{K}_{\mathrm{rg}}\right)$ can be severely reduced due to the increase of the interfacial tension between the liquid drop out and the flowing gas inside the pores. Moreover, the changes of flow pattern from laminar to turbulent due to the local condition around the wellbores can also lead to significant change in flow patterns. More discussion about these fundamental issues will be contained further on in this document.

\subsubsection{Condensate Bank Dynamics within the Reservoir:}

The condensate behavior in the condensate bank, during the reservoir life, usually follows the simple 3-region model that was proposed by Fevang and Whitson, 1996. As illustrated in figure 6 and figure 7 , the condensation profile can be analyzed according to the local saturations as follows:

Region 1: This region is the closest to the wellbore. Here the condensation saturation often exceeds its critical value at some point through the production time 
because of the severe reduction in the pressure. Both gas and liquid phases can flow simultaneously (at different velocities) in this region.

Region 2: It is located right behind the region (1) according to the condensation saturation. The size of this region is changing with time, as region (1) expands along with the depletion time. In this region the condensation behavior ordinarily resembles the static model. The liquid drop out at this zone accumulates until zone (1) takes over the region.

Region 3: This region is the farthest away from the wellbore that indicates the initial saturation condition of the reservoir at early life. At this time the reservoir pressure is commonly still high enough to maintain a value above the dew point pressure of the fluid system. The flow characteristic in this region is most likely single-phase behavior.

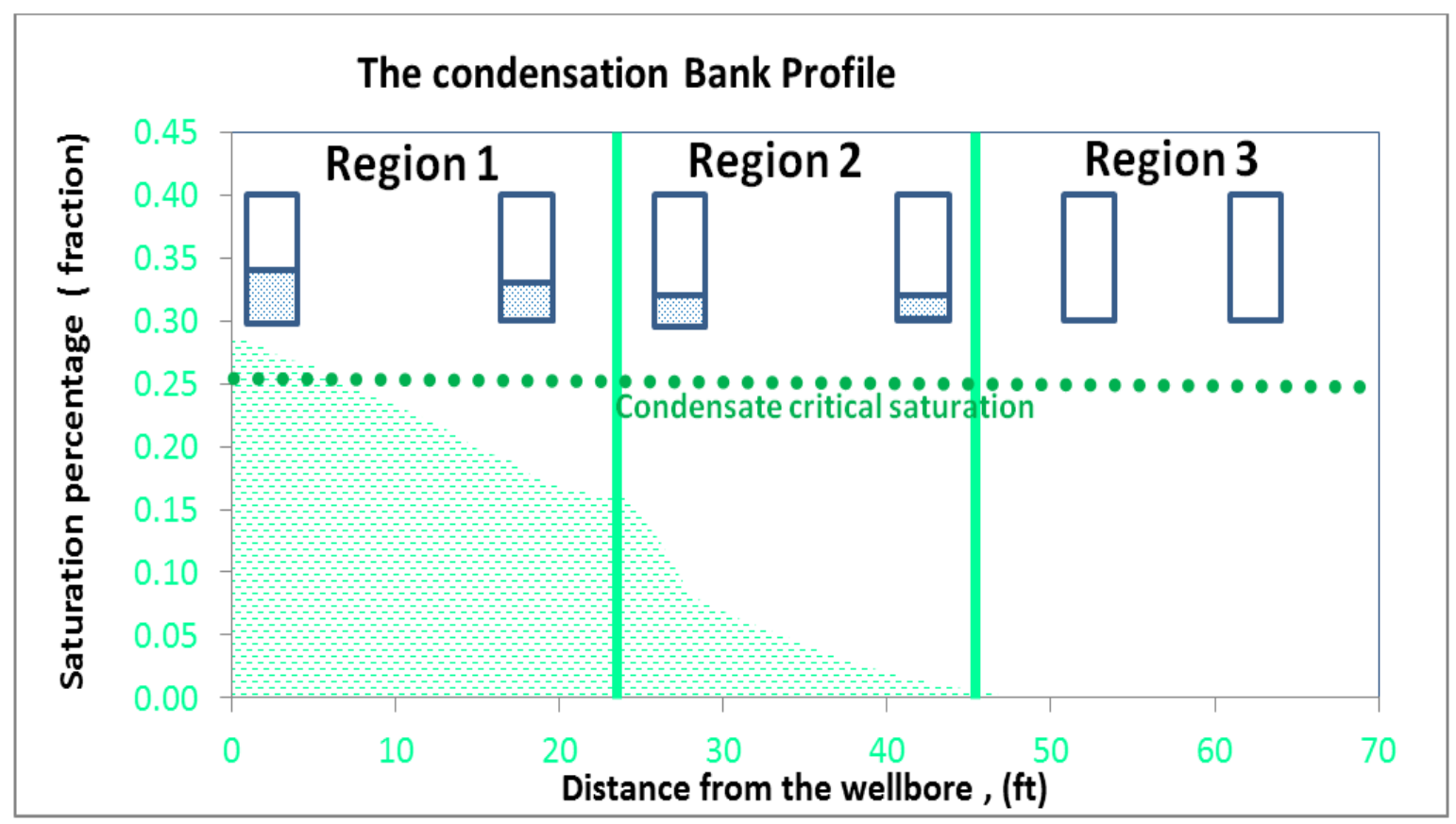

Figure 6: Schematic gas condensate flow spatial behavior in the reservoir 


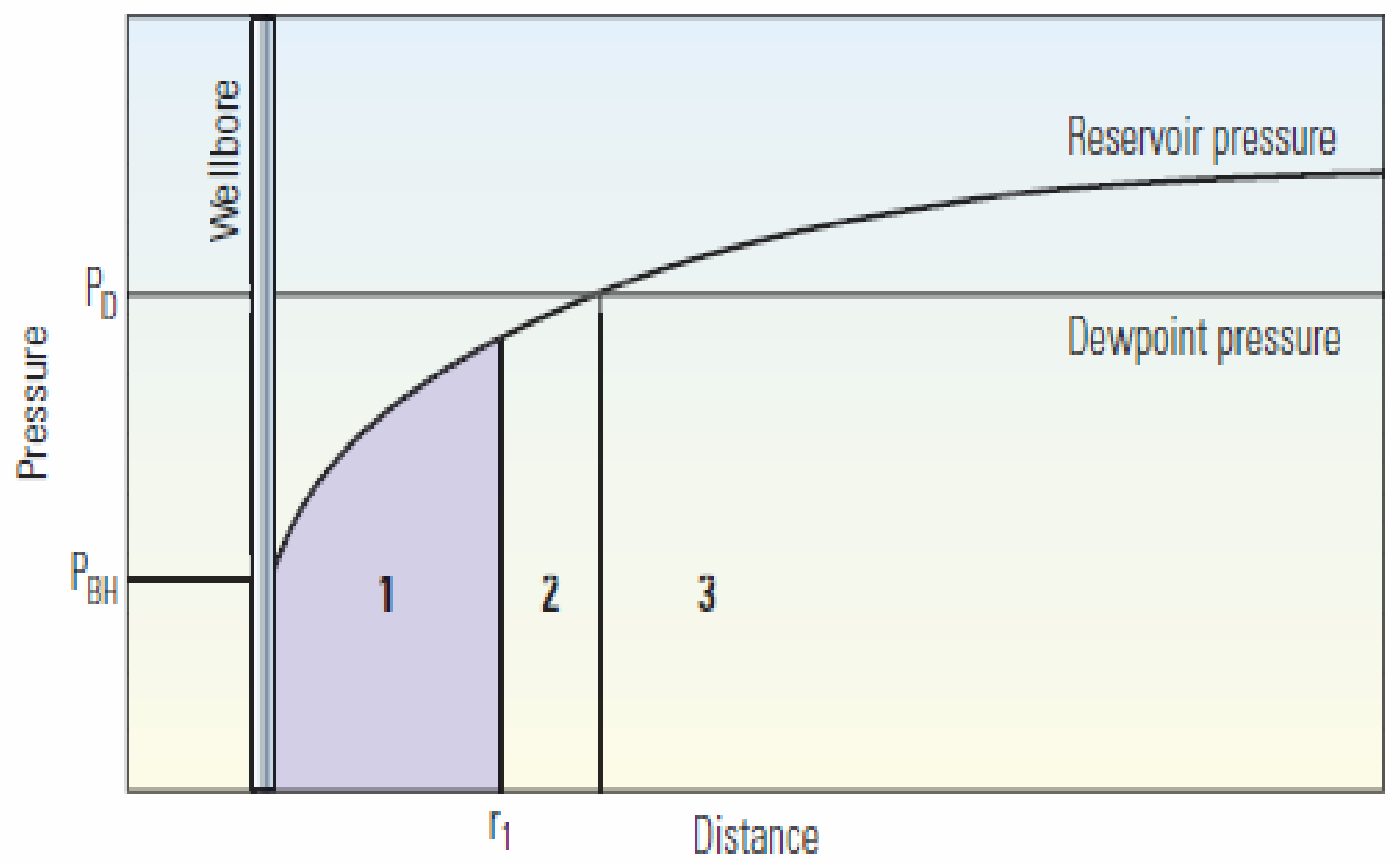

Figure 7: Schematic 3-region condensate bank development using the pressure profile [Fan Li et al. 2005/2006]

2.1.2.2. The Effect of Non-Darcy Flow on the Productivity Profile of Conventional Gas Reservoirs:

In conventional reservoirs the non-Darcy flow zone is significant around the wellbore due to high permeability and pressure gradient. This high velocity, low pressure zone forms a perfect spot for fluid to condensate and to accumulate around the wellbore. However, in reservoirs having a permeability greater than $250 \mathrm{md}$, phenomenon like non-Darcy effect was not noticed [Bourbiaux, 1994]. Figure 8 clearly shows productivity problems due to the non-Darcian effect in a conventional reservoir with different permeability. Reservoir fluid in this zone can be significantly affected by sharp loss in the heavy components. The impact of this phenomenon on condensate ring around the producing wells increases with the decrease of the reservoir permeability, therefore, the effect can be more severe in unconventional reservoirs with nano-permeability characteristics. We are going to discuss this issue in unconventional shale gas reservoirs section in more details later in this chapter. 


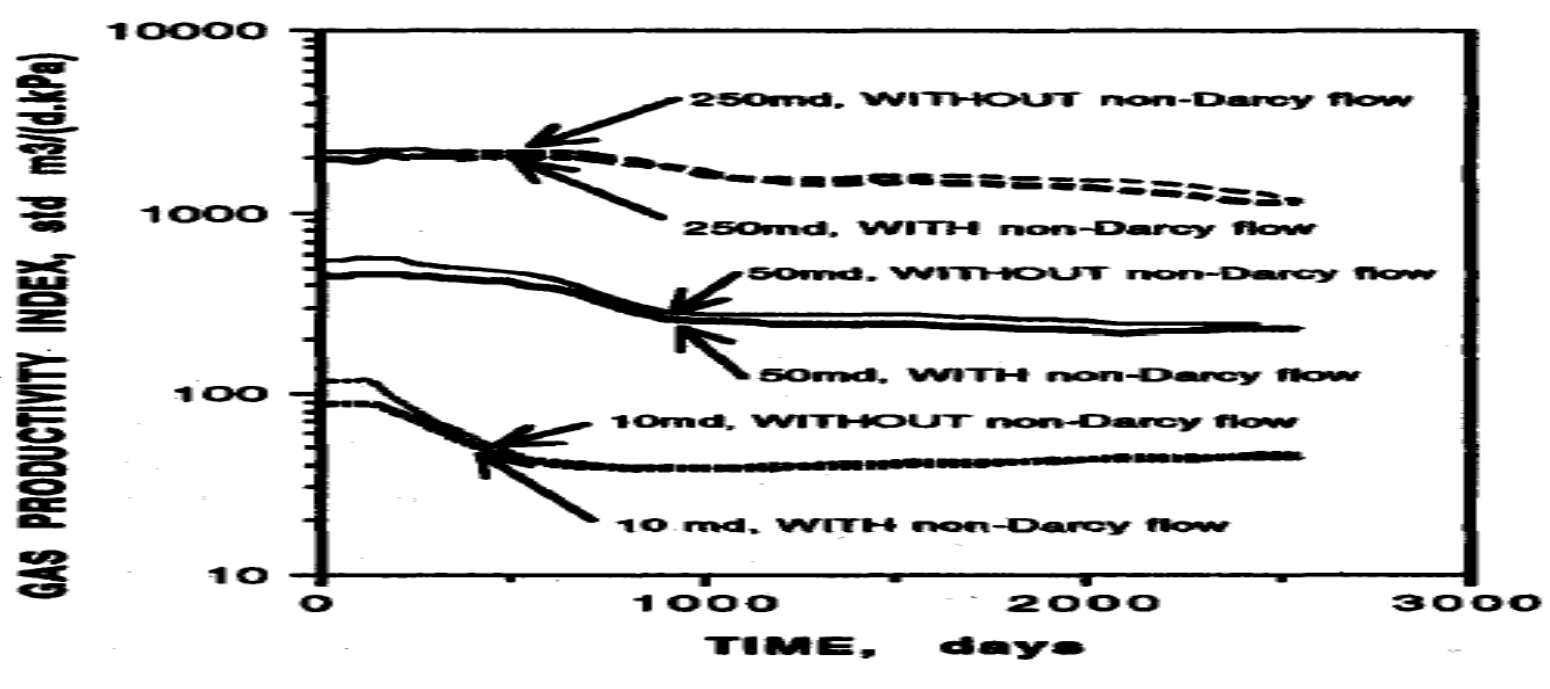

Figure 8: The productivity profile regarding the non-darcy flow [Bourbiaux,1994].

Due to the importance of this phenomenon, with decreasing trend of the absolute reservoir permeability, it was also reported that in low permeability reservoirs this has a great impact on the performance of hydraulically fractured wells even at low flow rates. Miskimins et al (2005) reported 5-30 \% gas flow capacity reductions in low rate flowing horizontal wells due to the non-Darcy effects by itself. Figure 9 shows the impact of nonDarcy flow on the productivity of a horizontal well with a fixed fracture length. However, with the optimized hydraulic fracturing technique this influence can be minimized, since the fracture length granted to reduce the accumulation of the condensate in the reservoir [Zhilin et al, 2007]. In this case, the non-Darcy effect might be of less importance.

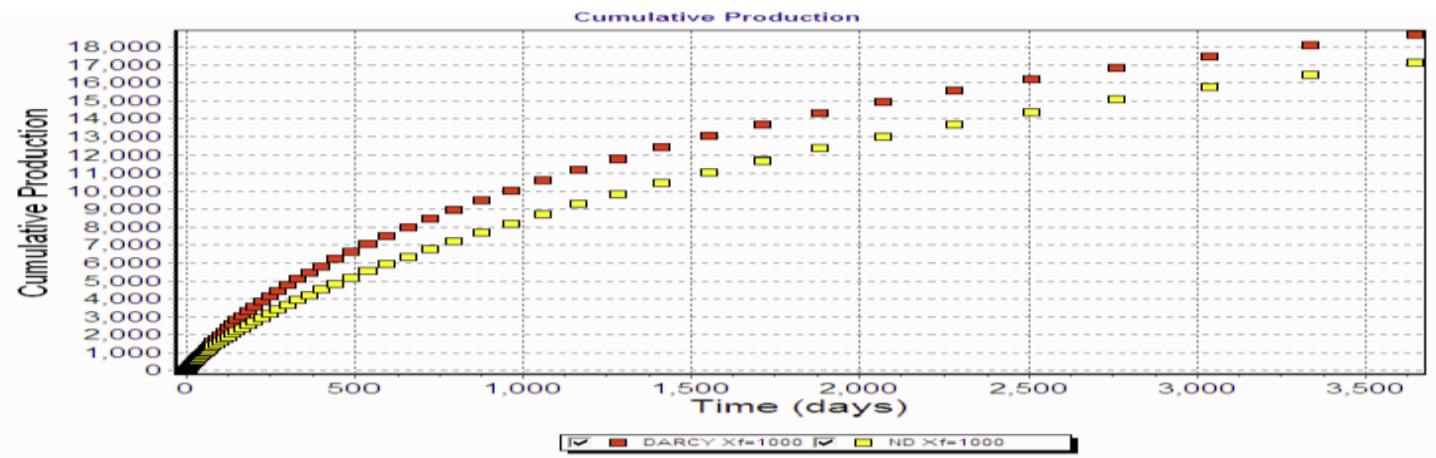

Figure 9: The effect of non-darcy flow on the cumulative gas production of a fractured well [ Miskimins et al. 2005] 


\subsubsection{Relative Permeability in Gas Condensate Systems:}

Since the condensate reservoirs are characterized by the coexisting phase behavior, a comprehensive awareness of the relation between those two phases has to be attained to thoroughly understand the condensate bank development. This can be accomplished by studying the mobility of the deposited condensate phase and the condensate build up mechanisms through the relative permeability of each flowing phase and the trapping number while the reservoir is being depleted. The relative permeability behavior has been demonstrated by researchers by means of the experimental modeling and analytical solutions. A simple model of the gas-condensate coexistence in the flow channels wetted by the liquid phase is illustrated in Figure 10. An inverse relationship can be noticed from the relative permeability profile between the relative permeability to the gas and the relative permeability to the condensation as the distance increases from the wellbore. The same scenario happens for the decrease in the condensation saturation.

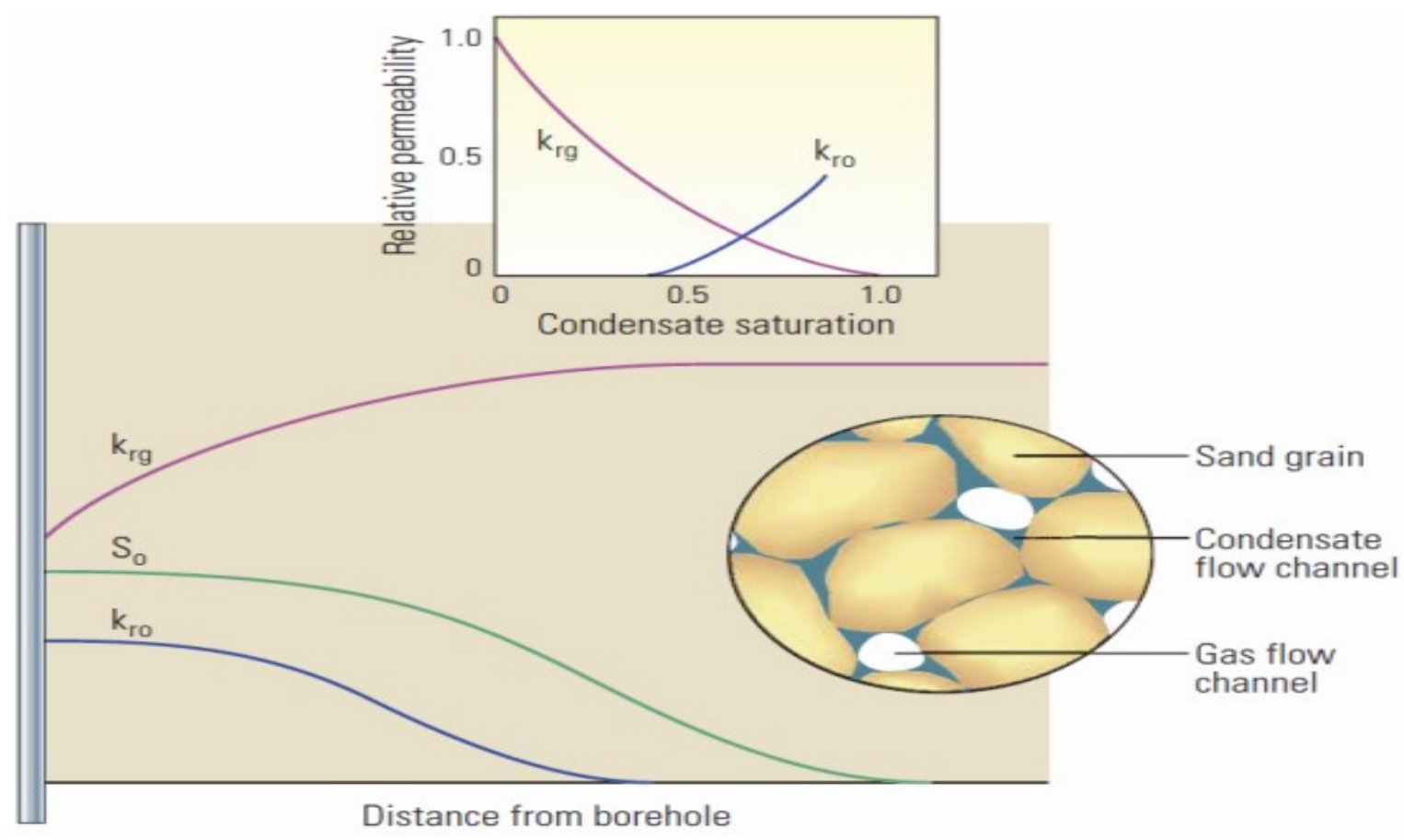

Figure 10: Typical relative permeability behavior in the conventional reservoirs [Fan Li et al., 2005/2006]. 
Since it is an active research area, besides the conventional methods in the oil industry to determine the relative permeability using Modified Brooks-Corey equations (MBC) and Carman-Kozeny formulation, experimental and modeling techniques have been developed and applied to improve our understanding of the fluid behavior under the reservoir condition. Even though it was believed that the relative permeability depends mainly on the pressure, the fluid nature and the local saturation [Pope, 1998], the experimental studies Curtis et al, (2010) and Mott et al (1999) proved that relative permeability of the gas is a function of $\left(\mathrm{K}_{\mathrm{rg}} / \mathrm{K}_{\mathrm{ro}}\right)$ ratio. However, since commonly liquid is the wetting phase, liquid is generally immobile due to the effect of the capillary forces. The microscopic liquid droplets are trapped in small pores or the pore throats where the capillary pressure may be substantially higher. This implemented that relative permeability is a dependent of the capillary number, which is basically the general form of collective effects of pressure gradient, the viscosity of the fluid and the capillary forces. This was expressed as:

$$
\mathrm{Nc}=\frac{V p g * \mu g}{\sigma g o}
$$

Where: $\bigvee_{p g}$ : gas pore velocity, $\mu_{g}:$ gas viscosity, and $\sigma_{g o}:$ interfacial tension (IFT).

Disregarding the shortcoming of the widely used formulations in the industry due to the weak performance with the pore distribution structure, recent publications reported the confident ability of a new modification of these equations to predict the shape of the relative permeability curves if adequate data is available [Behrenbruch, 2006]. However, these fundamental problems became even worse in the case of unconventional gas reservoirs due to more physical processes involved such as multi pore structure and physical adsorption effects which is a focus of our study. More details about these issues will be discussed in the following section. 


\section{2 : Unconventional Gas Condensate Reservoirs:}

In recent years, the industry has moved toward the exploration and exploitation of unconventional reservoirs to support the ever-increasing demand for energy. Unlike the wide range of research being devoted to gas condensation in conventional reservoirs, only a few exploratory studies on unconventional gas shale reservoirs are available. They are mostly focused on the wells deliverability problems caused by the condensate bank development and the role played by reservoir parameters, such as rock and fluid properties [Devegowda et.al, 2012, Orangi et. al, 2011 and Zarragoicechean et.al, 2004]. Besides the tight nature of the shales, they are also characterized by multi-scale pore structure, which often leads to a very complicated transport and storage of fluid in these tight formations. The shale matrix contains Kerogen, which is an organic nano-porous material that has been dispersed within the inorganic matrix of shales, as portrayed in Figure 11.Darcian flow is often related to the big pores and the natural fractures in the inorganic material and molecular phenomena (diffusion and nonlinear sorption) mostly in the Kerogen pores [Akkutlu et al, 2012]. In spite of the multi-scale fractures present in the shale matrix, horizontal wells and multi-stage hydraulic fracturing technology are essential for significant production from the gas shale reservoirs because of the extra connectivity provided inside the reservoir [Arukhe et. al, 2009, Cheng, 2012 ].

Besides fluid transport mechanisms in shale, the pore wall confinements and specific surface areas have a great impact on the phase behavior and interfacial dynamics of the flowing gases inside the nano-porous organic materials, which highly influences the fluid density, viscosity, formation volume factor, and ultimately fluid flow and transport in these tight formations [Campos et. al, 2009, Rahmani, 2012, and Sudhir et. al, 2009 ]. 


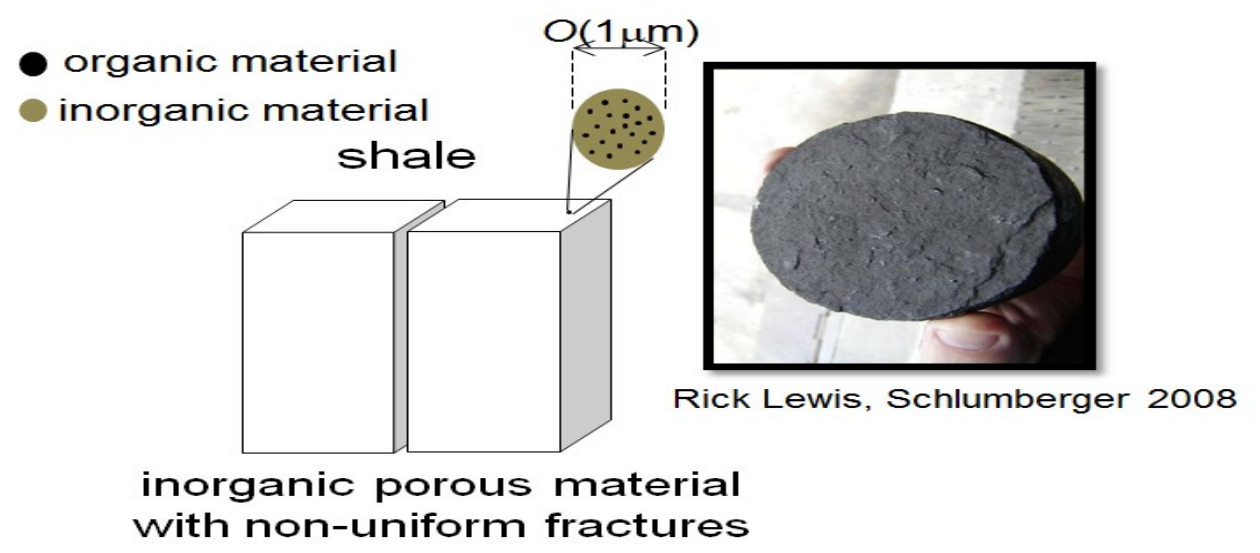

Figure 11: Distribution of the organic material inside the shale matrix [Kang et al, 2011].

\subsubsection{Imaging Techniques for Characterization of Shale Gas Reservoirs:}

There are different imaging techniques that have been introduced to the oil and gas industry, including Scanning Electron Microscopy (SEM), X-ray imaging, Transmission Electron Microscopy (TEM) or Scanning Acoustic Microscopy (SAM), that are able to better reveal the microstructure geometry, nature of the mineralogical components and the organics of complex formations like the gas shales. The visualization of shales using these techniques enabled the researchers to investigate the internal structure of such formations distinguished by the color variation. Curtis et. al (2010) has used the SEM to conduct a visual comparison illustrated in Figure 12 for nine different shale samples (the Barnett, Woodford, Eagle ford, Haynesville, Marcellus, Kimmeridge, Floyd, Fayetteville and Horn river) in terms of micro structure of the pores and composed material. In these images, the matrix is represented by the gray color: dark-gray regions are kerogen and light-gray regions are the inorganic constituents. The pores are shown in black. Clearly, these images show finely-dispersed porous kerogen pockets imbedded in an inorganic matrix. Moreover, the magnification of the SEM picture below in Figure 13 provides a typical Kerogen network of the pores and a close look at shale microstructures. At a fundamental- level, two completely different porous media, i.e. organic and inorganic, are revealed in organic-rich shale matrix. These observations 
are going to be important later when we will discuss the choices of path for condensate flow and transport in the shale matrix (organic/inorganic) pore system.

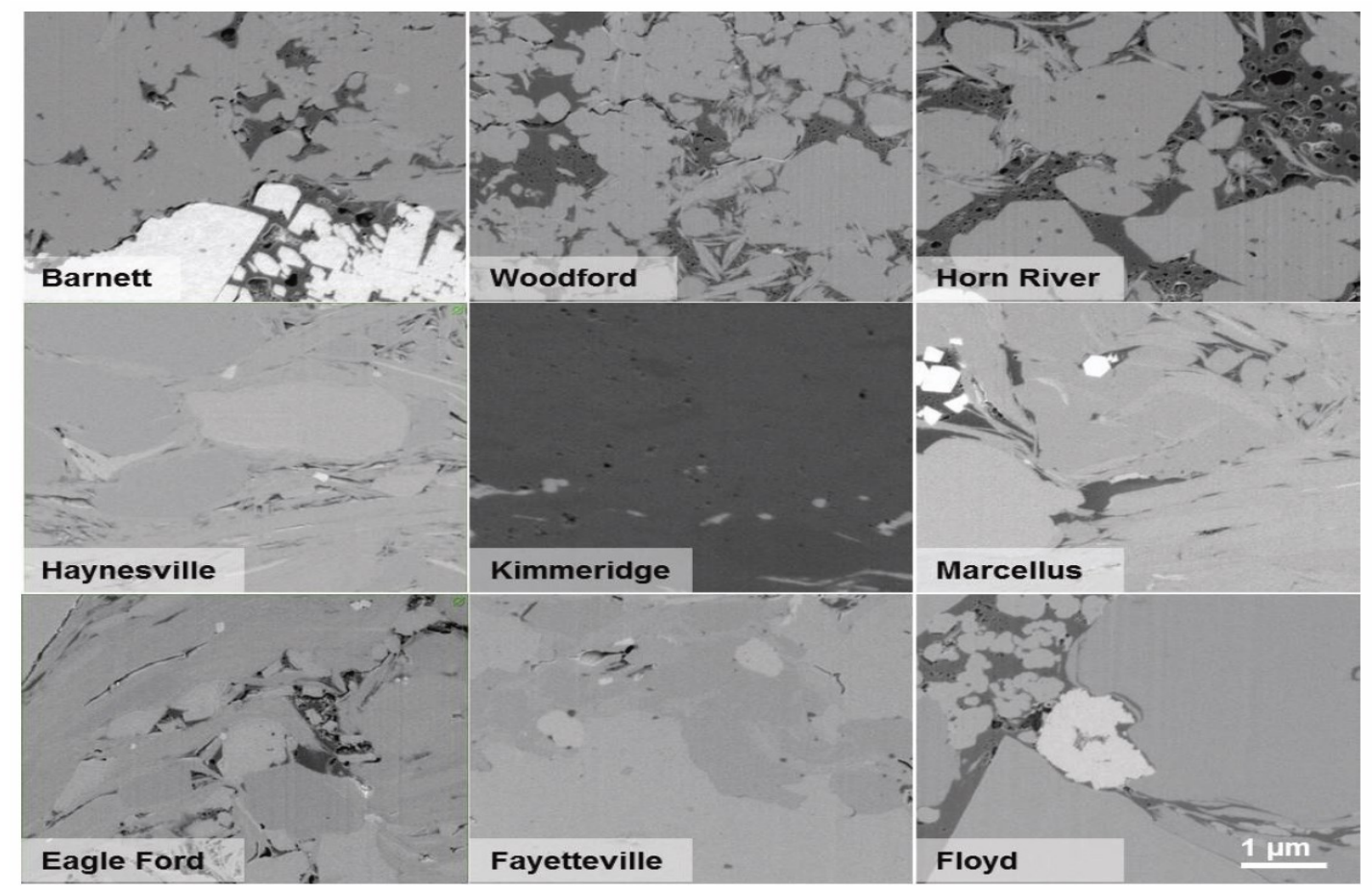

Figure 12: FIB/SEM picture shows cross section view of 9 shale samples adopted from Curtis et al. (2010). Dark grey areas are Kerogen and the bright grey is in organic material and the pores as black dots .

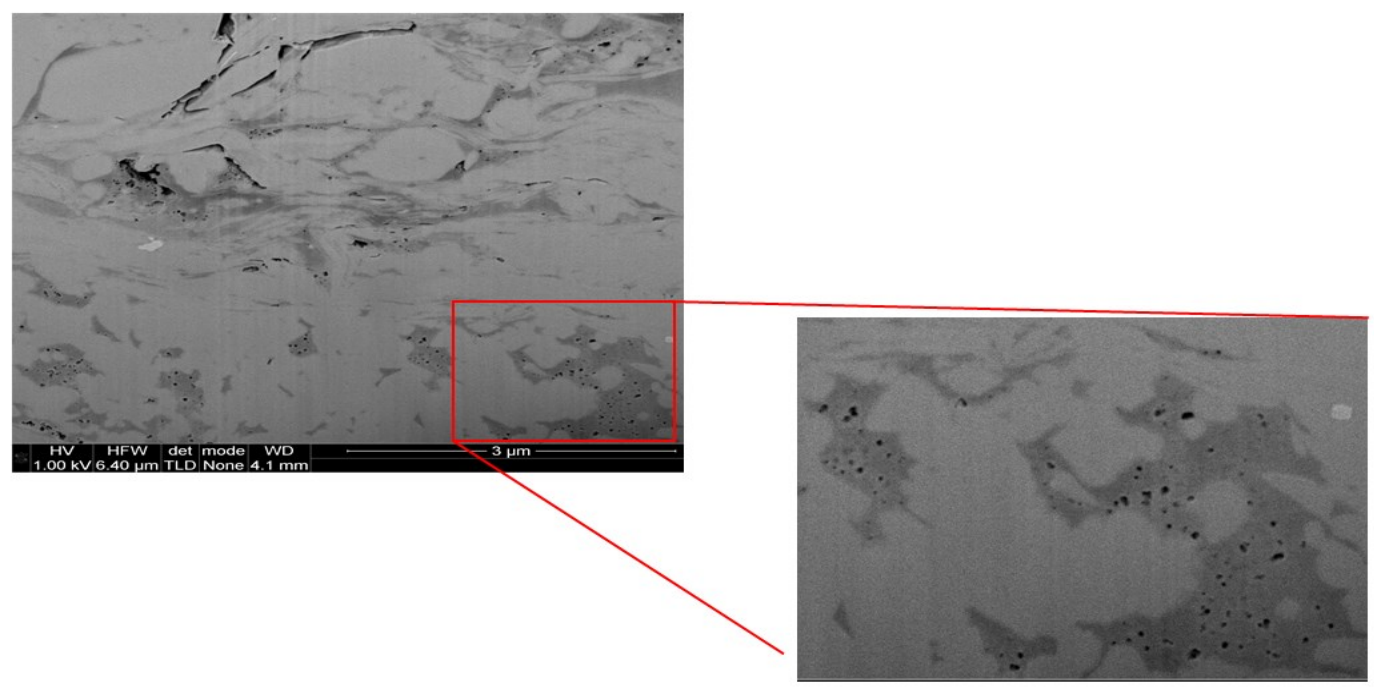

Figure 13: FIB/SEM image shows a scope on nano-pores within the organic material [Ambrose et al,2010] 


\subsubsection{Shale Matrix Gas Storage:}

Although it is widely known as an impermeable sedimentary rock with low porosity, shale has the ability to store significant amounts of gas because of its finely dispersed organic matter (i.e., kerogen), usually reported as Total Organic Content (TOC) as a weight percentage. The organics distribution, habit and concentration become important in any economic assessment. Recent work by Wang and Reed 2009; Moncrieff 2009; and Loucks et al. 2009 have documented the unique role of organics in the shale gas system. Gas shales unlike other lithologies contain significant quantities of organic matter in various stages of maturation. The organics add new dimensions to the shale, e.g. they lower density, increase porosity, provide the source of the gas, impart anisotropy, alter wettability and introduce adsorption [Sondergeld, 2010]. Figure 14 shows the porosity distribution within the shale matrix in Barnett formation. However, compaction on the other side is a common property in shales that has a concern impact on the shale storability because it reduces pore space and aligns platy minerals such as clays which results in highly anisotropic mechanical, elastic, and transport properties.

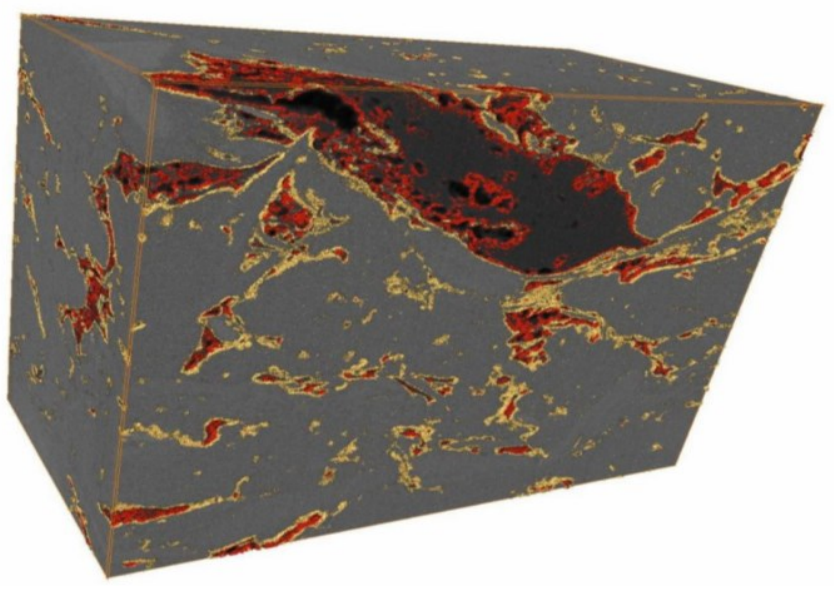

Figure 14: Most of porosity is related to the Kerogen. Yellow outlines are 3D Kerogen network and the red outlines are the porosity [ Ambrose, 2010].

The estimation of the original Hydrocarbon in place (OHIP) is one of the fundamental steps for the engineers to develop any reservoir that requires the best knowledge of the porosity and the permeability of the formation. Using the advanced 
imaging technique helped to clarify the uncertainty of the pore volume. From the SEM image shown in Figure 14, it can be noticed that the porosity distribution within the shale matrix is directly related to the large inter-connected pockets of the kerogen. Although the advanced technology succeeded so far to give a comprehensive view on the internal structure of the shale formations it is still unable to reach out the pores less than 4-5 nano meters. However from where the advanced imaging technique stopped, the pores which were contacted through an experiential approach, which was presented by Adelola et. al (2011) using Low Temperature Adsorption measurement on Barnett shale sample, showed a great interest in the nano scale pores. The results of her study, illustrated in Figure 15, revealed that the cumulative pore volume of pores up to $15 \mathrm{~nm}$ scale reached $0.0187 \mathrm{~cm}^{3} / \mathrm{g}$ that corresponded to $42 \%$ of the total core plug pores under the reservoir pressure [ Adelola et. al. 2011 ]. In few words, not considering the nano-pore size in the organic rich reservoirs can lead to miscalculating the engineering computation and under estimating the reservoir potential.

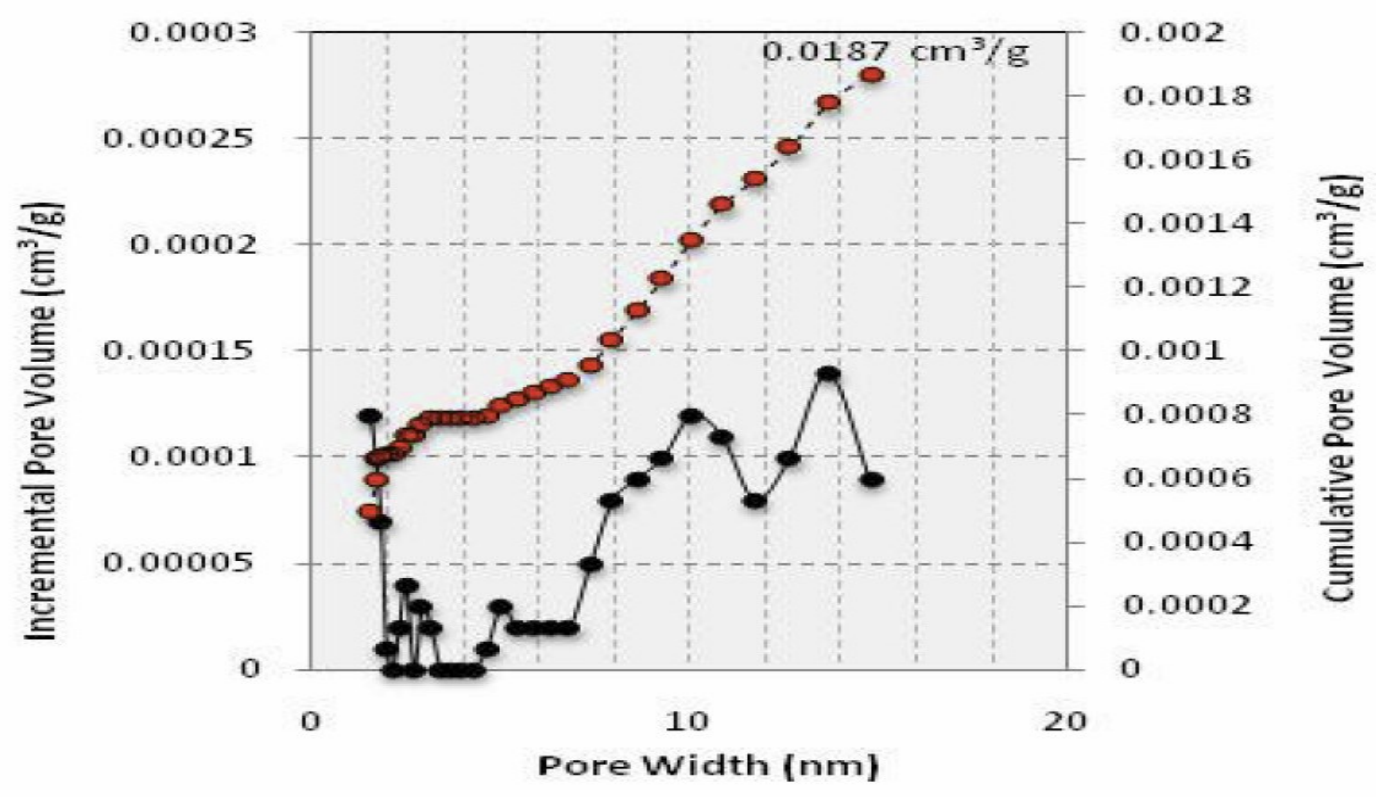

Figure 15 : Large portion of pore volumes are associated with Kerogen [SPE-147397]

In conclusion, the TOC and pore size distribution are critical factors controlling the shale matrix gas storage. The last is an indicator of the adsorption capacity of the organic pore walls to adsorb gas and develop a liquid like phase at the pore wall surface. 
The adsorb phase thickness, density and transport can influence the dynamics of condensate bank developments and saturation changes. The pore size distribution in those reservoirs also has a significant impact on condensate bank dynamics. It is well studied that as pore size shrinks to the size comparable to mean free path of the molecules, i.e. in the order of few nano-meters, fluid properties and phase behavior becomes not only a function of pressure, temperature and volume but also a function of pore diameter [Kang et al, 2011]. A significant amount of studies have been presented showing the importance of pore size on phase coexistence and fluid properties; however to our knowledge a widely appreciated equation of states does not exist that can fully describe this relation. Thus in this study we are attempting to come up with a general equation of state applicable for studying thermodynamics of fluids in shale gas reservoirs.

\subsubsection{Confinement Effects on Local Dynamics of Shale Gas Reservoirs:}

The effect of wall confinement on thermo-physical properties of fluids (i.e., $T_{c}, P_{c}$, $\mu_{\mathrm{f}}$ and $\mathrm{Z}$ - factor) in nano-scale-pores leads to deviation from their bulk values [Gelb et al,1999]. The porous media pore structure also has a significant impact on the fluid behavior. It is reported by Ortiz et al. (2005) using Monte Carlo simulation on the nanotubes that the critical temperature tends to decrease with pore size distribution. Rahmani,(2012) also stated that because of the adsorption phenomenon specially adsorbed phase density and adsorbed layer thickness effects in organic-rich-shales, the wall confinement effects is more significant in smaller pores than larger pores.

To fully understand the confinement effect on the fluid behavior in nano-pores, a significant amount of efforts have been devoted to overcome the difficulty of bringing experiment and theory together in a fruitful way. However, since these efforts were only consider the pore wall confinement effect and ignore adsorbed layer density and adsorbed layer thickness, they are still not good enough to apply in shale gas reservoirs therefore we are trying to overcome this problem by adding the missing physics. 


\subsubsection{SEMI- ANALYTICAL SOLUTIONS PROPOSED:}

A theoretical approach proposed by Zarragoicoechea and Kuz (2004) to estimate the deviation of critical temperature of pure gas components from their bulk value due to the pore confinement effects by the correlation presented in Equation (3) below. This approach demonstrated the proportionality between the specific temperature shifting and the relative size of the molecule to the pore size $\left(\frac{T_{c b}-T_{c p}^{*}}{T_{c b}} \alpha \frac{\sigma}{r_{p}}\right)$, where $T_{c b}$ and $T_{c p}^{*}$ are the fluid critical temperatures at the bulk and under pore confinement effect, and $\sigma$ and $r_{p}$ are critical molecule diameter and the pore radius. This relation was derived based on the generalized van der Waals EOS and the mean field model (Helmholtz free energy), i.e., presented in details in the appendix B1, equations (B.1.1-9), for Lennard Jones fluid model at which the pairwise potential resulting from the interaction of spherical particles is dependent on the distance between the pairs.. The results of this work were matched closely with the published experimental data (Morishige et al, 1997, and Morishige et.al, 1998) as shown in Figure 16. A similar relation was suggested in order to account for change in critical pressure and was applied to calculate the pressure shifting by the authors in equation (4); however it was not confirmed with experiment data. This approach also assumes neutral pore walls where the adsorption and desorption effect on fluid critical properties are ignored.

$$
\begin{aligned}
& \frac{T_{c b}{ }^{*}-T_{c p}{ }^{*}}{T_{c b}{ }^{*}}=0.9409\left(\frac{\sigma}{r_{p}}\right)-0.2415\left(\frac{\sigma}{r_{p}}\right)^{2} \\
& \frac{P_{c b}{ }^{*}-P_{c p}{ }^{*}}{P_{c b}{ }^{*}}=0.9409\left(\frac{\sigma}{r_{p}}\right)-0.2415\left(\frac{\sigma}{r_{p}}\right)^{2}
\end{aligned}
$$




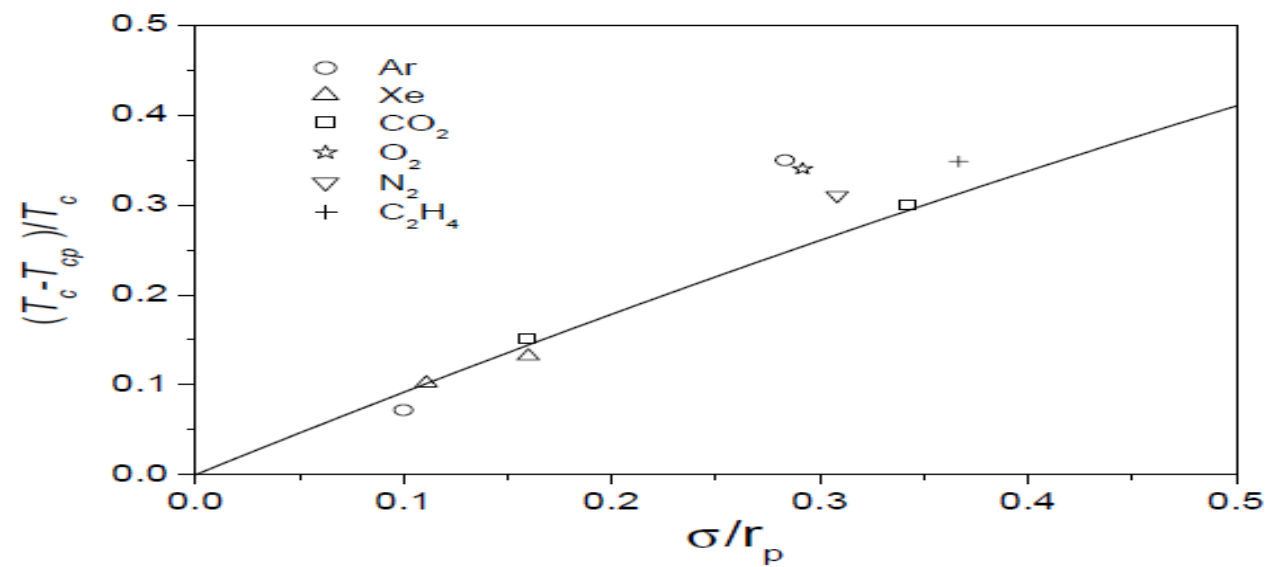

Figure 16: Matched the theoretical approach with experimental data

\section{Molecular Dynamics:}

The simulation of physical movements of the fluid atoms and molecules inside the pores can help in understanding of confinement effects on thermodynamics properties of the target reservoir fluid. This simulation basically include the description of the motion trajectories of atoms and molecules which are determined by numerically solving for the Newton's equations of motion for a system of interacting "N" number of molecules and atoms. Monte Carlo simulation is one of the widely used molecular dynamic methods to calculate the liquid gas coexistence envelope and observe the distinctive deviations in the critical properties for different hydrocarbon compounds bounded with very tight environment. [ Panagiotopoulos (1987); McDonald (1972); Rowley et al (1975); Kim et al (2003); Singh et al. (2009); Rahmani, (2012) ]. In these studies the investigation was mainly focused on the behavior of methane, $n$-butane and n-octane inside nano-scale pore systems with diameters between 0.8 to $5 \mathrm{~nm}$ and the products were validated with the experimental data Jiang et al. (2005). The agreement was good as it can be seen in Figure 17. Furthermore, since the aim was to apply the fact that shale gas systems may be characterized by organic and inorganic pore systems, they suggested that the deviations of critical properties for those hydrocarbons to be different as the pore surfaces become different (mica and graphite) as it is shown in Figure 18. 
Following these studies an exponential decrease in the critical temperature with the reduction of the pore size was observed in Panagiotopoulos' work (1999) who suggested that at the extreme hypothetical value of a zero pore diameter, the $T_{c}$ limits to zero. The Singh (2009) also reported a similar exponential trend for the critical temperature and pressure deviation with the pore size using the molecular dynamic study illustrated in Figure 17; the results of his work was shown in Figure 18.

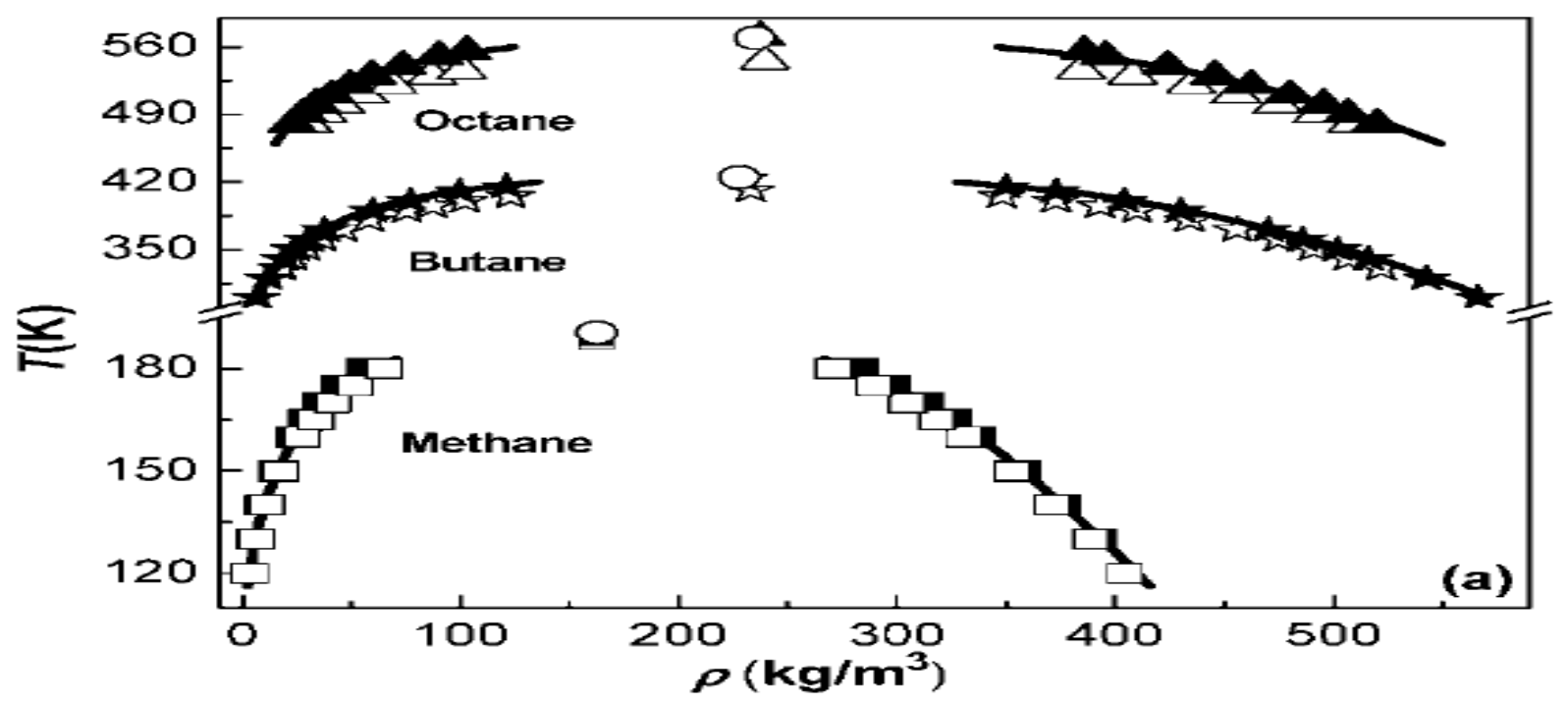

Figure 17: Molecular dynamics results (the empty shapes) matching with experimental data (the bolded shapes).[Singh, 2009]
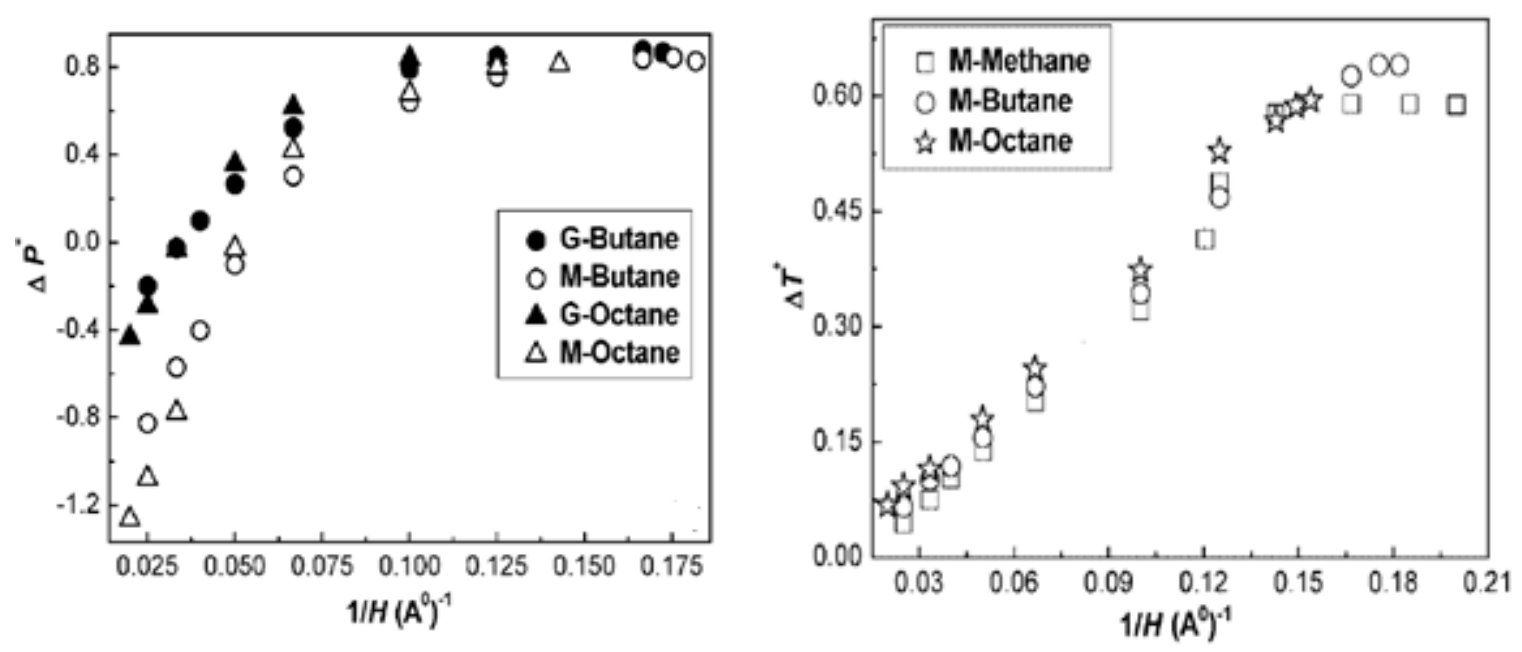

Figure 18: The deviation in the critical properties of pure n-alkanes components Vs. the inverse slit width $(H)$ in Graphite and Mica pores [Singh 2009]. 
Singh's work has been extended by others to various pore sizes and different components by applying a curve fitting and extrapolation of the temperature and pressure deviation established for three components Methane, Butane and Octane. Sapmanee, (2011) used this curve fitting method for three points (pure gases) used by Singh to predict the behavior of gas mixtures in a range of organic (graphite) pore system to resemble the shale gas reservoir condition as it is demonstrated in Figure 19 for pressure and temperature variations.
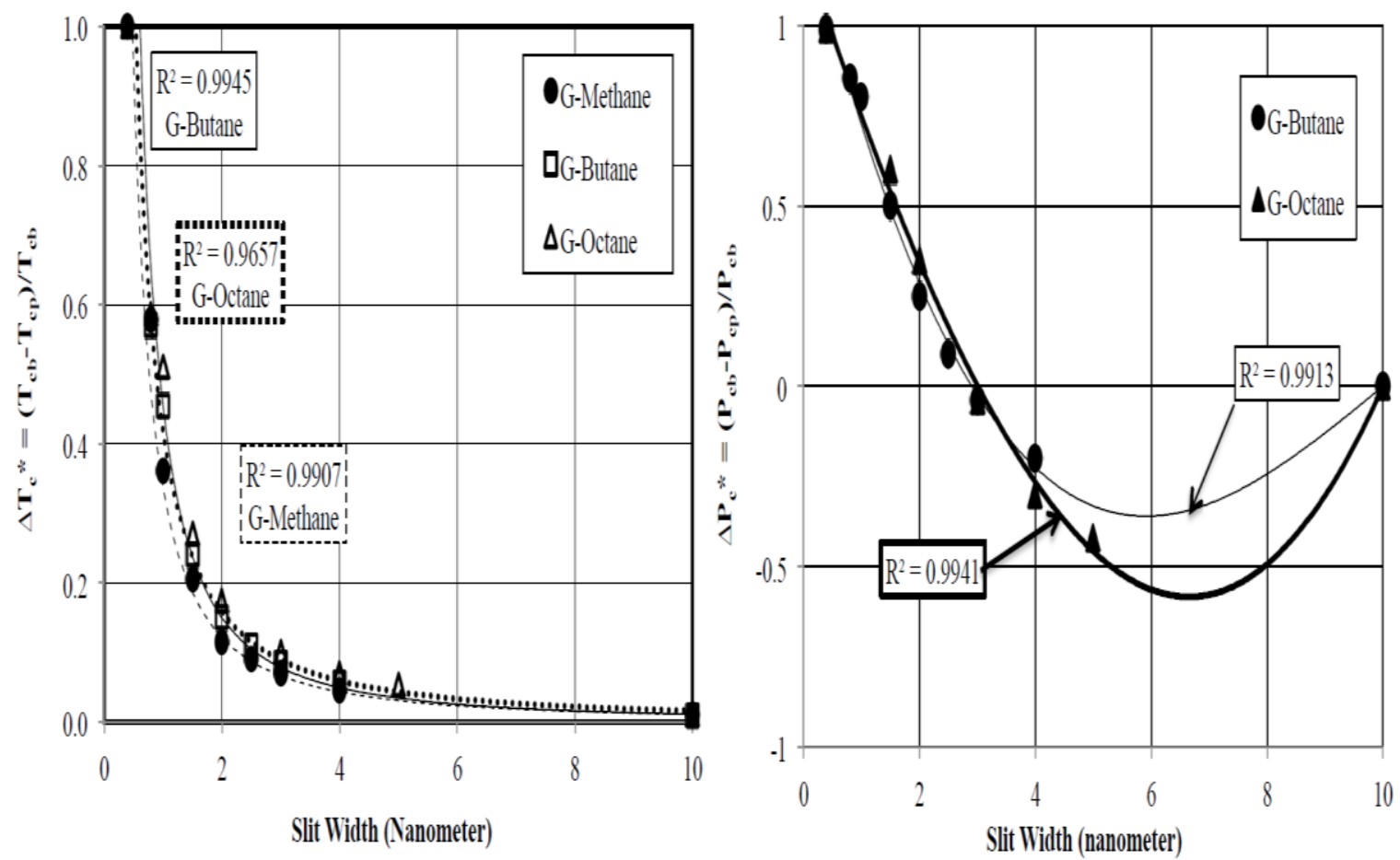

Figure 19 : Curve fitting method to reach out various organic pore sizes adopted from Sapmanee (2011).

The second step in their approach was the application of these correlations to other hydrocarbon compounds using extrapolation. The graph, Figure 20 shows the extrapolation scheme, was constructed using the critical shifting values of methane, Butane, and Octane at different pore sizes obtained from the curve fitting method used above and their corresponding molecular weights. However they did not provide the rationale behind their extrapolation approach and justification of this approach has not been presented by any experimental or theoretical approach. 

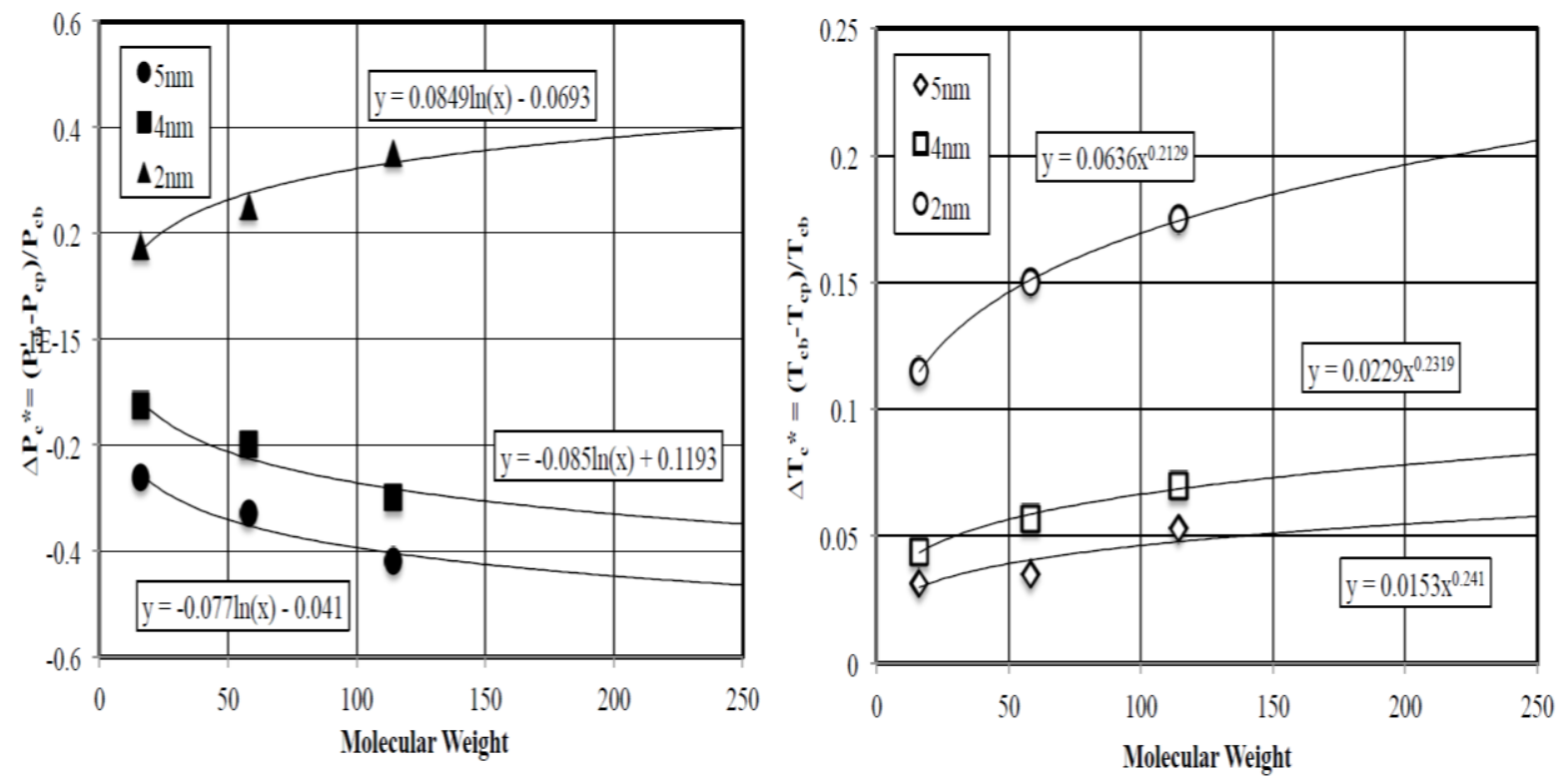

Figure 20 : Extrapolation for range of components adopted from Sapmanee (2011)

\subsubsection{Adsorption Effect on the Gas Flow inside Nano-Porous Media}

Gas molecules inside organic nano-pores have limited kinetic energy due to the wall confinement and gas adsorption effects. This in turn affects the critical properties of the gas mixture leading to change in the phase envelop of gas mixture. Therefore conventional PVT analysis might not lead to realistic description of fluid thermodynamics in these organic nano-pores. Akkutlu (2012) described the thermodynamic equilibrium inside the organic pores, as shown in Figure 21, suggesting that the lessened activity of the gas molecules closer to the pore walls is because of the stronger influence of pore wall confinement. These molecules participate in the formation of a dense (liquid-like) adsorption layer that covers internal surfaces of the pore walls. Next to the adsorption layer is the phase-transition layers where the molecules are constantly being adsorbed and desorbed under equilibrium. The molecules in phase transition are relatively less dense and more mobile with some kinetic energy, although they are under somewhat reduced influence of the walls. The rest of the gas molecules are at the central portion of the pore. Adsorbed phase density is a very important quantity that cannot be easily determined using an experimental approach and requires detailed analytical and numerical considerations that will be discussed in the next chapter. 


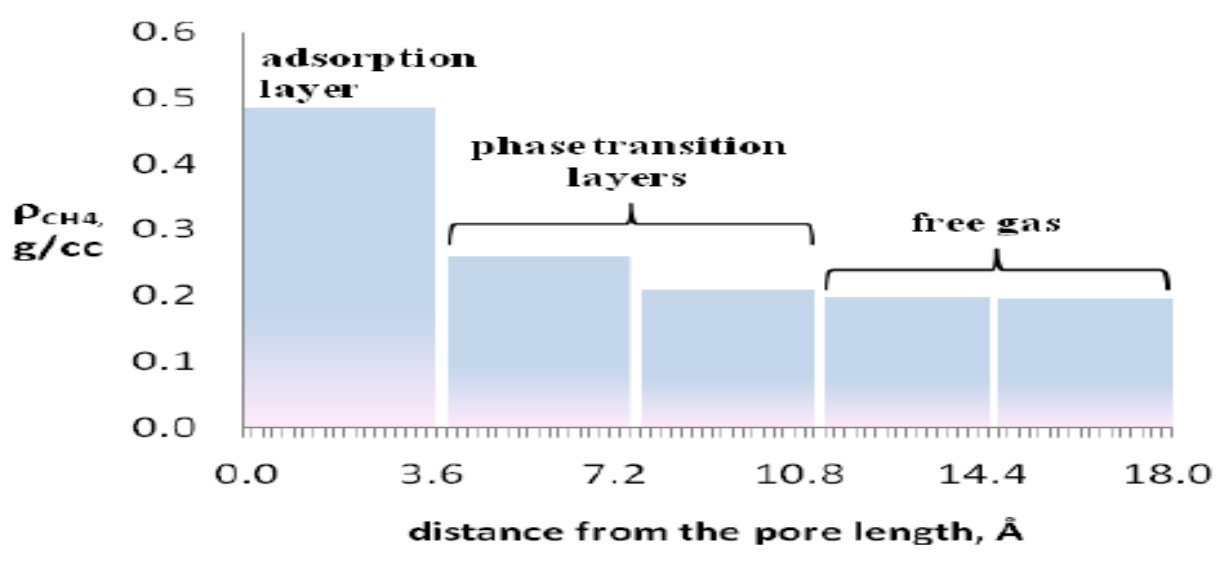

Figure 21 : Simulation of gas flow inside the pores network (Molecular layer density for methane at $176^{\circ} \mathrm{F}$ $\left(80^{\circ} \mathrm{C}\right)$ across the half-length of a 3.74-nm organic slit pore.). Adopted from (SPE-134583)

\section{CHAPTER: 3: METHODOLOGY}

\section{1: EOS Development:}

In physics and thermodynamics, equation of state (EOS), a mathematical relation between temperature, pressure, volume or internal energy, is widely used to describe the properties of fluids, fluid mixture phase equilibrium, and phase transitions. Different equations of state have been developed to account for real gas behavior, including Van der Waal's (VdW-EOS), Redlich-Kwong (RK-EOS), Soave-Redlich-Kwong (SRK-EOS), and Peng-Robinson (PR-EOS). In comparison to the ideal gas equation of state, these equations introduce two constants to account for pressure drop due to molecular attraction forces and volume occupied by the molecules [McCain, 1990]. Later SRK-EOS and PR-EOS introduced an additional parameter (i.e., the acentric factor) to give an ability to simulate different fluid types [Yuan et al, 2006]. Unfortunately, the applications of these equations are limited in shale gas reservoirs due to the presence of new physics that have not been considered while developing these equations; that is pore size distribution and adsorbed phase density. To account for these new physics, the generalization (VdW-EOS) is presented for a confined fluid in the nano-pore. This section will review the foundation of the adsorbed phase density of the fluid in organic porous media. Van der Waals equation of state in its general form can be expressed as: 
$\left(P+\frac{a}{V^{2}}\right)(V-b)=R T$

Where; $\mathrm{P}$ and $\mathrm{V}$ are the pore pressure and the molar volume respectively, and the "a" and " $b$ " are the corrections due to the internal energy and the molar volume respectively. The later constants can be calculated from critical properties of the fluid as following:

$$
\begin{aligned}
a & =\frac{27(R T c)^{2}}{64 P c} \\
b & =\frac{R T c}{8 P c}
\end{aligned}
$$

To account for the amount of gas being adsorbed, Rahmani, (2012) developed a theoretical method to estimate the adsorbed phase density using Van der Waals CoVolume Constant based on Dubinin (1960).

First it was suggested that if " $W$ " is the volume taken up by the adsorbed phase and $\alpha$ is the amount adsorbed, then,

$W=\alpha \boldsymbol{b}$

This can be expressed as $\frac{\alpha}{W}=\frac{1}{b}$

Then the equality can be weighted based on the molecular weight $(M)$ that leads to:

$$
\frac{M_{W} \alpha}{W}=\frac{M}{b}=\rho_{s}
$$

So, with known molecular weight and calculated co-volume constant at the critical temperature, the adsorbed phase density $\left(\rho_{s}\right)$ for a pure substance can be calculated from the equation above. 


\section{2: Tuning PR-EOS in Composition Simulator:}

In general, there is a good understanding of application of different equations of state to investigate the phase transitions in bulk fluids where the system size is not important. However, as the volume of the system shrinks to the meso- and micro-scales, the phase equilibriums become size dependent, where the wall confinement effects change the thermodynamic properties of the fluids significantly [Guillermo et al. 2002]. Experimental and numerical investigations on equilibrium and non-equilibrium thermodynamical properties of fluids in nano-porous materials show dramatic deviations from their bulk values obtained using PVT-cell measurements [Guillermo et al. 2002]. Recent studies show that as pore size decreases to the micro-scale, critical temperature, freezing, and melting points decrease. It is also observed that water viscosity reduces and critical pressure and interfacial tensions change significantly when the pore size decreases [Devegowda et al 2012, Sudhir et al 2009]. Campos showed through the use of molecular modeling and simulations that gas adsorption and solubility in liquid are enhanced due to dominant pore wall effects (2010). Following that, Rahmani (2012) investigated the effect of pore confinements on single and multi-component shale gas thermodynamics, and found that critical properties and fluid behavior confined in small micro-pores are significantly different than their bulk values. These observations imply the importance of further studies to develop a new equation of state that can accurately describe the phase transitions and interfacial dynamics under micro-scale confinements. Adsorbed phase density $\left(\rho_{\mathrm{s}}\right)$ in the organic pores can be calculated as discussed earlier. Our approach here is to extend Zarragoechea \& Kuz's equation that measures the change in the critical temperature of confined fluid in terms of the dimensionless ratio of the molecular and pore diameter $\left(\frac{\sigma}{r_{p}}\right)$, then include the adsorption layer density effect. Our theoretical approach will also be valid to describe the change in critical pressure that is different than what Zarragoechea \& Kuz suggested (2004).

According to the general gas law that describes the relationship among pressure, volume, and temperature by the formula $\left(\frac{P V}{T}=\right.$ Constant $)$, and by substituting the volume by the density of the unit mass, an inverse proportionality between the density of 
the gas and its temperature can be established. This is generally expressed as the following: ( $\rho \propto \alpha \frac{1}{T}$ ). However, inside the organic pores, since the sorption takes place, the calculation of gas density should include the adsorbed layer density. Assuming that the adsorbed phase density has a constant value that is not affected by the pore wall confinement, we proposed the change in the gas density inside the nano-pores by the empirical term $\left(\frac{\rho}{\rho_{s}-\rho}\right)$. This is denoted by $\rho_{\text {corr }}$ in later equations. Thus, we applied this correction to account for the adsorbed gas density inside the organic pores to equation 3 suggested earlier by Zarragoechea \& Kuz, 2004 to generate equation 14 :

$\rho \alpha\left(\frac{1}{T}\right)$

$\rho=\operatorname{Cons} *\left(\frac{1}{\mathrm{~T}}\right)$

$\mathbf{T}=\left(\frac{1}{\rho}\right) *$ Cons

$\frac{T_{c b}{ }^{*}-T_{c p}{ }^{*}}{T_{c b}{ }^{*}}=\frac{0.9409}{\rho_{\text {Corr }}}\left(\frac{\sigma}{r_{p}}\right)-\frac{0.2415}{\rho_{\text {Corr }}}\left(\frac{\sigma}{r_{p}}\right)^{2}$

The same procedure was followed to establish the density-pressure relations, which has different proportionality from the temperature. The correction was applied to equation 4 to generate equation 18 :

$$
\begin{aligned}
& \begin{array}{lll}
P & \alpha & \rho
\end{array} \\
& \mathbf{P}=\text { Cons } *(\boldsymbol{\rho}) \\
& \mathbf{P}=\left(\boldsymbol{\rho}_{\text {corr }}\right) * \text { Cons }
\end{aligned}
$$

Where $\rho_{s}$ is the adsorbed phase density 
Since no molecular dynamics simulation was carried out in this thesis, adsorbed density is calculated using equation (7) and (10). In these equations bulk values of critical pressure and temperature $\left(P_{c}\right.$ and $\left.T_{c}\right)$ have been used and adsorbed phase density for Methane and Butane is calculated, i.e., 0.372 and $0.499 \mathrm{~g} / \mathrm{cc}$ respectively.

The evaluation of this correction was processed though a comparison of critical temperatures based on different pore size obtained using between, the results from Zarragoechea \& Kuz's model, the molecular dynamic solution reported by Singh et al, 2009 used as a reference case, and the corrected semi-analytical solution. Figure 22 and Figure 23 show the validation results for methane and Butane respectively.

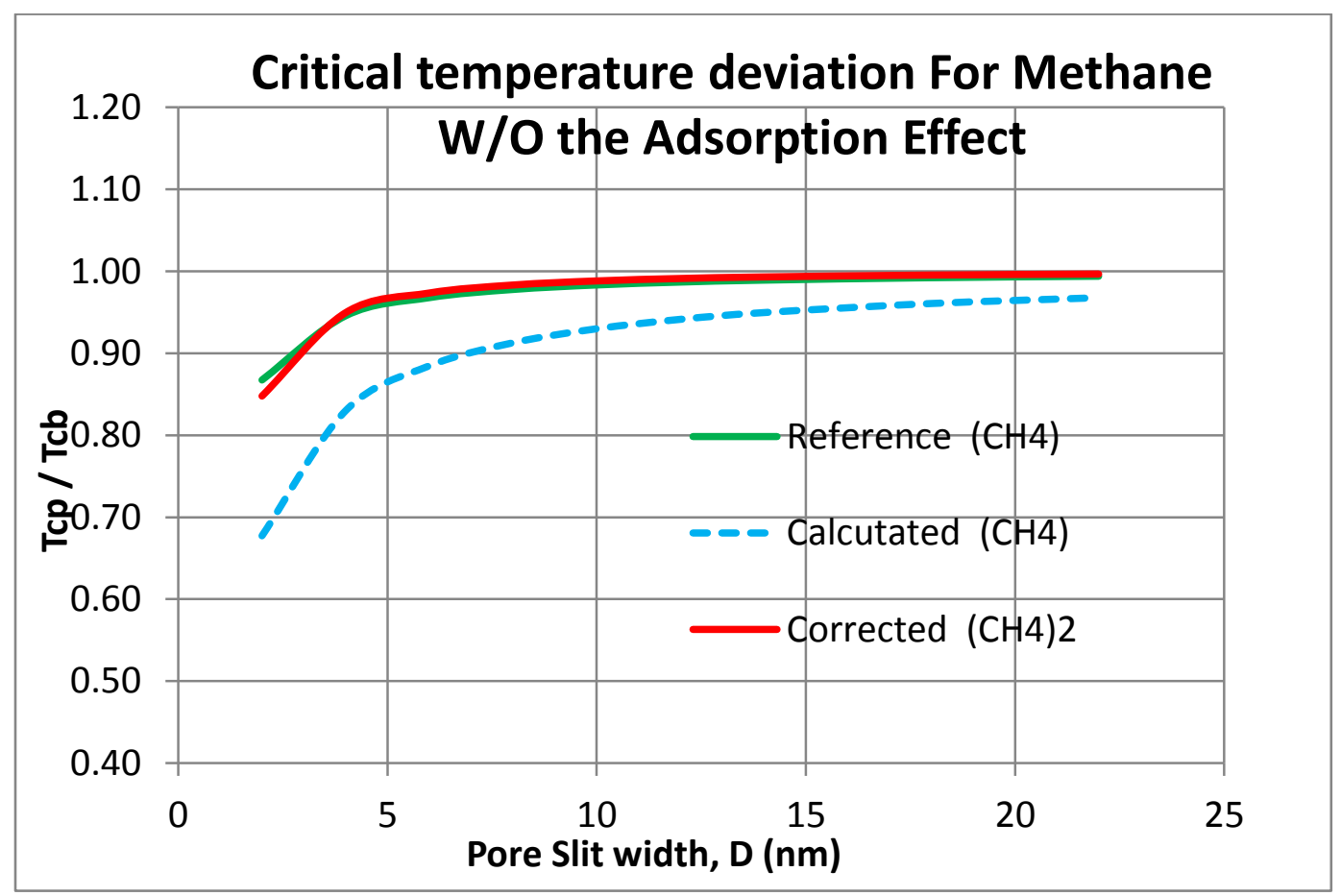

Figure 22: Correlation shows the outcome difference between a semi-analytical solution (dashed blue line), molecular dynamic solution (solid green line), by Singh et al. (2009) used as a reference, and the correction approach (solid red line) in graphs for Methane using different pore diameters. 


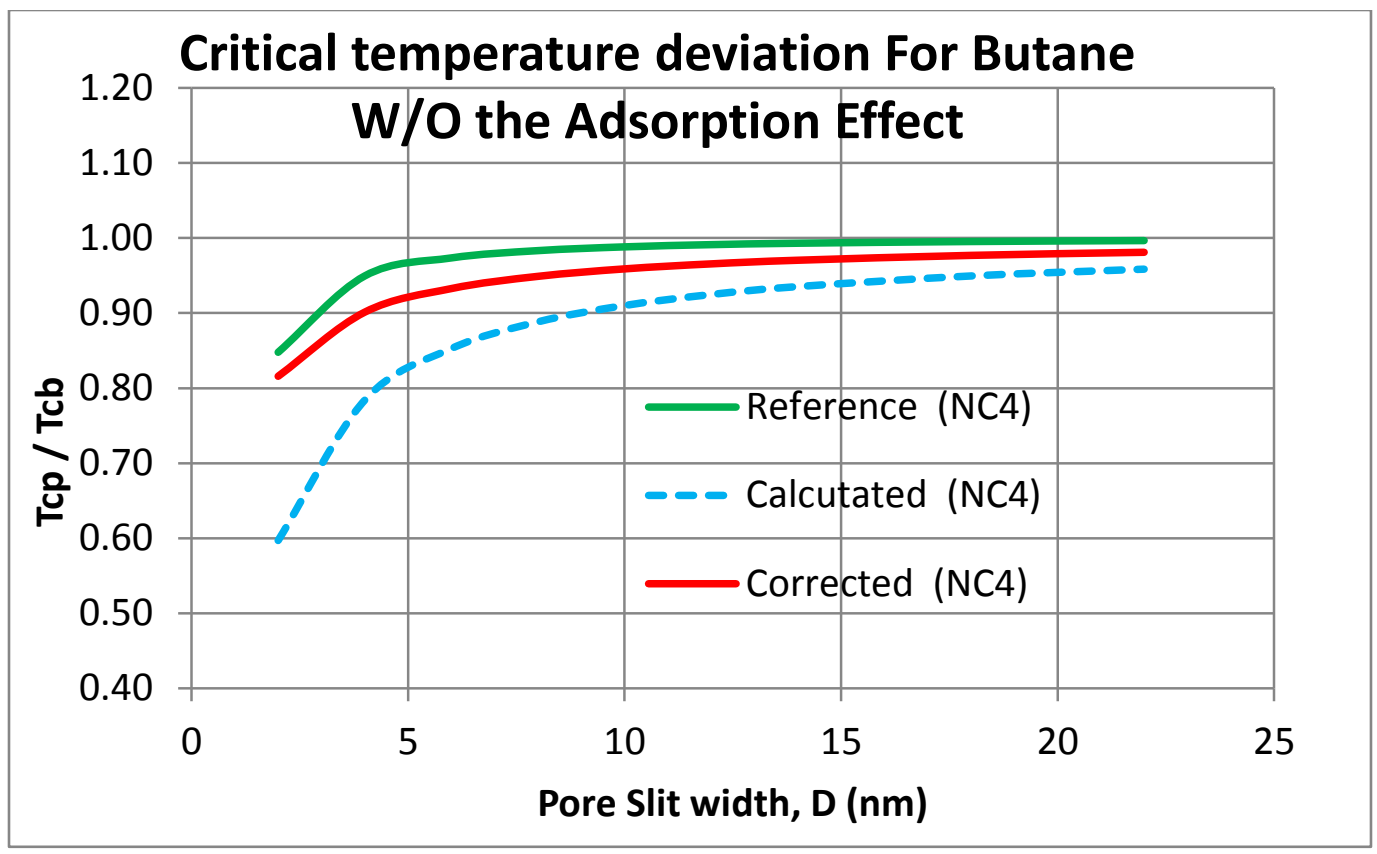

Figure 23 : Correlation shows the outcome difference between a semi-analytical solution (dashed blue line), molecular dynamic solution (solid green line), by Singh et al. (2009) used as a reference, and the correction approach (solid red line) in graphs for Butane using different pore diameters.

The match between the corrected semi-analytical solution and the molecular dynamics results by Singh et al (2009) was quite good as it is shown in two figures above. Moreover, as it is well known that all paraffin hydrocarbon components belong to same homologous series with graded properties. This gave us the confidence to appropriately apply this correction to different paraffin components of fluid mixture. Figure 24 shows the critical temperature deviation and Figure 25 illustrates the critical pressure deviation.

Table 2 shows details of the compositions used as the base case fluid model in this work [Imo-Jack, 2010]. Although the shifting of the critical temperature of pure substances due to pore wall confinement is reported earlier in literature, in this work we extended that to organic nano-porous materials such as shale. Moreover, our approach released the confusion related having the negative values of the pressure in correlations seen in the literature (Sapmanee, 2011) using old technique. In conclusion we are using these new correlations to correct dritical properties of pure paraffin components and use that in calculations of the phase envelope for the gas mixture used as a fluid model in 
this study. These corrected values are then used to calculate the z-factor, the gas density, and the co-volume coefficient (b) in PR-EOS equation using the integrated equations suggested by the Winprop application in CMG Ver.2012. And finally, all these calculations were deployed to generate the right envelope for the gas mixture.

\begin{tabular}{|c|c|c|c|c|}
\hline Component & PC (atm) & TC (K) & Acentric fact & Mol. Weight \\
\hline \hline CO2 & 72.8 & 304.2 & 0.225 & 44.0 \\
\hline N2 & 33.5 & 126.2 & 0.040 & 28.0 \\
\hline CH4 & 45.4 & 190.6 & 0.008 & 16.0 \\
\hline C3H8 & 41.9 & 369.8 & 0.152 & 30.1 \\
\hline IC4 & 36.0 & 408.1 & 0.176 & 44.1 \\
\hline NC4 & 37.5 & 425.2 & 0.193 & 58.1 \\
\hline NC5 & 33.4 & 460.4 & 0.227 & 72.2 \\
\hline NC5 & 33.3 & 469.6 & 0.251 & 72.2 \\
\hline NC7 & 29.3 & 507.4 & 0.296 & 86.2 \\
\hline
\end{tabular}

Table 2: Base case fluid model with physical properties (Win Pop 2012)

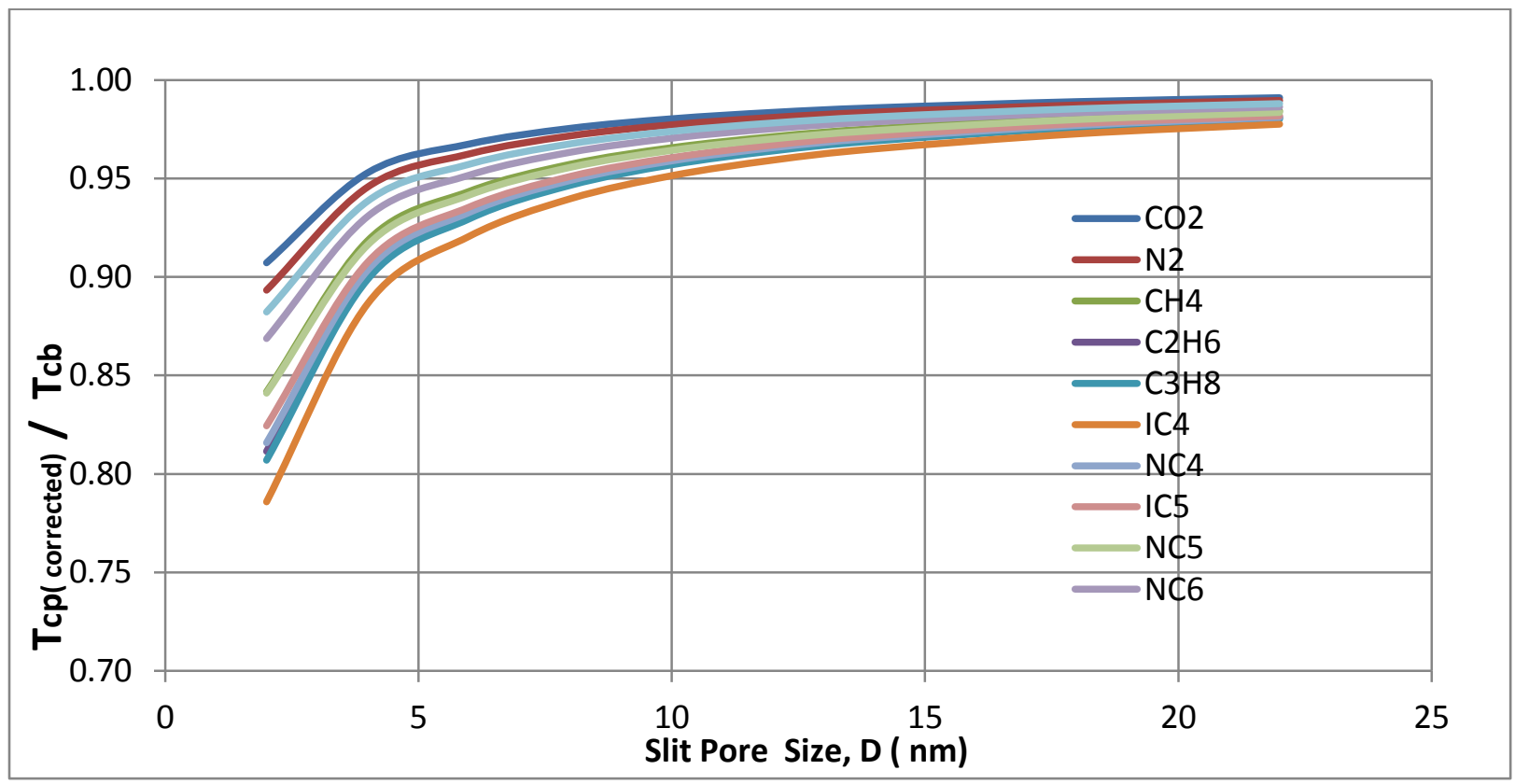

Figure 24 : The percentage of critical temperature deviation from the bulk value deviation for pure components using the corrected semi-analytical solution at a range of pore sizes 


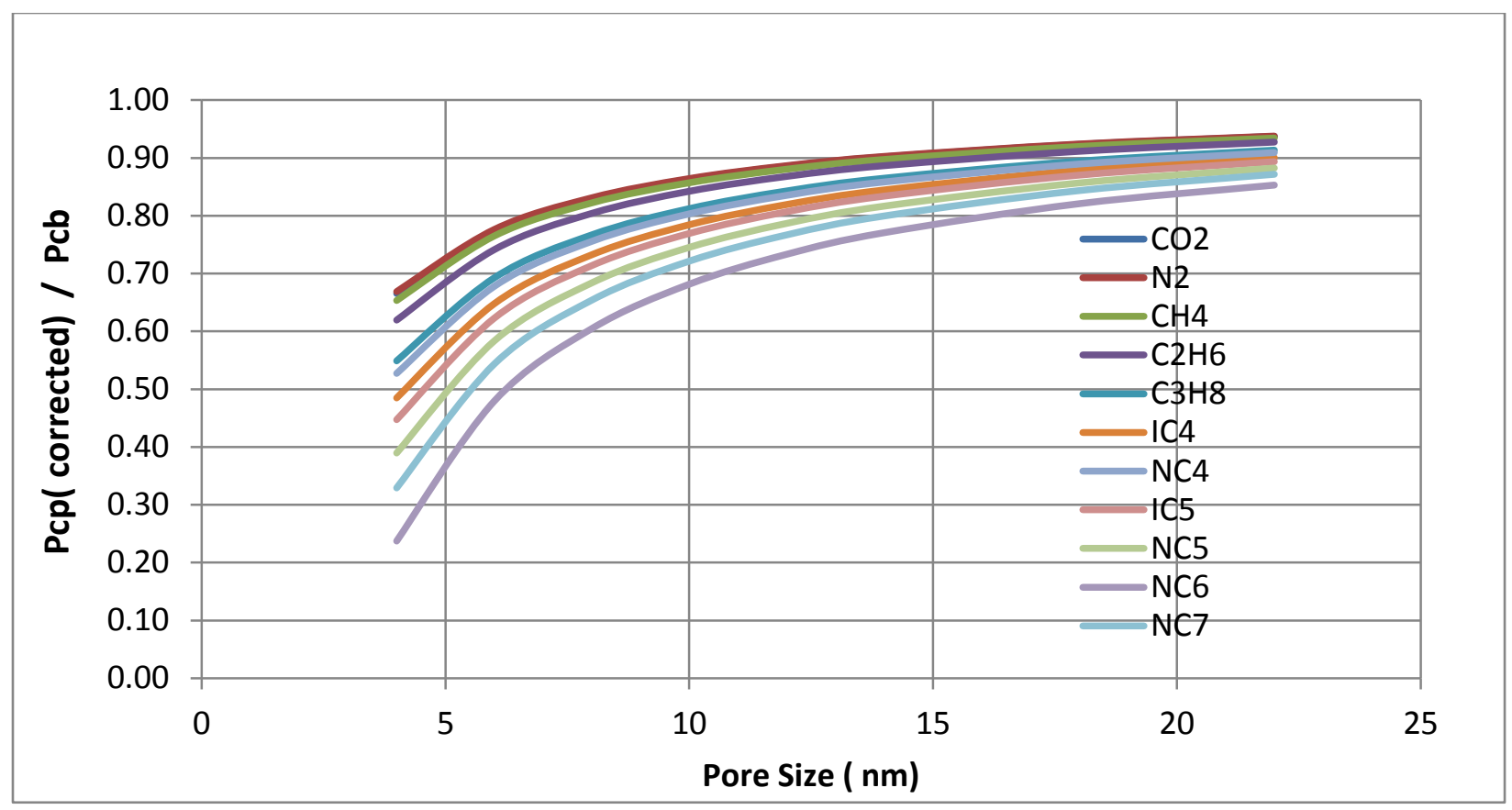

Figure 25 : The percentage of critical Pressure deviation from the bulk value for pure components using the corrected semi-analytical solution at a range of pore sizes

\section{3: Phase Envelope Calculation of the Gas Mixture inside the Organic Nano-Porous Media:}

Different techniques have been presented to derive the mixing rules from the principles of statistical mechanics or merely classical thermodynamic arguments. However, quadratic mixing rules, originally proposed by Van der Waals, are used extensively in mixture calculations involving equations of state [Hall et. al., 1993 ]. As it is suggested in the CMG simulator Ver.2012, this method was employed with our modification of Van der Waals equation of state to obtain the two phase envelopes at different average nano-pore sizes. Figure 26 below shows how the phase envelope changes as the pores are getting smaller and smaller due to the effect of wall pore confinement. The effective pore size in our case was $10 \mathrm{~nm}$. And the max pore size at which the effect of the confinement was significant was $100 \mathrm{~nm}$. More than that, the fluid model acts just like an unconfined case. 


\begin{tabular}{|c|c|c|c|c|c|c|c|c|c|c|c|c|}
\hline \multirow{3}{*}{ Component } & \multirow[b]{2}{*}{ T Bulk } & \multirow[b]{2}{*}{ P Bulk } & \multicolumn{2}{|c|}{$4(\mathrm{~nm})$} & \multicolumn{2}{|c|}{$6(\mathrm{~nm})$} & \multicolumn{2}{|c|}{$8(\mathrm{~nm})$} & \multicolumn{2}{|c|}{$10(\mathrm{~nm})$} & \multicolumn{2}{|c|}{$100(\mathrm{~nm})$} \\
\hline & & & Tc & Pc & Tc & Pc & Tc & $\mathrm{Pc}$ & Tc & Pc & Tc & $\mathrm{Pc}$ \\
\hline & $\mathrm{K}$ & atm & $\mathrm{K}$ & atm & $\mathrm{K}$ & atm & $\mathrm{K}$ & atm & $\mathbf{K}$ & atm & $\mathrm{K}$ & atm \\
\hline $\mathrm{CO} 2$ & 304.2 & 72.8 & 289.5 & 48.4 & 294.3 & 56.4 & 296.7 & 60.4 & 298.2 & 62.8 & 303.6 & 71.8 \\
\hline 2 & 126.2 & 33.5 & 119.2 & 22.4 & 121.5 & 26.0 & 122.6 & 27.8 & 123.3 & 29.0 & 125.9 & 33.0 \\
\hline $\mathrm{CH} 4$ & 190.6 & 45.4 & 174.7 & 29.7 & 179.8 & 34.7 & 182.4 & 37.3 & 184.0 & 38.9 & 189.9 & 44.7 \\
\hline $\mathrm{C} 2 \mathrm{H} 6$ & 305.4 & 48.2 & 274.8 & 29.9 & 284.6 & 35.7 & 289.6 & 38.8 & 292.7 & 40.6 & 304.1 & 47.4 \\
\hline $\mathrm{C} 3 \mathrm{H} 8$ & 369.8 & 41.9 & 331.5 & 23.0 & 343.7 & 29.0 & 350.0 & 32.1 & 353.9 & 34.0 & 368.2 & 41.1 \\
\hline IC4 & 408.1 & 36.0 & 360.7 & 17.5 & 375.7 & 23.3 & 383.5 & 26.4 & 388.3 & 28.2 & 406.1 & 35.2 \\
\hline $\mathrm{NC4}$ & 425.2 & 37.5 & 383.2 & 19.8 & 396.6 & 25.4 & 403.5 & 28.3 & 407.7 & 30.1 & 423.4 & 36.7 \\
\hline IC5 & 460.4 & 33.4 & 416.9 & 14.9 & 430.7 & 20.8 & 437.8 & 23.8 & 442.2 & 25.7 & 458.5 & 32.6 \\
\hline NC5 & 469.6 & 33.3 & 429.4 & 13.0 & 442.1 & 19.4 & 448.8 & 22.8 & 452.8 & 24.8 & 467.9 & 32.4 \\
\hline NC6 & 507.4 & 29.3 & 471.5 & 7.0 & 482.9 & 14.0 & 488.8 & 17.7 & 492.4 & 20.0 & 505.9 & 28.3 \\
\hline NC7 & 540.2 & 27.0 & 506.2 & 8.9 & 517.1 & 14.7 & 522.7 & 17.7 & 526.1 & 19.5 & 538.8 & 26.2 \\
\hline
\end{tabular}

Table 3: Critical parameters calculation results with new consideration for different pore sizes

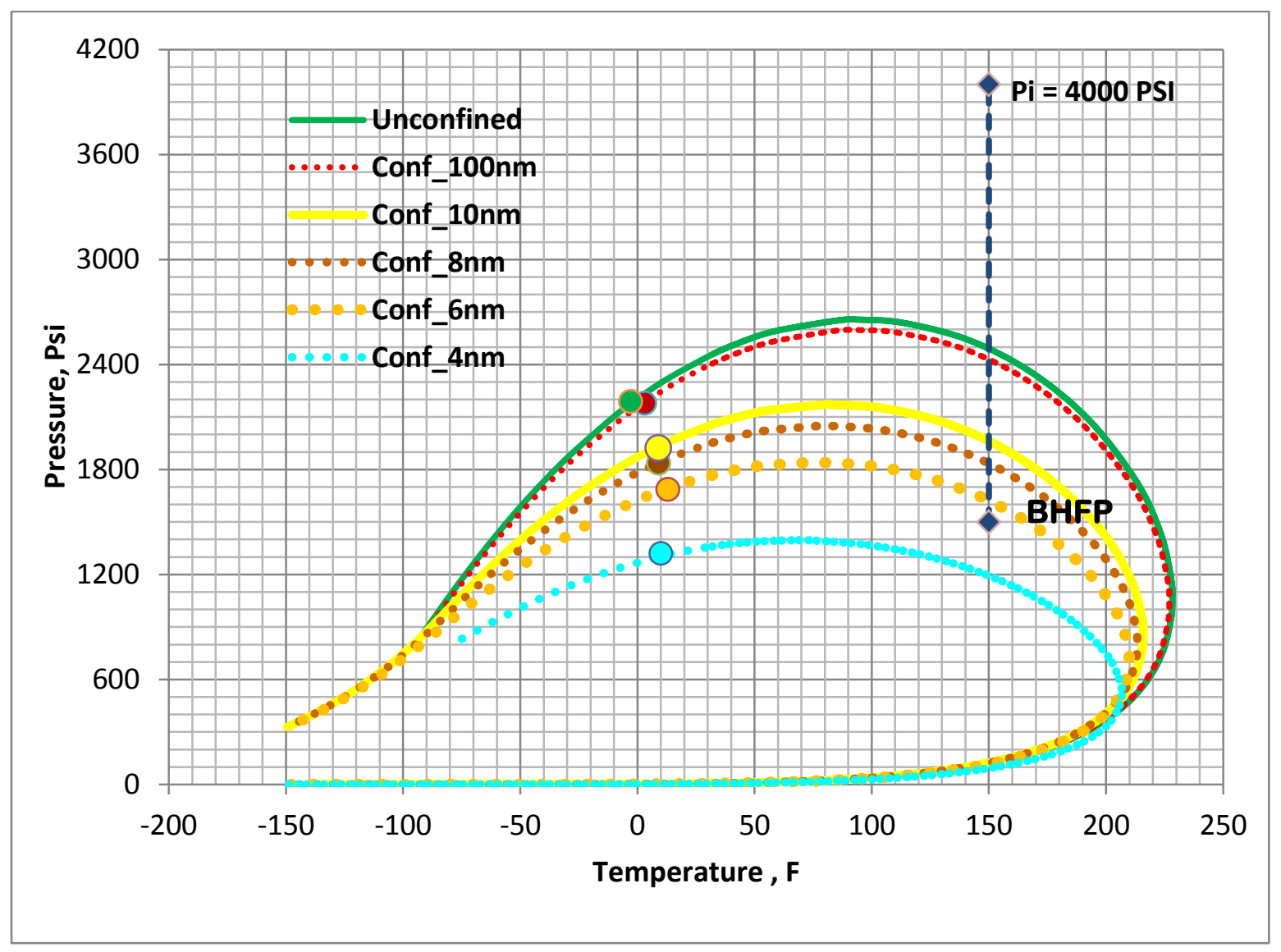

Figure 26 : Phase envelope changes at range pore sizes using the base case fluid model. 


\section{4: Reservoir and Fluid Characteristics Effects on the Condensation Bank Development.}

In addition to reservoir fluid components, their mole fractions, initial and bottom hole pressures, number of horizontal wells and hydraulic fracturing spacing the reservoir characteristics play an important role in condensation distribution and condensate buildup mechanisms inside the reservoir that has to be investigated. Five parameters were chosen in this study to represent the most important factor affecting the condensate shale reservoir. Those parameters are matrix anisotropic factor, the absolute permeability, natural fracture density, porosity, and rock compressibility.

\section{5: Operational and Completion Condition Effects on the Condensation Bank Development.}

Because of the disability of such reservoirs to produce naturally at economic rates due to the ultra-low permeability. It is necessary to use the horizontal well drilling and the hydraulic fracturing stimulation to increase contact surface between wells and reservoir matrix. Here our interest focused on the effect of the number of horizontal wells and the hydraulic fracture spacing on the condensate bank development and saturation profiles. In addition to the fluid and the reservoir parameters, the operational condition also significantly impacts the long term production performance of the condensate reservoirs. Considering this effect, the pressure difference between reservoir initial pressure and operating constant bottom hole pressure is also considered as operational parameter that can influence the dynamics of condensate bank development.

\section{6: Simulation:}

To achieve the aim of this study that is the understanding of gas condensation dynamics in organic rich shale the, CMG simulator has been employed. For this purpose two study cases were proposed, a synthetic case and a realistic case, with an actual fluid model. The cylindrical model (synthetic case) utilized with one vertical well and a homogenous lithology to imitate a typical well in the gas shale formation. This case was built just for scanning test to eliminate some of the candidate parameters. The actual 
reservoir model was utilized by variety of vertical, horizontal and hydraulic fractured wells in a standard development pattern. All different lithology maps that used to build the model are documented in the appendix A. As well as the results of different simulations are discussed.

\subsection{1: Fluid Model Description:}

A real fluid model was introduced for this simulation with variety of components to resemble a condensate gas mixture occupied the Marcellus shale reservoir. The composition simulator was utilized with Win Prop application to host these data. Base case reservoir fluid mixture contains nine components of reservoir fluid from methane to hexane and heavy components lumped in to heptane plus. Non-hydrocarbon components including carbon dioxide and nitrogen are also presented in this fluid mixture. A detailed composition of the mixture and their mole fractions are listed in Table 4. Figure 27 shows the phase envelope of the reservoir fluid.

\begin{tabular}{|c|c|}
\hline \multicolumn{2}{|c|}{ Condensate Fluid Model } \\
\hline \hline \multirow{2}{*}{ Components } & Composition \% \\
\hline \hline CO2 & 1.24 \\
\hline N2 & 0.34 \\
\hline C1 & 79.67 \\
\hline C2 & 5.47 \\
\hline C3 & 3.96 \\
\hline IC4 & 1.1 \\
\hline NC4 & 1.52 \\
\hline IC5 & 0.79 \\
\hline NC5 & 0.63 \\
\hline C6 & 1.13 \\
\hline C7 & 4.15 \\
\hline Total & 100 \\
\hline
\end{tabular}

Table 4: Mixture components details [ Imo-Jack, 2010]. 


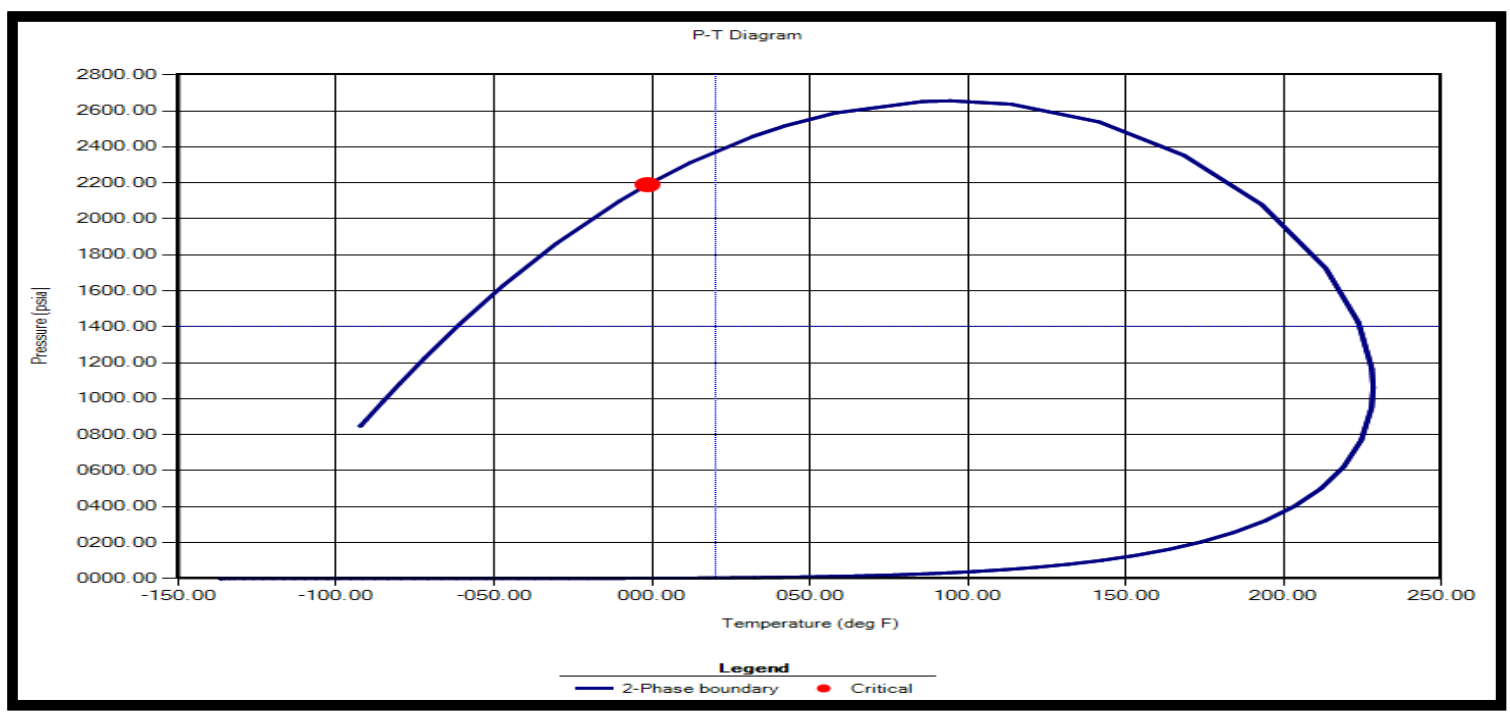

Figure 27: The Two Phase envelop of the mixture [ WinProp 2012].

\subsection{2: Case Study 1:}

This is a synthetic case to investigate the gas condensate dynamics in a simple model. A cylindrical model was used in this case to resemble a single vertical well unconventional gas reservoir. Model includes 10*100 grids with 7 layers. Homogeneous reservoir properties assigned in this case. The reservoir specification is listed in table 5 below; and a 3-D top view of the whole grid was shown in Figure 28.

\begin{tabular}{|l|c|}
\hline \multicolumn{2}{|c|}{ Reservoir Description forsimulation } \\
\hline Drainage Area, ftz & 7850 \\
\hline Thickness (h), ft & 600 \\
\hline Absolute permeability (k), 0 & 300 \\
\hline porosity (f), fraction & 0.015 \\
\hline Initialpressure (pi), psi & 4000 \\
\hline Dempointpressure (pd), psi & 2538 \\
\hline Temperature, of & 150 \\
\hline Compressibility (ct), psi-1 & 26 \\
\hline \hline
\end{tabular}

Table 5: The reservoir specification (the synthetics case) 


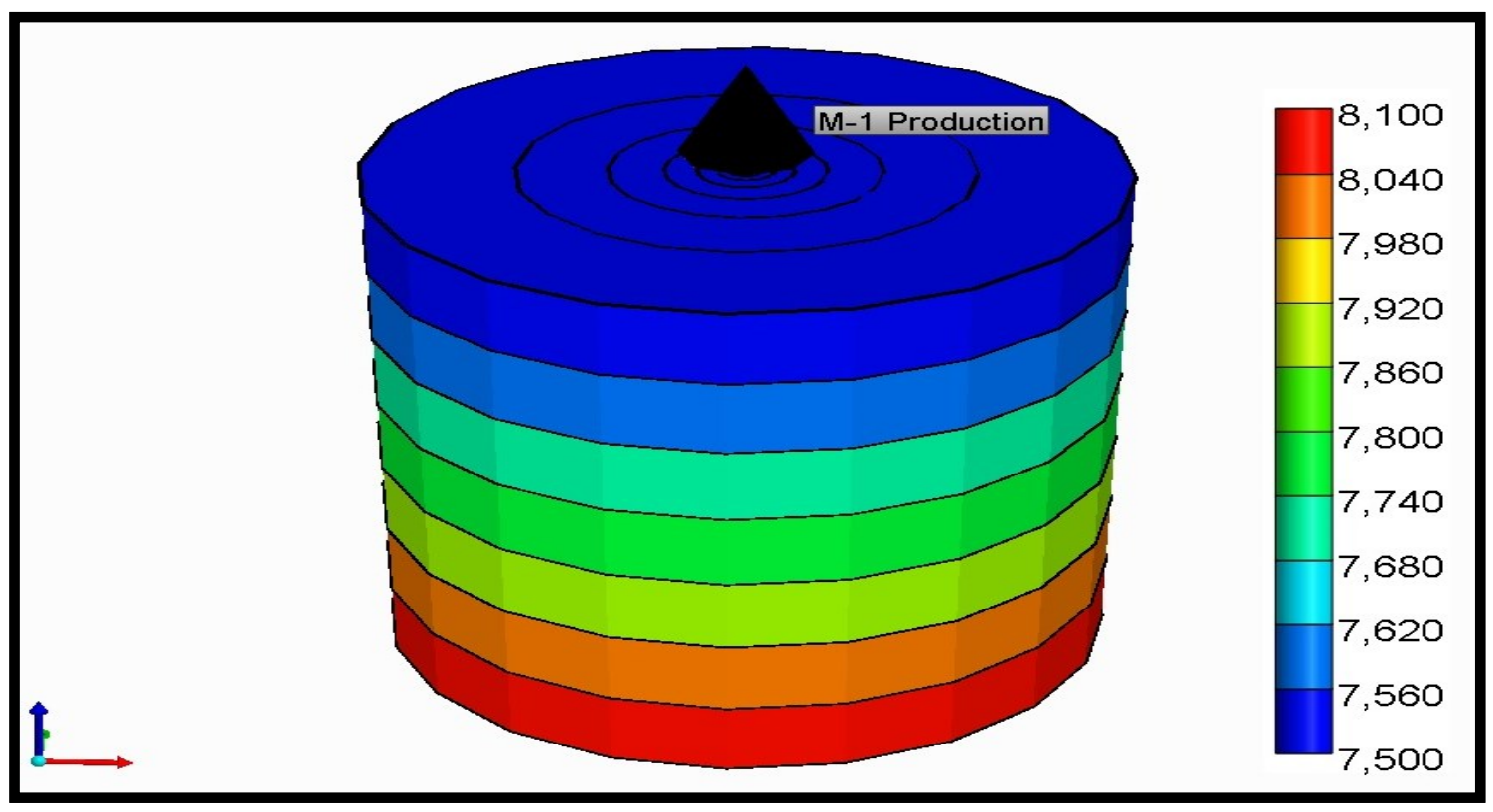

Figure 28: The depth top grid view for the reservoir model.

\subsection{3: Case Study 2:}

In this case an actual model was built to resemble real properties of the Marcellus shale gas reservoir. The grid was $40 * 40$ with about $100 \mathrm{ft}$ layer thickness at approximately $7600 \mathrm{ft}$ depth. Heterogeneous reservoir rock properties are defined using actual structure, depth, porosity and permeability distribution maps were presented in the appendix $A$, figures (A1.1-4). Summary of the characteristics of this model is listed in table 6 . Also, figure (29) shows $3 \mathrm{D}$ and $2 \mathrm{D}$ view of the reservoir complete model. 


\begin{tabular}{|l|c|}
\hline \multicolumn{2}{|c|}{ Reservoir Description for Simulation } \\
\hline \hline Drainage Area (ft2) & $\approx 9700$ \\
\hline Reservoir length(ft) & 22273 \\
\hline Reservoir Width(ft) & 18967 \\
\hline Thickness (ft) & $80-124$ \\
\hline Absolute Permeability (k) (nD) & $131-710$ \\
\hline Porosity (f) & $1.16-8.1$ \\
\hline Initial Pressure (pi) & 4000 \\
\hline Dew point Pressure (pd) & 2538 \\
\hline Temperature & 150 \\
\hline Compressibility (ct), psi-1 & $2 \mathrm{E}-6$ \\
\hline
\end{tabular}

Table 6: The reservoir details (the actual case).
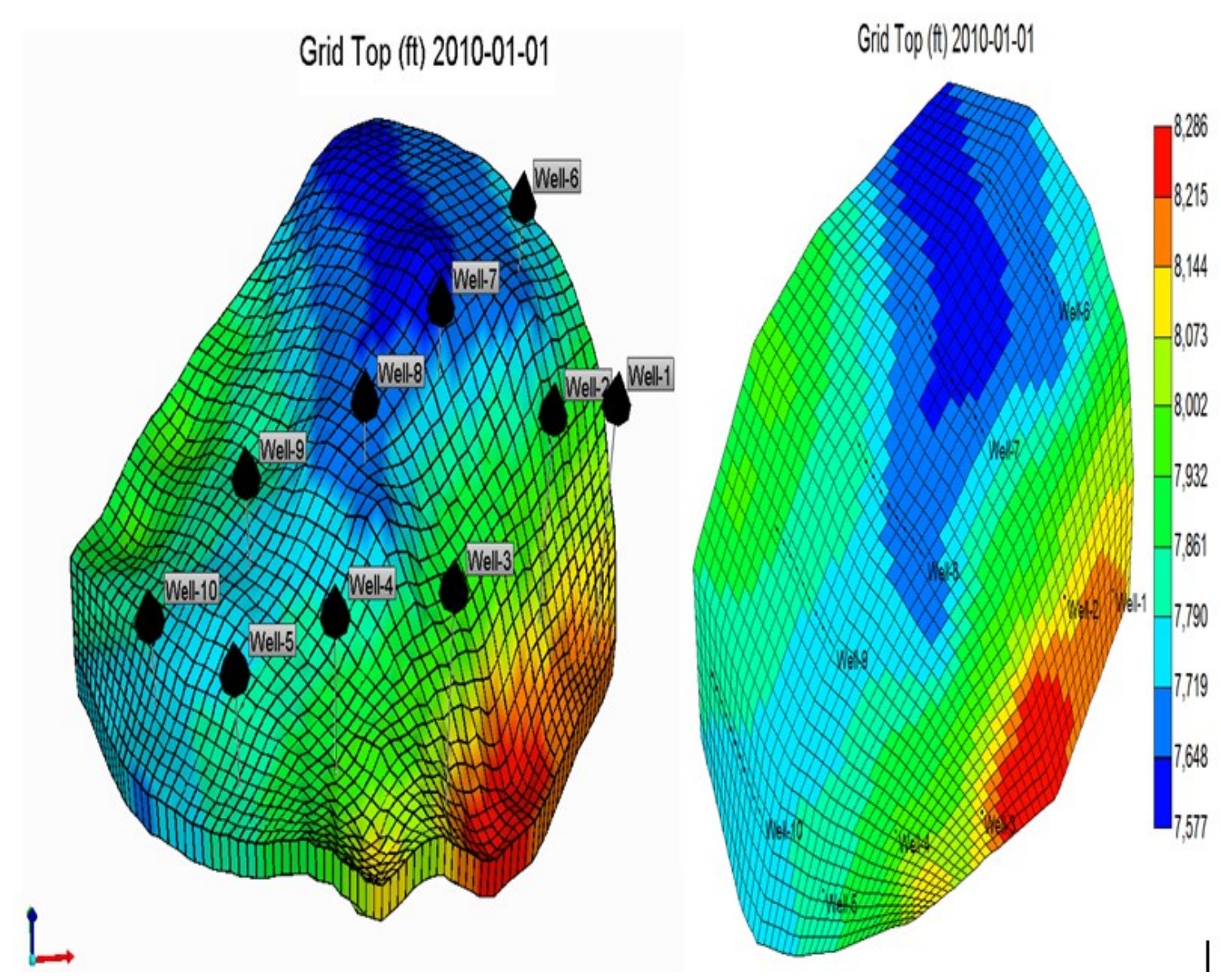

Figure 29: The grid top view (3D \& 2D) of the base case model (the actual model) 


\section{7: Sensitivity Analysis:}

Due to the presence of different variables contributing in the gas condensation dynamics, it is very difficult to identify the specific role played by each one of these parameters on condensation build up and its dynamics around the wells. Modern techniques like design of experiments help to quantify the contribution of the candidate parameters as well as their interactions.

\subsection{1: Design of Experiments (DOE):}

The main derive to use experimental design which is a technique for uncertainty analysis of the process is due lack of sufficient knowledge of the process, lack of sufficient precision in obtaining the parameters of interest and finally lack of control on interaction between different parameters that results in complicated process. Due to complexity of the problem and impact of different parameters contributing to gas condensation dynamics, it's not possible to use conventional technique where all the variables are assumed to be fixed while sensitivity analysis is performed on one variable, i.e. One Variable At the Time (OVAT). In OVAT technique the parameters are assumed to be independent however here the parameters of interest are dependent in a direct or reverse manner. In this technique the response line will be generated and used for interpolation of the effects. Different experimental design techniques introduced based on the number of variables under study and their level of change. It is common to assume two or three levels of change in variables. In case of two levels change in a variable only high, commonly presented with +1 , and low level, represented by -1 , is used while considering three levels of changes for variables the middle level or zero level is also considered. In some cases comprehensive design of experiments will be conducted that is the Full Factorial Design (FFD). FFD encompasses all possible combinations of the variables. In this case the total number of cases "Runs" is given by $L^{N}$ where $L$ denotes the number of levels for each variable and $N$ is the number variables. These designs are expensive and take long time to run; on the other side they can acquire high accuracy results. However, later attempts have been made to minimize the number of runs while maintaining the resolution as high as possible at the same time. 
They take into account all possible combination of 2 or 3 level values for unlimited number of factors. The representation of these tests is a surface of interaction which is known as a response surface model (RSM analysis). In this study we use five step systematic approach to expose information about the most important features of the problem studied, where we first determine the parameters of interest and their levels of variation, i.e., Tables 8 and 9 for the cylindrical and the actual reservoir model. Then we perform a linear screening analysis to determine the most important parameters using special design with two levels of variation called Plackett-Burman design. The nonlinear behavior of the variables and simulations of response surface will be performed next. Finally the optimum response surface will be obtained using commercial software Design-Expert. Table 7 shows the change in base case reservoir fluid which considered in this study for lean composition, i.e. low level or (-1), and rich composition, i.e., high level or $(+1)$.

\begin{tabular}{|l|c|c|c|}
\hline \multicolumn{1}{|c|}{ Comp. } & \multicolumn{3}{c|}{ Mole Fraction } \\
\cline { 2 - 4 } & Lean (-1) & Base Case & Rich (-1) \\
\hline \hline $\mathrm{CO}_{2}$ & 0.0131 & 0.0124 & 0.012 \\
\hline $\mathrm{N}_{2}$ & 0.0088 & 0.0034 & 0.0034 \\
\hline $\mathrm{C}_{1}$ & 0.8168 & 0.7967 & 0.775 \\
\hline $\mathrm{C}_{2}$ & 0.0579 & 0.0547 & 0.052 \\
\hline $\mathrm{C}_{3}$ & 0.0415 & 0.0396 & 0.0314 \\
\hline $\mathrm{IC}_{4}$ & 0.0117 & 0.011 & 0.011 \\
\hline $\mathrm{NC}_{4}$ & 0.0162 & 0.0152 & 0.0152 \\
\hline $\mathrm{IC}_{5}$ & 0.004 & 0.0079 & 0.0189 \\
\hline $\mathrm{NC}_{5}$ & 0.0032 & 0.0063 & 0.0193 \\
\hline $\mathrm{C}_{6}$ & 0.0057 & 0.0113 & 0.0163 \\
\hline $\mathrm{C}_{7}$ & 0.0211 & 0.0415 & 0.0455 \\
\hline Total & 1 & 1 & 1 \\
\hline
\end{tabular}

Table 7: Fluid model variation for sensitivity analysis. 


\begin{tabular}{|l|l|c|c|c|}
\hline Factor & Parameter & Hi (+1) & Base Case & Low (- 1) \\
\hline No. & & 3000 & 2750 & 2500 \\
\hline Factor 1 & $\Delta \mathbf{P}$ & rich & Base Case & Lean \\
\hline Factor 2 & Composition & 700 & 0.0003 & 70 \\
\hline Factor 3 & Absolute Permeability & 0.12 & 0.015 & 0.015 \\
\hline Factor 4 & Porosity & $2.00 E-04$ & $2.00 E-05$ & $2.00 E-06$ \\
\hline Factor 5 & Rock Compressibility PsI ${ }^{-1}$ & 2.005 \\
\hline
\end{tabular}

Table 8: Candidate parameters variation ( $\mathrm{Hi}$ and low values) for the cylindrical reservoir model

\begin{tabular}{|c|c|c|c|c|}
\hline $\begin{array}{c}\text { Factor } \\
\text { No. }\end{array}$ & Parameter & Hi $(+1)$ & $\begin{array}{l}\text { Base } \\
\text { case }\end{array}$ & Low $(-1)$ \\
\hline Factor 1 & $\Delta \mathbf{P}$ & 3000 & 2750 & 2500 \\
\hline Factor 2 & Composition & Rich & Base case & Lean \\
\hline Factor 3 & No. Of Horizontal Wells & 10 & 5 & 5 \\
\hline Factor 4 & Hydraulic Fracture Spacing (ft) & 1200 & none & 500 \\
\hline Factor 5 & Natural Fracture Density (Frac/ft) & 10 & 4 & 2 \\
\hline Factor 6 & Matrix Anisotropic Factor & 1.4 & 1 & 0.6 \\
\hline Factor 7 & Rock Compressibility PSI ${ }^{-1}$ & 2.00E-04 & 2.00E-05 & 2.00E-06 \\
\hline
\end{tabular}

Table 9: Candidate parameters variation (Hi and low values) for the actual reservoir model.

The response parameter in our experiments is damage zone around different vertical and horizontal wells which was determined from each case by detecting the time when condensation starts and the distance from the well at which the effective condensate saturation expands. For the purpose critical oil saturation is obtained using proper relative permeability curve and minimum condensate saturation for damage zone is considered to be $10 \%$. Figure 30 (a) and (b) clearly shows the steps to find the damaged zone around the wellbore comparing the saturations with critical oil saturation mentioned earlier. In graph (a) the time the condensate started in the reservoir detected when the GOR curve starts to increase significantly, which is a direct indicator of losing the heavy components inside the reservoir. In graph (b) the condensation saturation vs. distance was plotted at condensation starting time to locate the damaged zone. Eventually, the determined damage zone was used as a response input for each run in the experimental design model. 

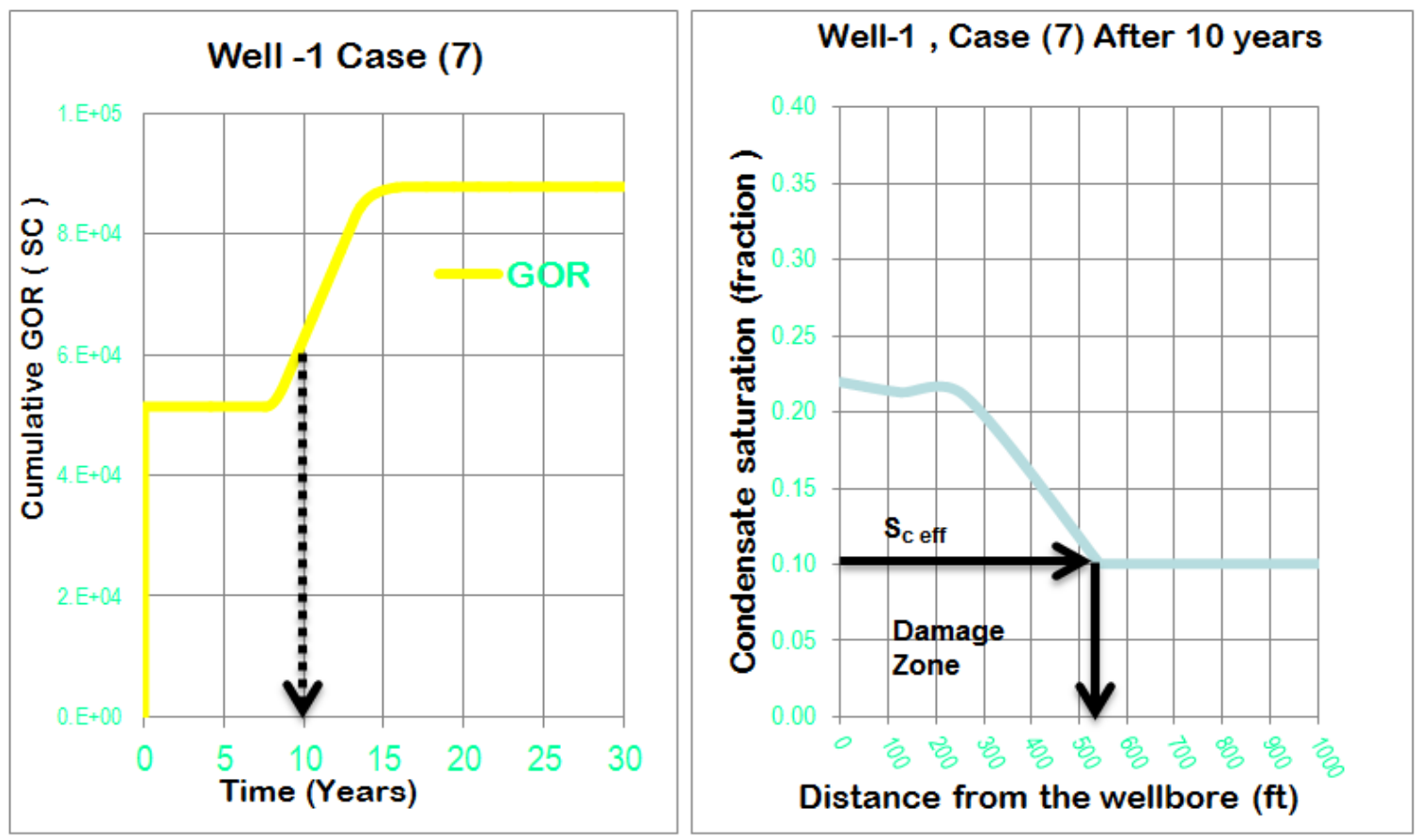

Figure 30: Determining the damage zone due to the condensate bank development using the GOR Plackett-Burman Design Model (PBD):

Non-regular designs are a variety of designs that are widely used because they allow for a little more flexibility in run size. Two-level FFDs are severely limited in choices of run size because they must be powers of 2 . Non-regular designs help alleviate this problem by allowing for better management of run size economy. Some of the most widely used non-regular designs are Plackett-Burman designs. This model provides the maximum information about a system in minimum number of runs. PBD requires $(n+1)$ experiments (runs), where ( $n$ ) is the number of variables and it is only available in multiples of 4 e.g. 11 factors can be analyzed by a 12-run PB design but 9 factors will also need a 12-run PB design. Primarily 4 design resolutions have been defined. Resolution II where main effects will be confound with each other. Resolution III does not confound main effects with each other but confounds main effects with two-factor interactions. Resolution IV where two factor interactions only confound with other two factor interactions and finally Resolution $\mathrm{V}$ where only two factor interactions are confound with three factor interactions. This model generates a design of resolution (III). 
This means all the main effects can be analyzed. This experimental plan can help in narrowing down the number of the significant factors so it becomes easier to run a 3level comprehensive test among them. However, although this design categorized under the screening designs, it is still a successful method to deal with the uncertainty when there are not a large number of potential factors interacting together in a process.

Table 10 shows the details of different runs following PBD for 7 reservoir parameters that have been selected using pre-screening technique.

\begin{tabular}{|c||c||c||c||c||c||c||c|}
\hline Run & Factor 1 & Factor 2 & Factor 3 & Factor 4 & Factor 5 & Factor 6 & Factor 7 \\
\hline \hline No. & $(\Delta \mathrm{P})$ & $(\mathrm{C} 5+)$ & \# H. Well & $\begin{array}{c}\text { H.Frac. } \\
\text { Spacing }\end{array}$ & $\begin{array}{c}\text { N. Frac } \\
\text { Density }\end{array}$ & $(\mathrm{Kv} / \mathrm{Kh})$ & $(\mathrm{Cp})$ \\
\hline \hline 1 & -1 & -1 & -1 & 1 & 1 & 1 & -1 \\
\hline 2 & 1 & -1 & -1 & -1 & -1 & 1 & 1 \\
\hline 3 & -1 & 1 & -1 & -1 & 1 & -1 & 1 \\
\hline 4 & 1 & 1 & -1 & 1 & -1 & -1 & -1 \\
\hline 5 & -1 & -1 & 1 & 1 & -1 & -1 & 1 \\
\hline 6 & 1 & -1 & 1 & -1 & 1 & -1 & -1 \\
\hline 7 & -1 & 1 & 1 & -1 & -1 & 1 & -1 \\
\hline 8 & 1 & 1 & 1 & 1 & 1 & 1 & 1 \\
\hline
\end{tabular}

Table 10: Plackett-Berman matrix for seven variables, $(-1)=$ Low Value; $(+1)=$ Hi Value

\subsection{2: Experimental Design Analysis:}

Using commercial software called Design-Expert ver.8.1; it is clear to visualize the results obtained from the experimental design model used in this study. We analyzed the results using Pareto chart, normal plot of the standardized effects and 3-D surface response. The Pareto chart displays the relative size of effects and present the importance of the parameters in descending order as it can be seen in Figure 31. It uses dimensionless statistics to scale the effects in terms of standard deviations. The t-value is one of those statistics that related to the size of the difference between the means of the two samples being compared. The larger $t$ is, the larger the difference. In this design 
Pareto chart analyzes the uncertainty into three classification: parameters are almost certainly significant, positive or negative recognized by the color, when they appear above the Bonferroni Limit, which is simply the alpha level divided by the number of estimates being made for the purpose of testing simultaneous significant effects; parameters are less significant contribution in the response when they are between the tValue, which is calculated by the mean difference divided by the Standard Error, and Bonferroni limit; and finally, parameters are not important when they show up under the T-value limit. The greater t-level means the lower confidence in the analysis.

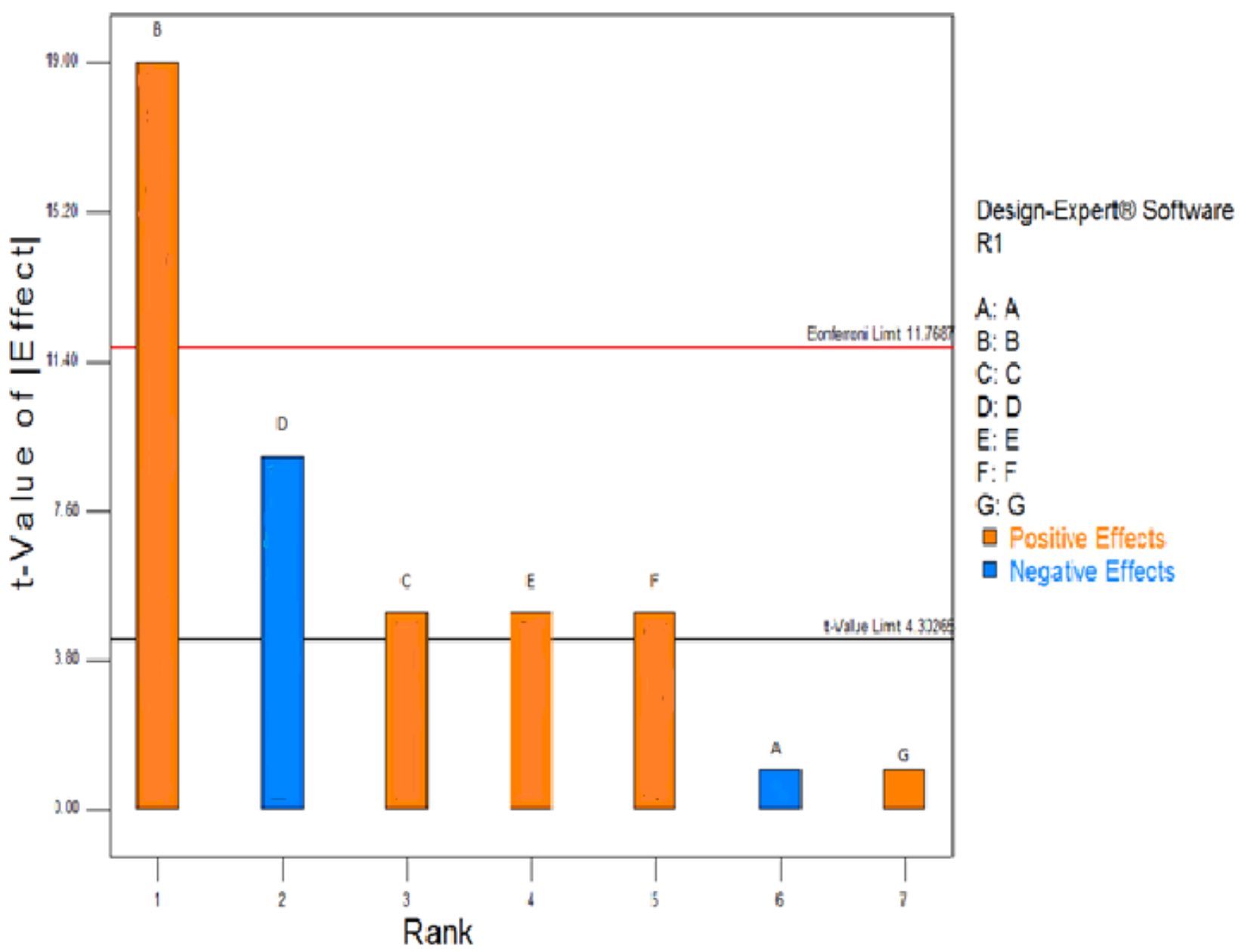

Figure 31: Pareto chart shows the importance of parameters evaluated on t-value. It displays a general example for seven synthetic factors (A-G) were sorted based on the chart rule. 
The normality plot is shown in Figure 32 displays the magnitude and the direction of the standardized effects. These effects evaluate the average response from the parameter at high and low value. The normality test in this design is called Shapiro-Wilk that is a statistical test run on the desired terms to determine if they follow a normal distribution. Another important term on this plot is the $p$-value that helps the analyzer to decide whether or not to accept the null hypothesis. You make this decision by deciding how low the $p$-value should be before you will reject the null hypothesis. This cut-off point is called the significance level and is usually set at 0.1 .

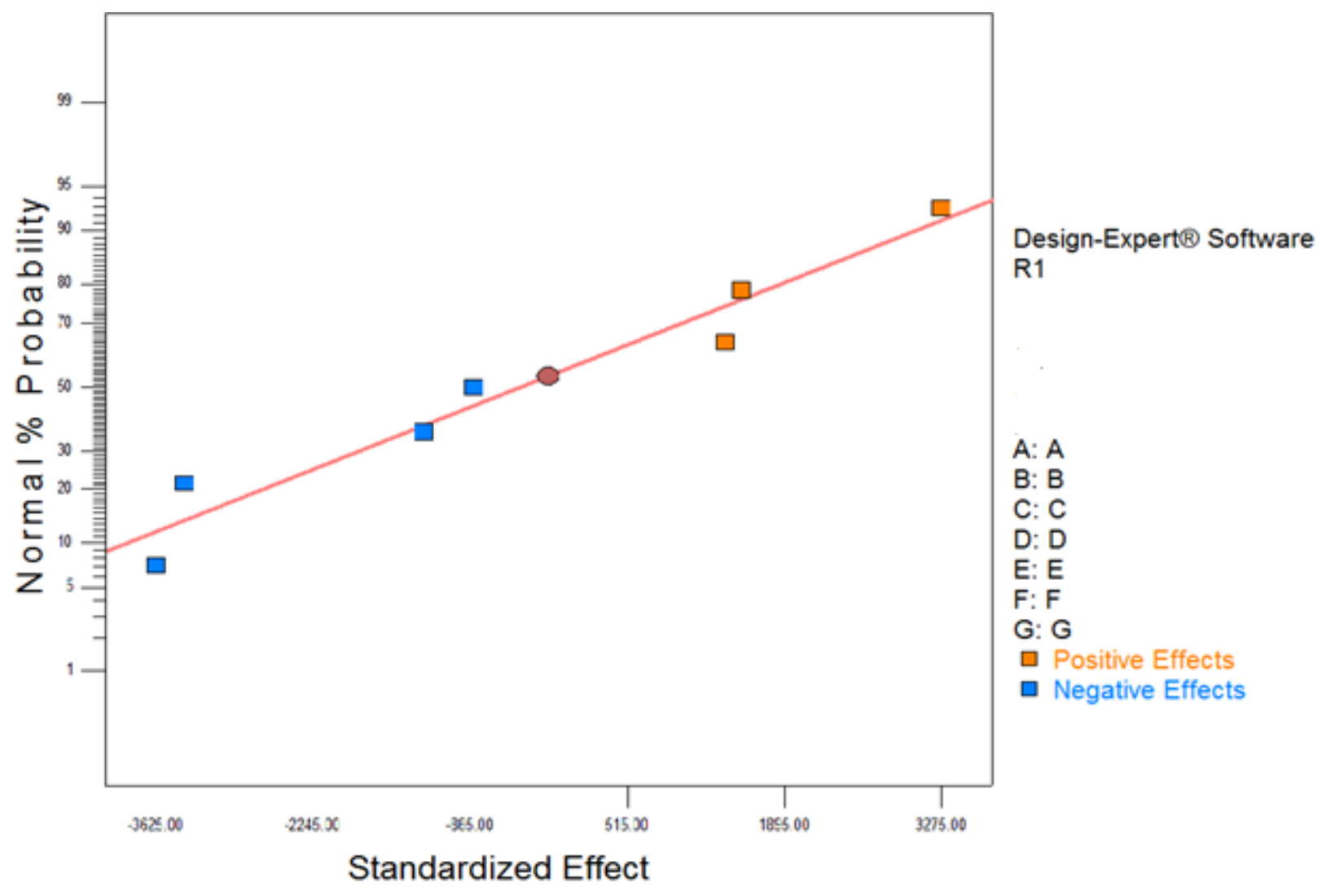

Figure 32: The normality test of the parameters shows a normal distribution.

The Surface response, which demonstrated in Figure 33 and Figure 34, gives the visualization for the individual and the interactions effects on the respond value in 3-D and contour lines using the actual values for the factors codes. (Design-Expert manual). 


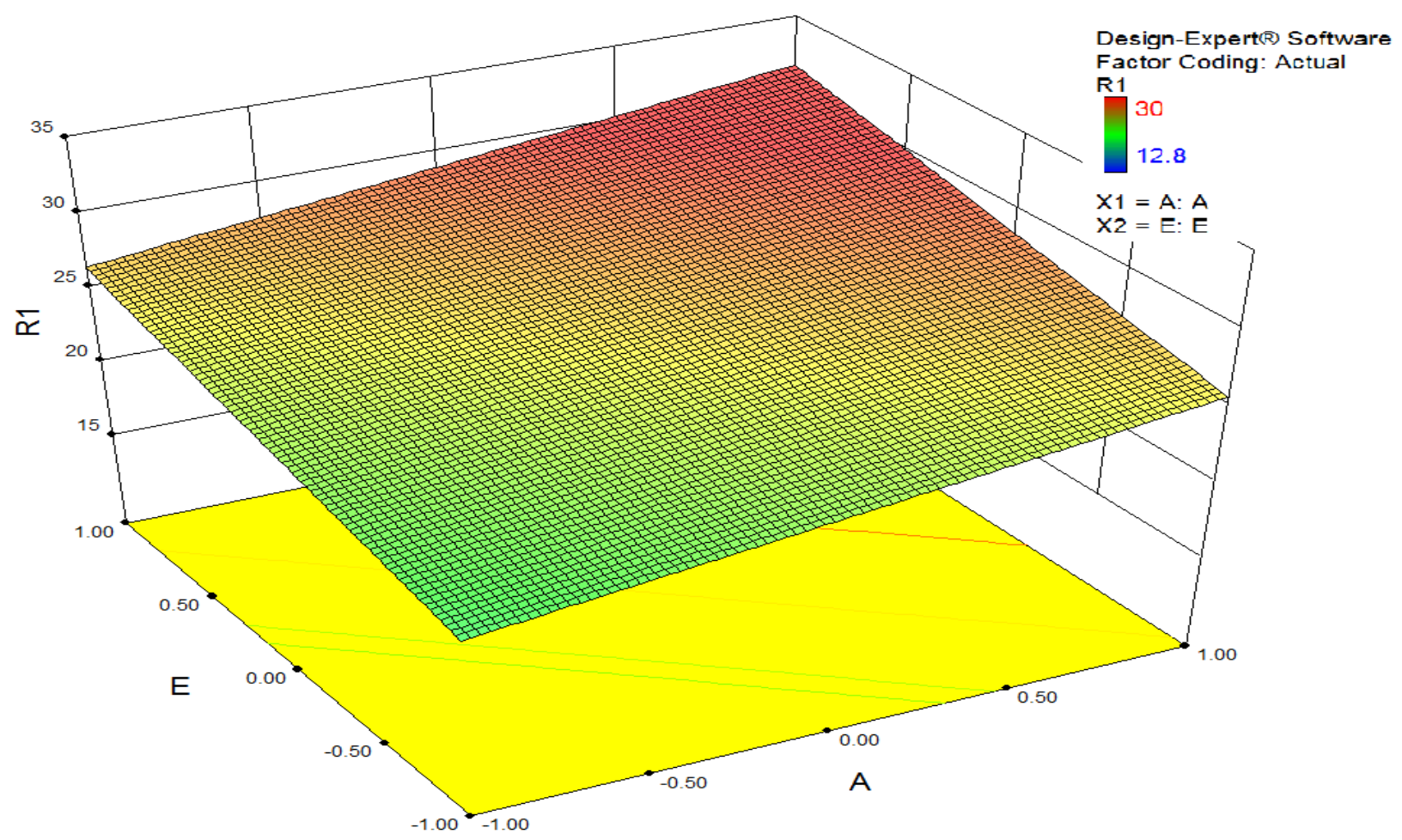

Figure 33: 3-D surface generated using the color variation to visualize the magnitude and trend and the parameters impact on the response value

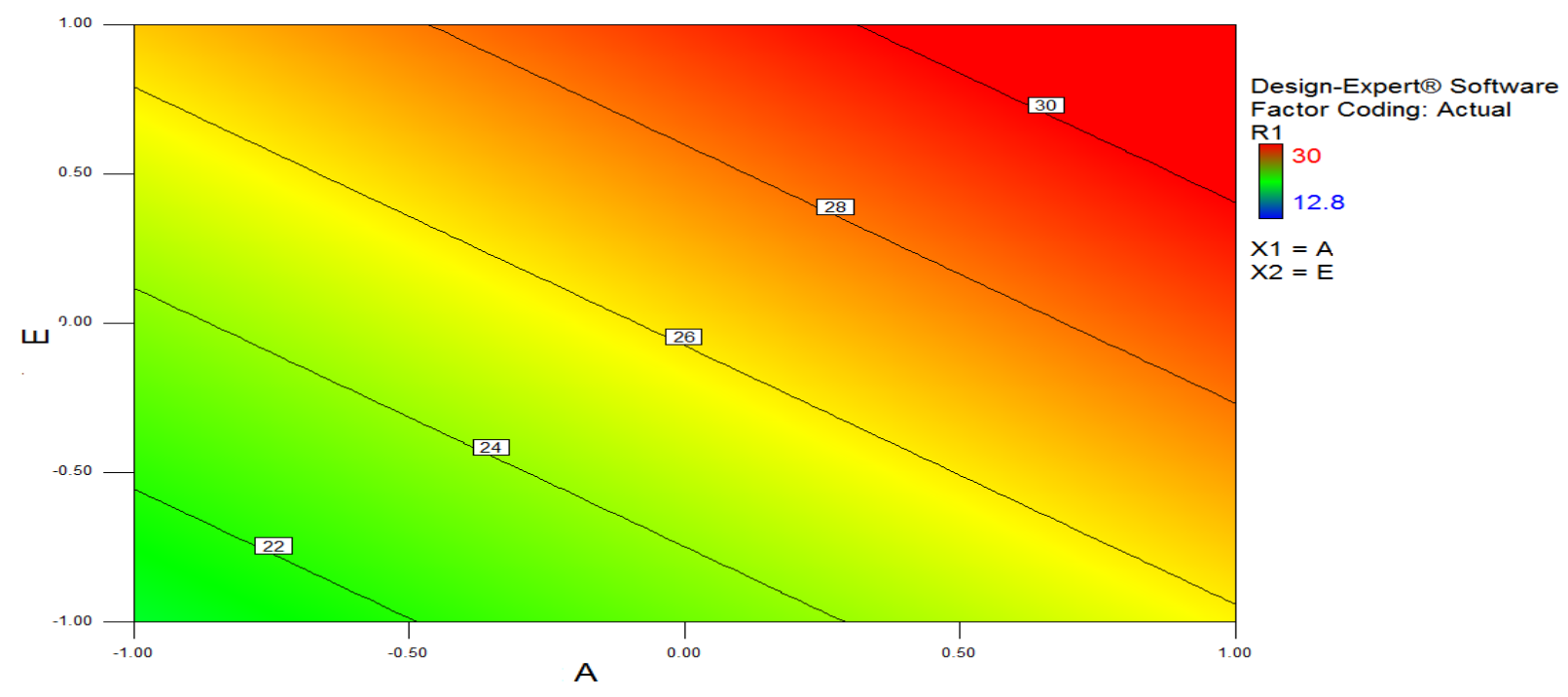

Figure 34: 3-D surface generated using the contour lines to show the magnitude and trend and the parameters impact on the response value 


\section{CHAPTER: 4: RESULTS AND CONCLUSIONS}

\section{1: Results and discussion}

Figure 35 brings up a comparison between the phase envelope for unconfined case and a phase envelope generated for confined case using the base case fluid model presented in Table 2. It indicates that the dew point of 2500 psi at which the condensate starts to happen at the unconfined case is reduced to a value less than the 2000 psi. That gives more time for the reservoir to maintain producing gas above the dew point. Therefore leaves more room to drop the bottom hole pressure and apply more pressure gradient increasing the well productivity. However, in the unconfined case where we ignore the pore wall confinement effects the dew point is higher and therefore the aggression of the liquid dropout is more.
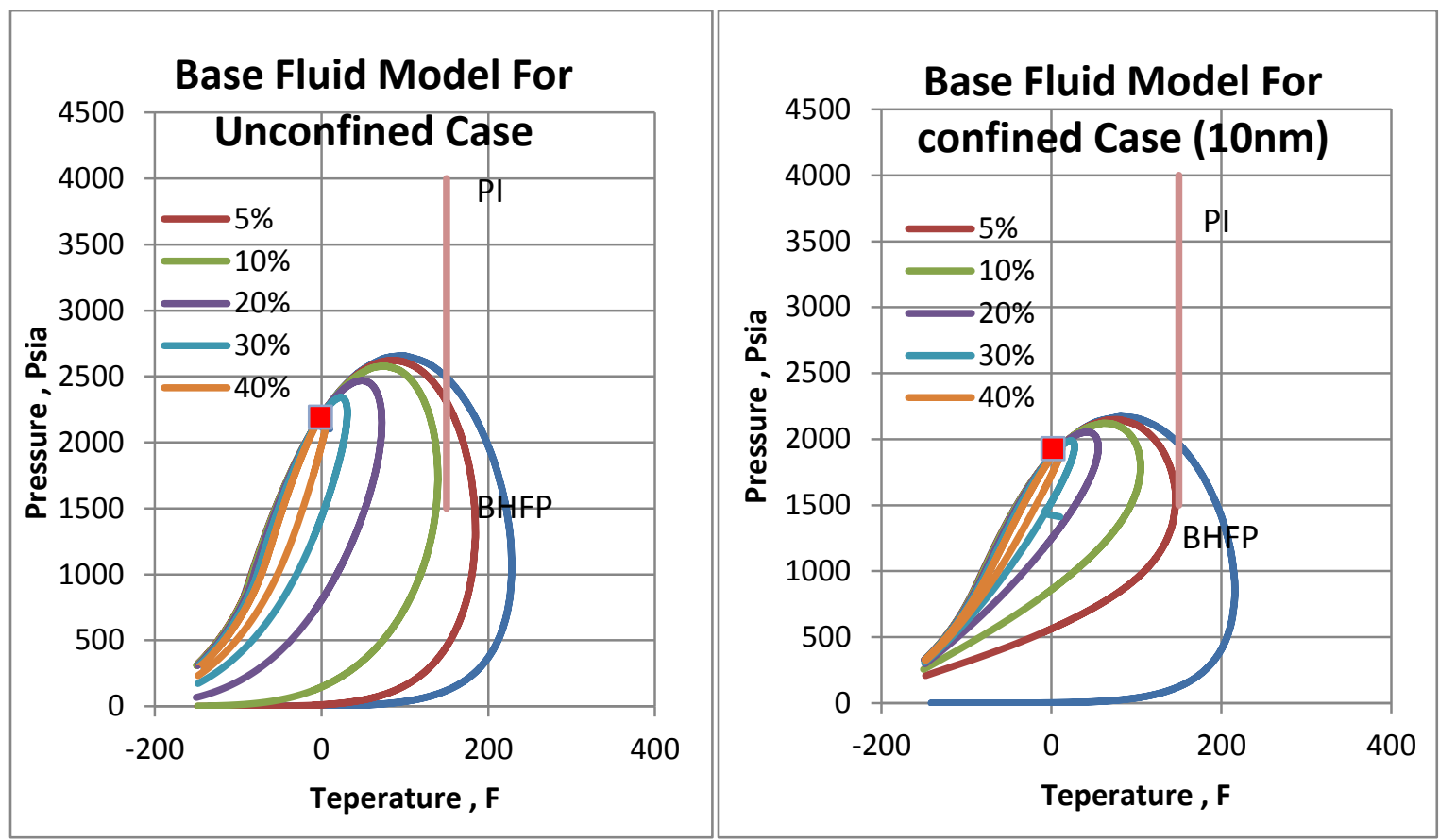

Figure 35: Liquid dropout volume percentage for the base case fluid model considering the well pore approximation. 
By analyzing the relative permeability curves in figure 36 , provided for the geologic base case model, rock-fluid data resembling the Marcellus gas shale reservoir, the critical oil saturation to move is obtained to be \%35. As illustrated in Figure 30 for base case and following section maximum condensate saturation build up is around \%20 that implies this condensation does not move and results in formation damage and reducing well productivity.

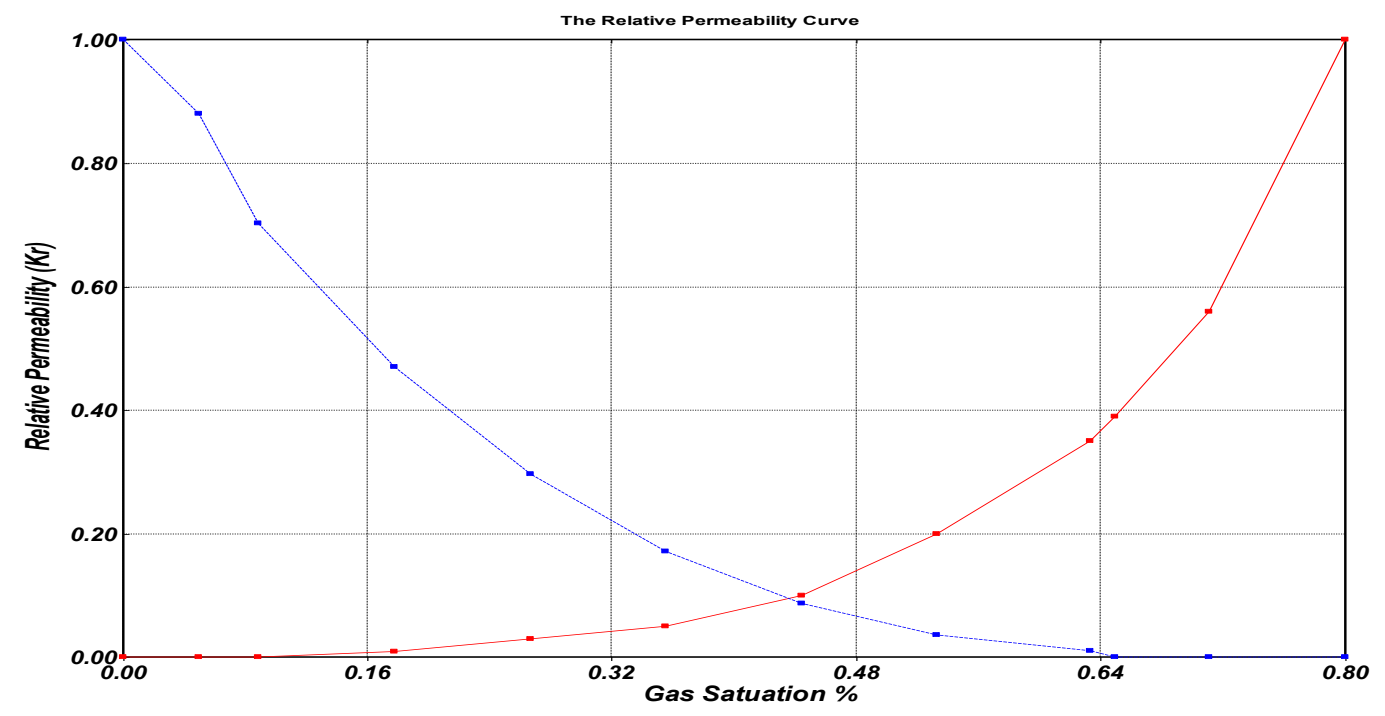

Figure 36: Rock-fluid graphical interpretation provided by the CMG simulator

Monitoring the condensation build up around a vertical well using base case conditions presented in Table 5 and Table 6 for the synthetic and actual reservoir models, demonstrated in figure 37, figure 38 and figure 39, a different condensation build up and saturation profiles have been observed. The condensate bank development in the cylindrical model figure 37 shows shorter damaged radius around the well in the long run (more than 10 years) due to the small drainage area around the well. In the case of actual Marcellus shale gas reservoir, well-2 was picked randomly to analyze the condensate bank development and saturation profiles around the well. In unconfined case (figure 38), where pore wall confinement is ignored saturation profiles show two 
distinct behavior sharp and smooth after short and long time production. If a $10 \%$ of the condensate saturation considered as an effective condensate saturation for reservoir damage, then the condensate bank radius is estimated to be $270 \mathrm{ft}$ around the well bore after 2 years, this radius extended to 900 feet considering long time production under dew point pressure of the reservoir. Considering same case including the pore wall confinement effects, Figure 39, and the delay of the liquid dropout was expected, due to lower dew point pressure. In this case unlike figure 38, figure 39 after two years of production no condensate build up has been observed. At later time also the maximum damage zone length was $1 / 3$ of case where the pore wall confinement effects ignored.

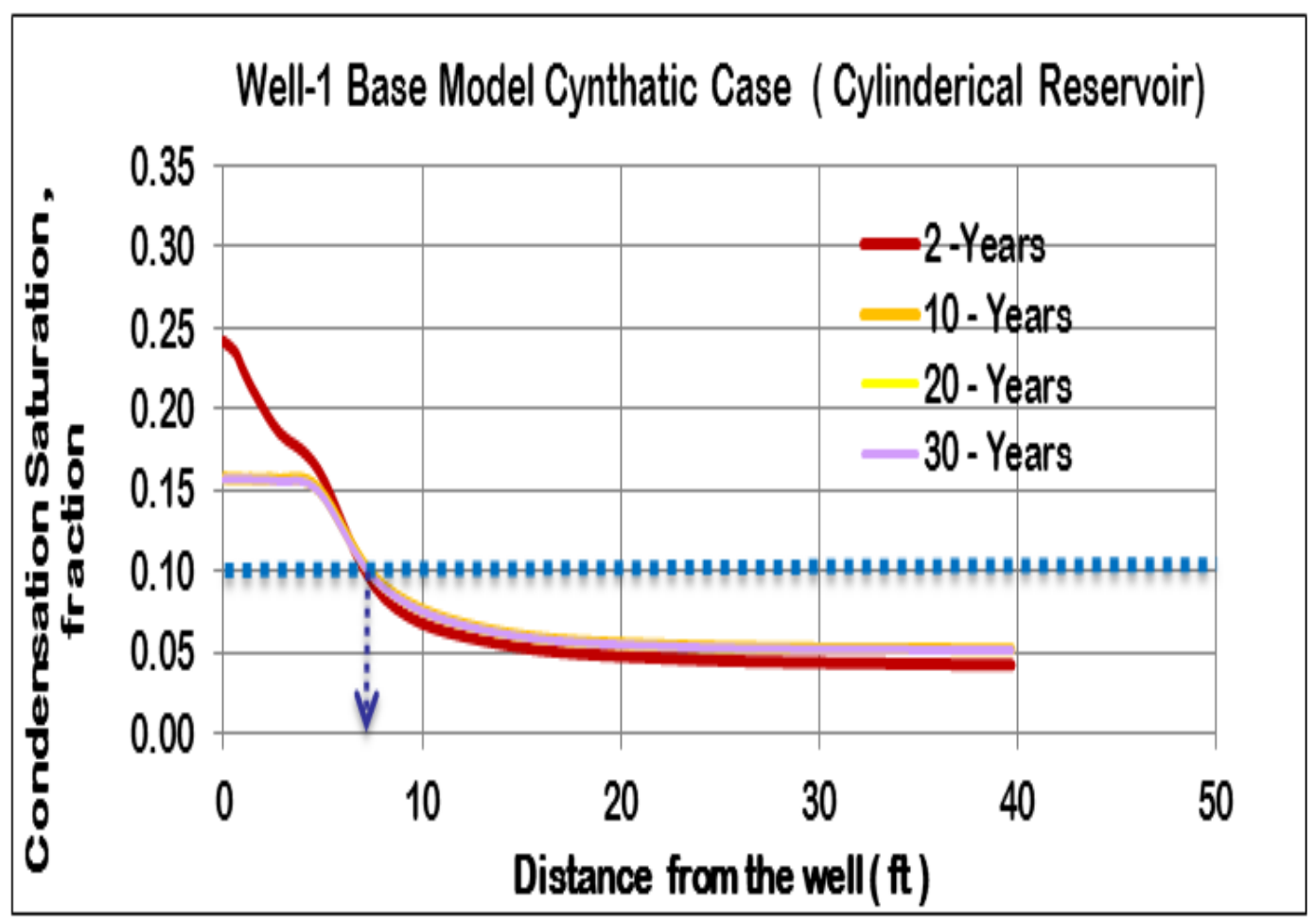

Figure 37: Condensation profile around well no. (1) Cylindrical Case. 


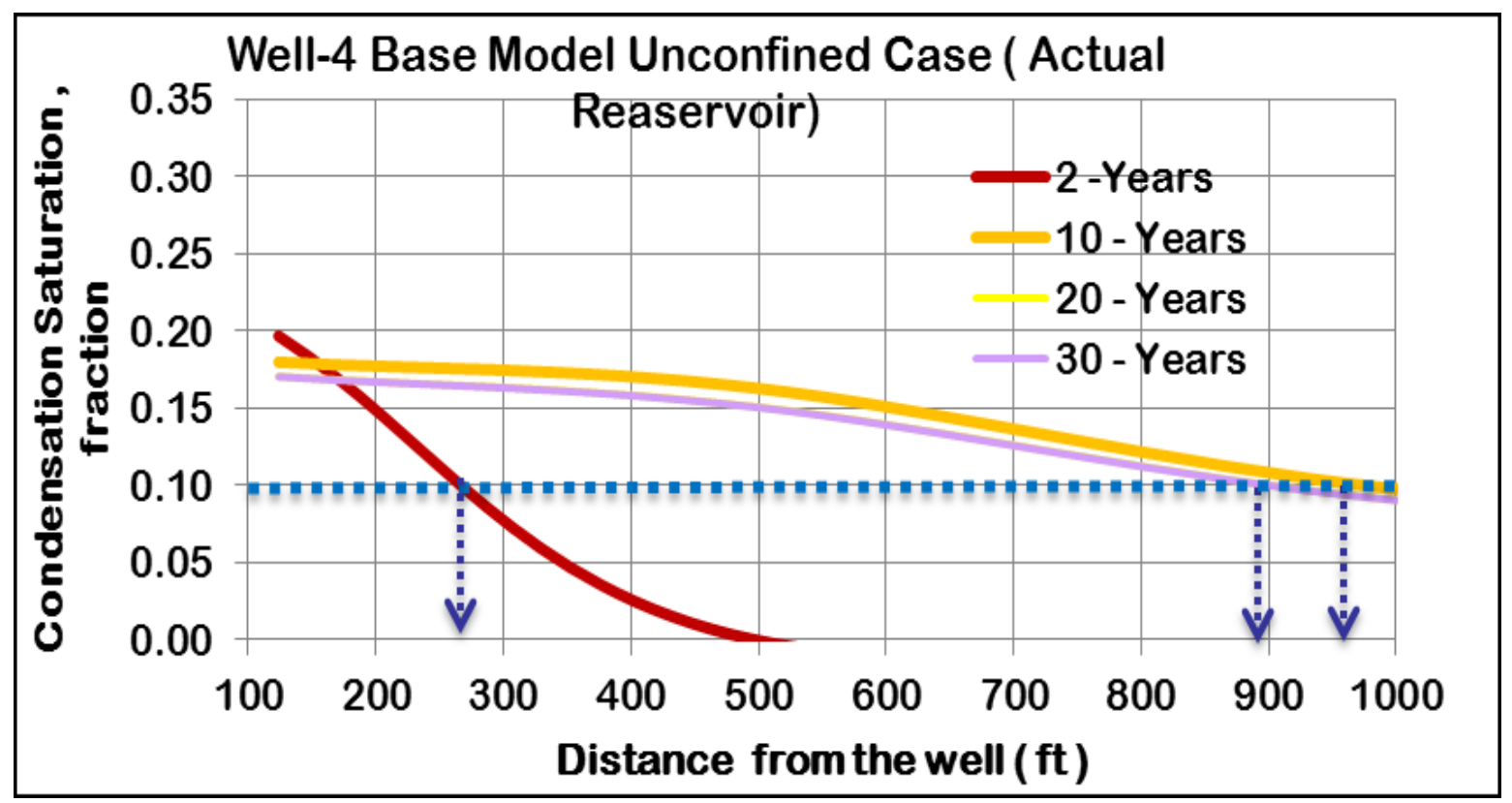

Figure 38: Condensation profile around well no. (2) Unconfined case.

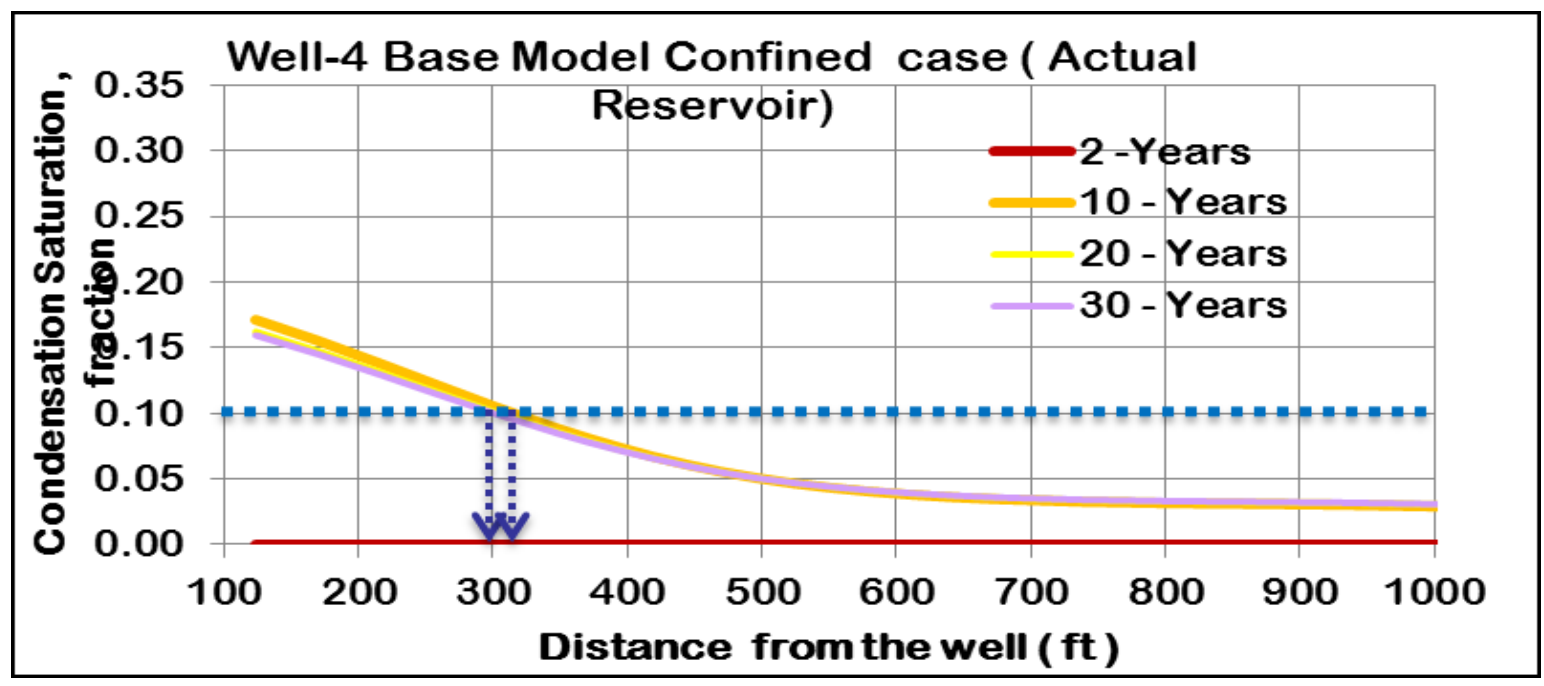

Figure 39: Condensation profile around well no. (4) Vertical well confined case.

Since the reservoir in my case is a rich gas shale formation, by considering the pore wall effect and the adsorbed phase density of the given gas mixture, the phase behavior of the fluid inside the multi-structure porous media was predicted as it is shown in figure 40. According to the operating condition presented in this figure, i.e., $\mathrm{FBHP}=1500$, the minimum pore sizes distribution that is affected by condensation is 6 
nano-meter. In addition to that the figure clearly shows the fluid behavior is not affected by the pore wall confinement in pores greater than 100 nano-meter.

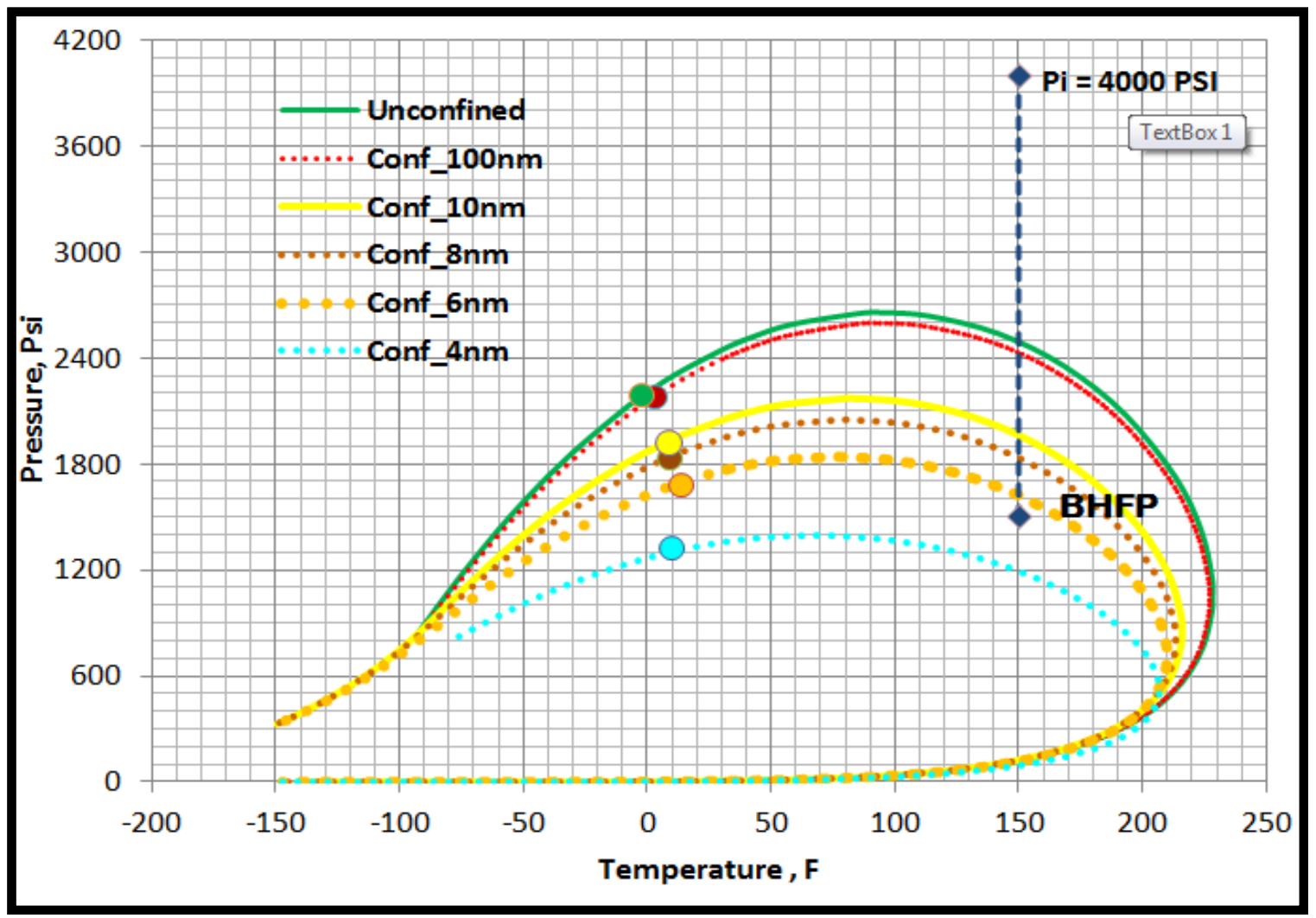

Figure 40: P-T phase diagram for different pore sizes

According to the operating condition decided for a reservoir depletion plan, the condition of the organic pores, whether they are filled with the condensate or not, can be foreseen at the depletion time as it can be seen in Figure 41. In different cases studied at this thesis, the average bottom hole flowing pressure was estimated to be 2300 Pisa based on average organic pore size typical of Marcellus that is $10 \mathrm{~nm}$. With this condition the condensation happens in pores larger than $10 \mathrm{~nm}$. This implies that we will not have any condensation in organic matters following the operation condition but we have in inorganics with larger average pore size more than $100 \mathrm{~nm}$. 


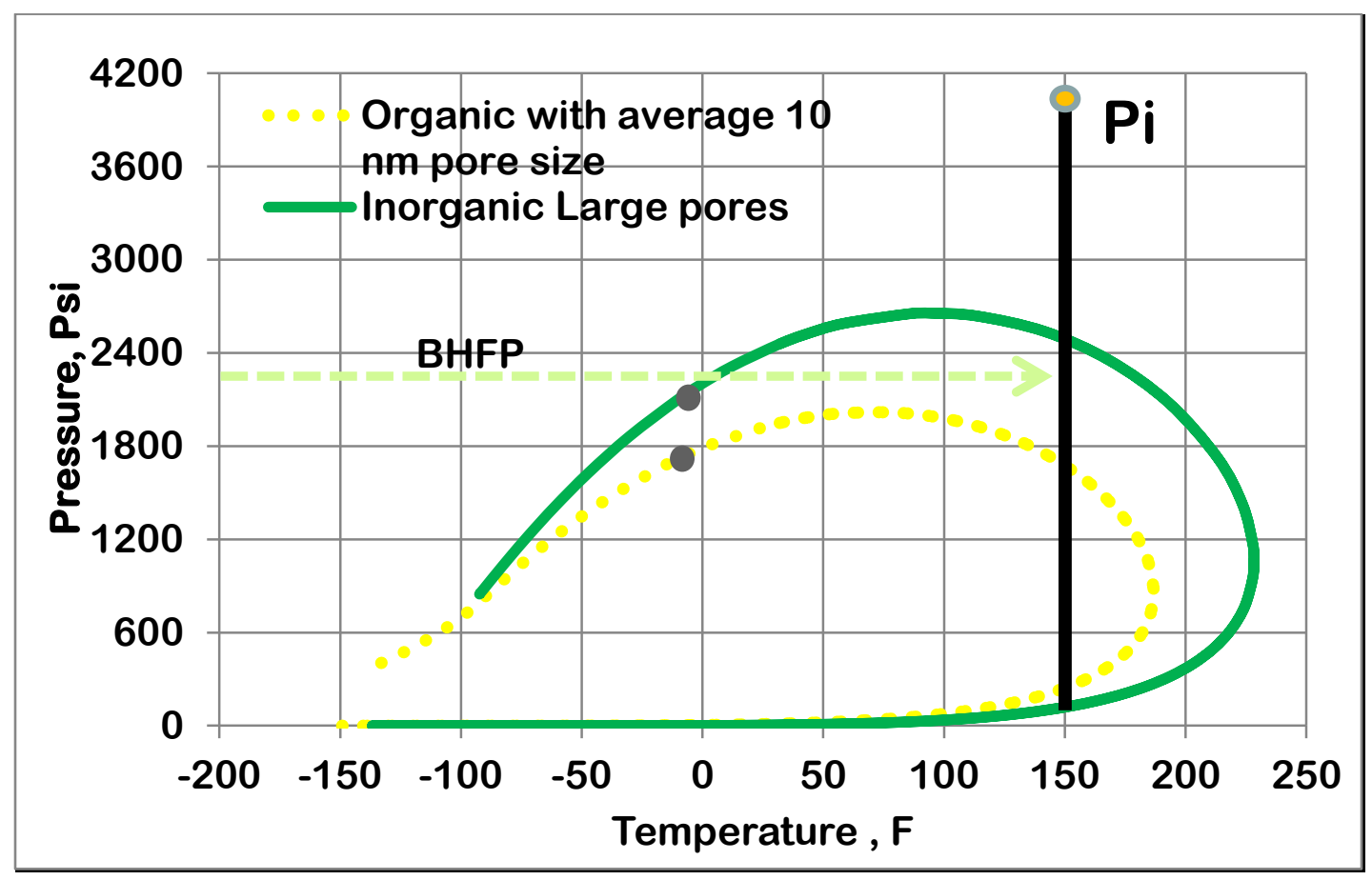

Figure 41: P-T Phase diagram illustrates condensate local influence by the operation condition.

The importance of the candidate parameters, Tables 8 and 9, studied here after pre and post screening were evaluated on Pareto chart as it is shown in Figure 42, Figure 43 and Figure 44. It clearly describes effects of parameters on the spread of the condensate ring around the producing wells. In the first graph, Figure 42, for the cylindrical case, porosity has minimal negative effect on the response, i.e., damaged zone radius. In Pareto chart Figure 42 left, porosity is shown as parameter $D$, that falls below the t-value implying that its effect is negligible on response. So, it was removed from the list of important parameters in the actual reservoir model. It also shows that composition-absolute permeability correlation has a negative impact on the response. That is interesting since increasing permeability leads to reducing the damaged zone radius however having rich fluid should increase the damaged zone radius. In this case the overall response of competing high permeability with rich composition is in favor of reducing the damaged zone radius. Similar analysis of Pareto charts have been performed for the cases considering (confined) and ignoring (unconfined) organic pore wall confinement effects Figure 43 and 44. In both (confined and unconfined) cases for the actual reservoir model, the increase in the rock compressibility, the hydraulic 
fracture spacing, the richness of the fluid composition and the pressure variation have different levels of a positive impact on the condensate spread inside the reservoir. The biggest impact is due to the increase in the rocks compressibility which serves the permeability reduction. This results in the pore volume reduction due to decrease in pore pressure and increasing the effective stress. The situation becomes even worse considering the adsorbed layer thickness. The hydraulic fracture spacing has also a considerable impact on the condensate build-up amount. The closer the fracture to each other the better, because it helps in producing the condensate thus reduces the damage zone around the wells. As for the fluid composition, the percentage of the heavy components of fluid is a direct indicator of the liquid dropout possibility. Lastly, less pressure differential between initial reservoir pressure and FBHP leads to average reservoir pressure higher than dew point therefore eliminates the condensation possibility or in case drops below the dew point leads less drop out of liquid. Also, with the confined case since the fluid phase envelope is smaller, the more time the reservoir pressure maintained above the dew point pressure the better chance to be given for the reservoir to produce heavy components. On the other hand, the increase in number of horizontal wells, the heterogeneity of the reservoir, and natural fractures density impact the spread of the condensate ring negatively. The increased number of the horizontal wells exposes a more pay zone area to production and thus accesses the condensate local areas. The natural fracture density creates a porous network service transporting the condensate towards producing wells. 


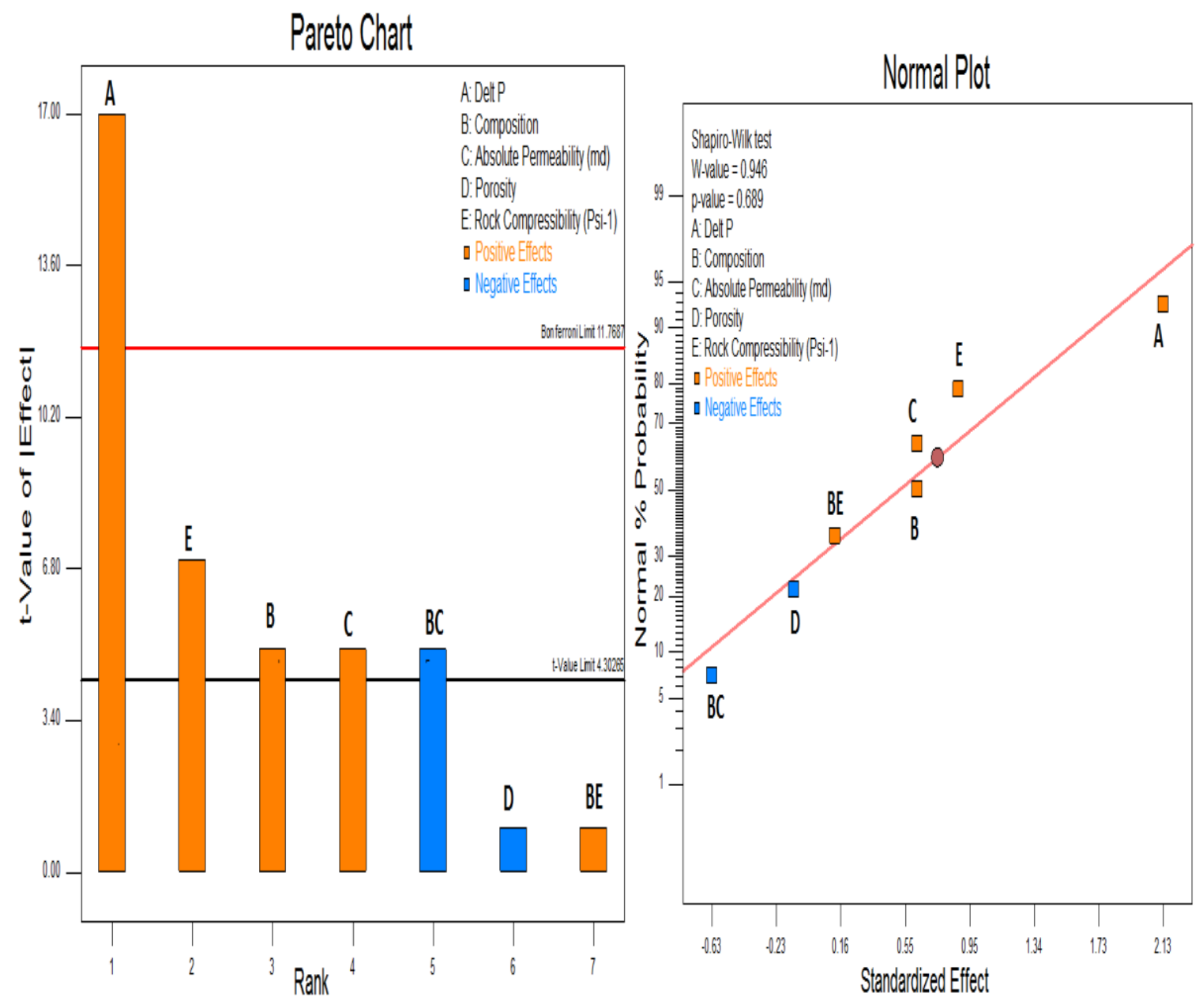

Figure 42: Analysis for 5 candidate parameters (Cylindrical Base Case Model) Design-Expert Software 

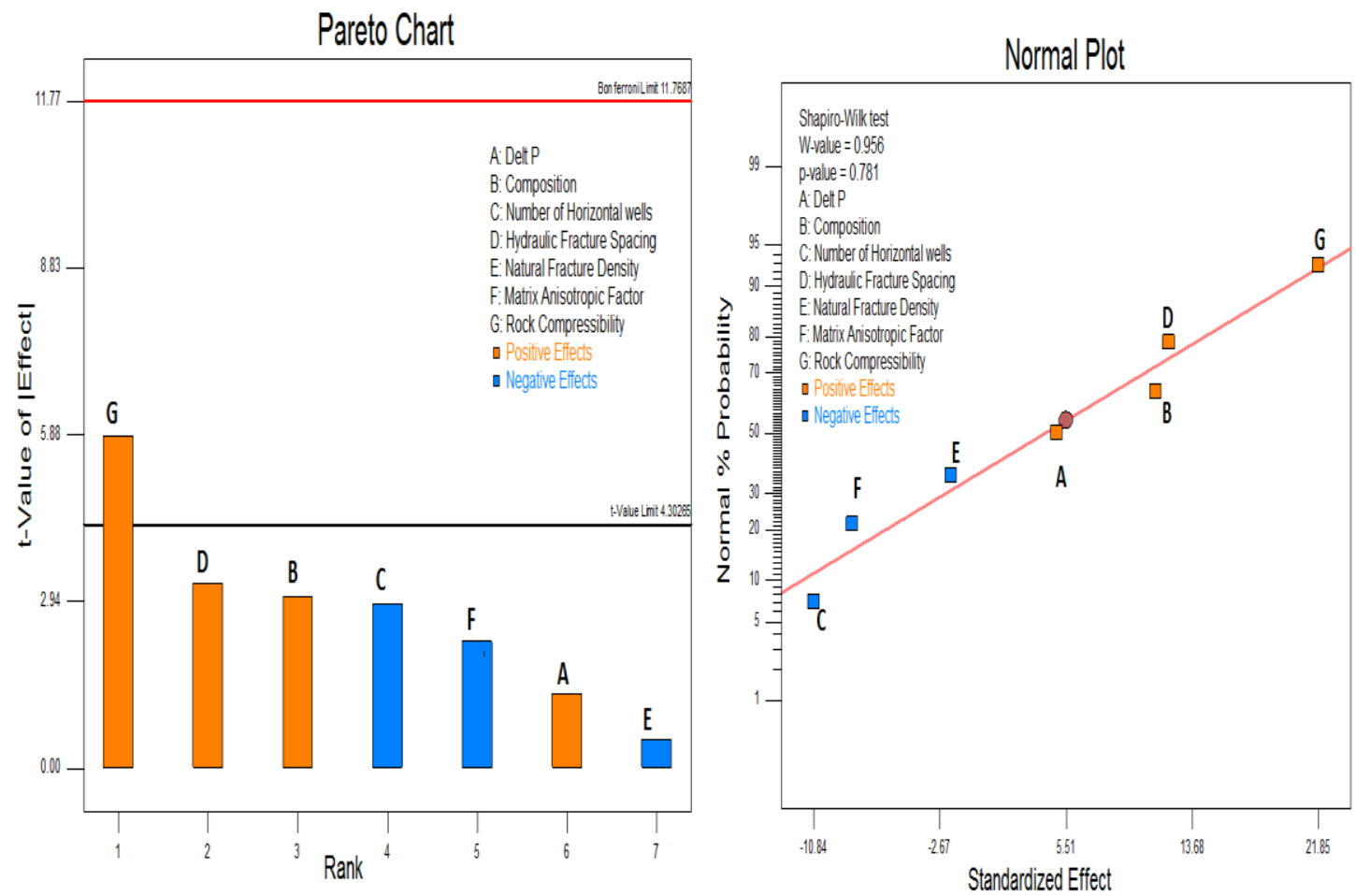

Figure 43: Analysis for 7 candidate parameters (unconfined Base Case Model) Design-Expert Software
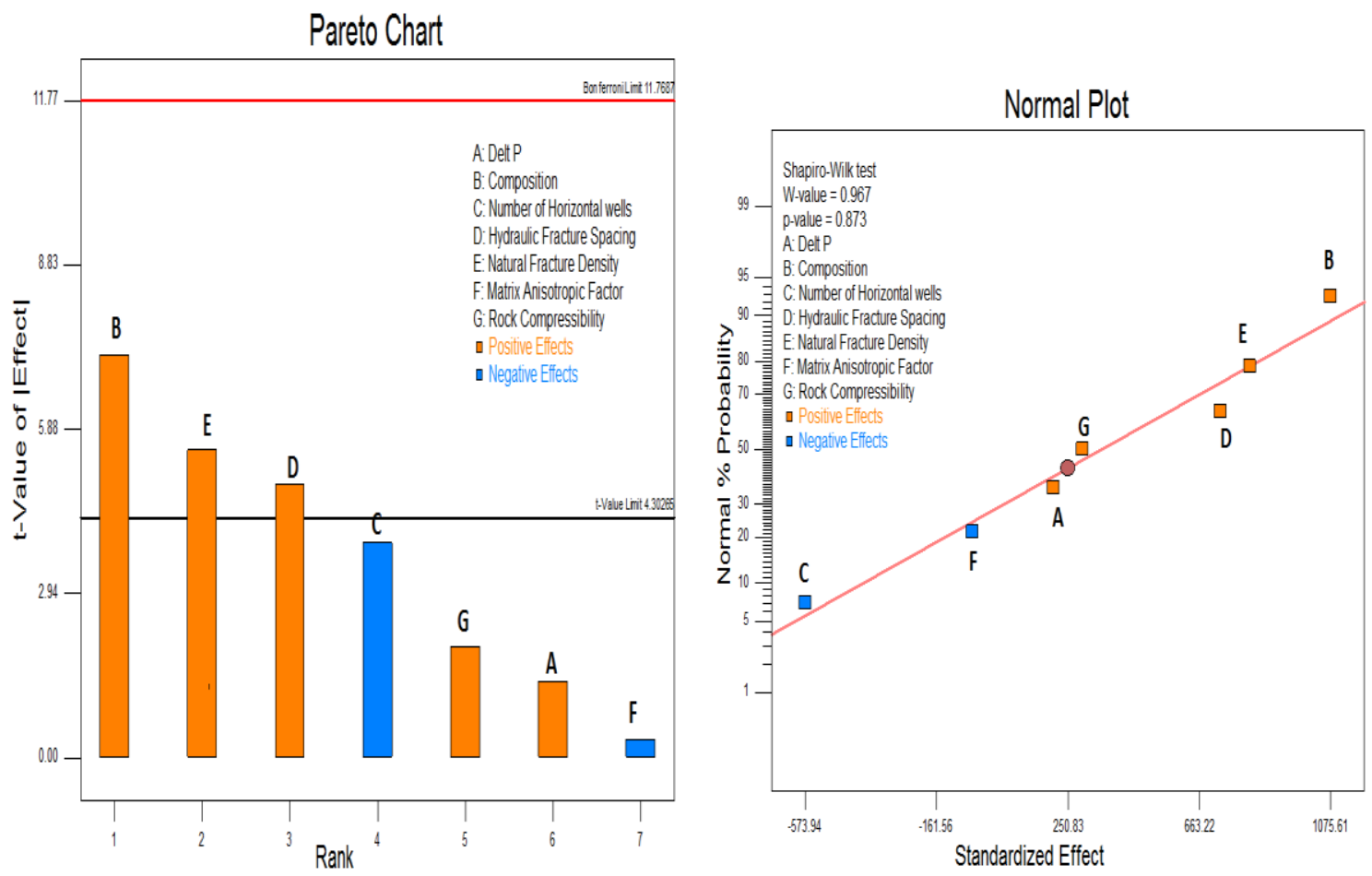

Figure 44: Analysis for 7 candidate parameters (confined Base Case Model) Design-Expert Software 
Figure 45 and 46 depicts the typical analysis of interactions between different parameters on the response of the reservoir. In these two figures, the interaction between number of horizontal wells and reservoir matrix anisotropy has been plotted. It is clear that at highest number of horizontal wells $(+1)$ and highest matrix anisotropy $(+1)$ for both cases of including and ignoring organic pore wall confinement effects, i.e., confined and unconfined, the damaged radius is minimal showing positive relation between these two parameters and their overall negative impact on damged zone extension. However, there is a distinct difference between these two cases and that can be seen clearly from surface responses in figures 45 and 46 right. In unconfined model anisotropy and number of horizontal wells seems to have similar impact on damaged radius, note that the counter lines of damaged radius with slope of $(-1)$ while in confined model impact of number of horizontal wells are significantly higher than anisotropy of the matrix that completely masks the anisotropy effect on damaged zone radius. Similar conclusion could also derived based on Figure 43 and 44 where columns $F$ and $C$ (matrix anisotropy and number of horizontal wells) have comparable importance in unconfined model while in confined model parameter " $C$ " has notably higher impact on damaged radius.
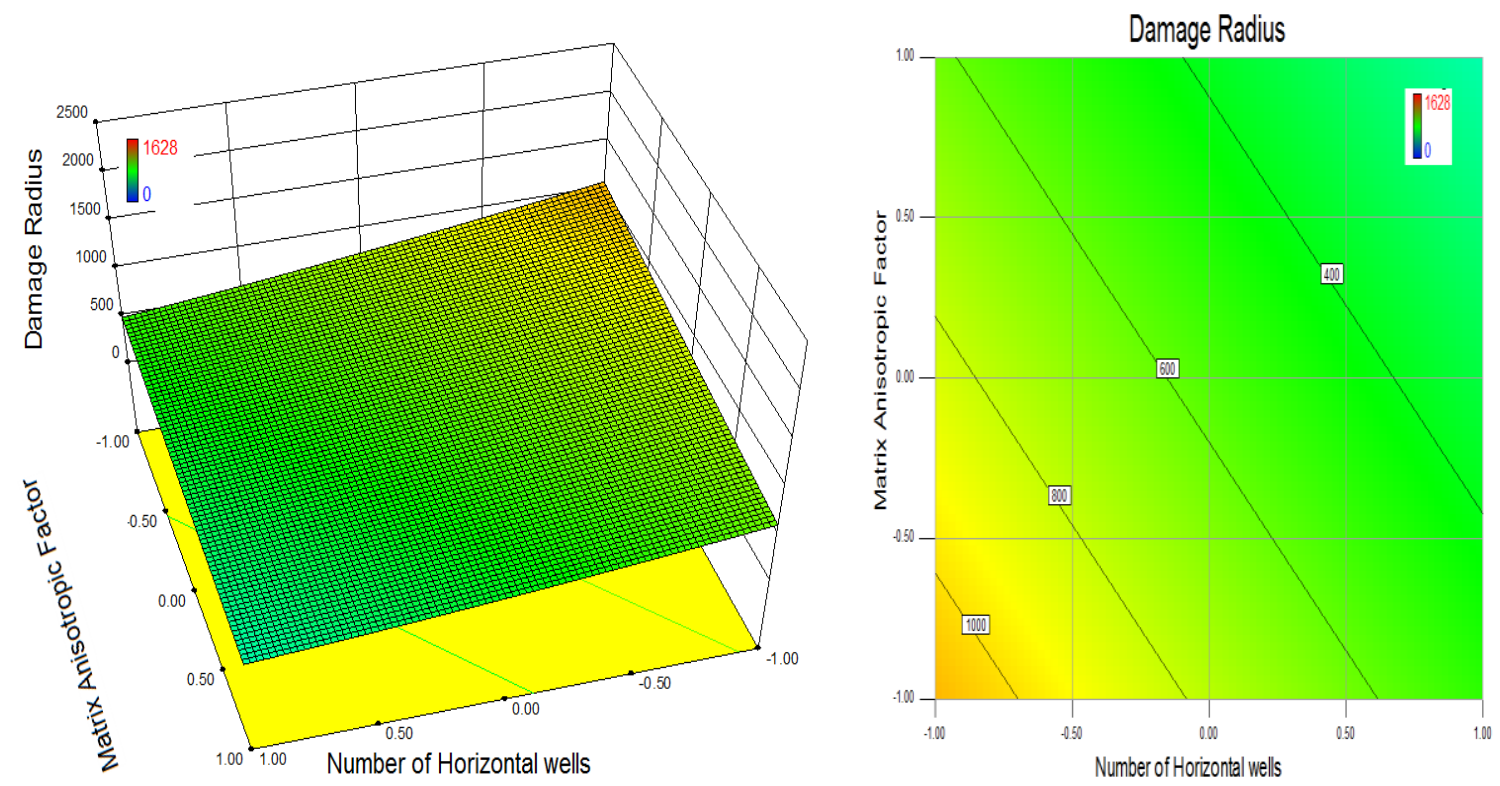

Figure 45: Interaction between parameters have the most negative impact on the condensate ring extension for unconfined case. 

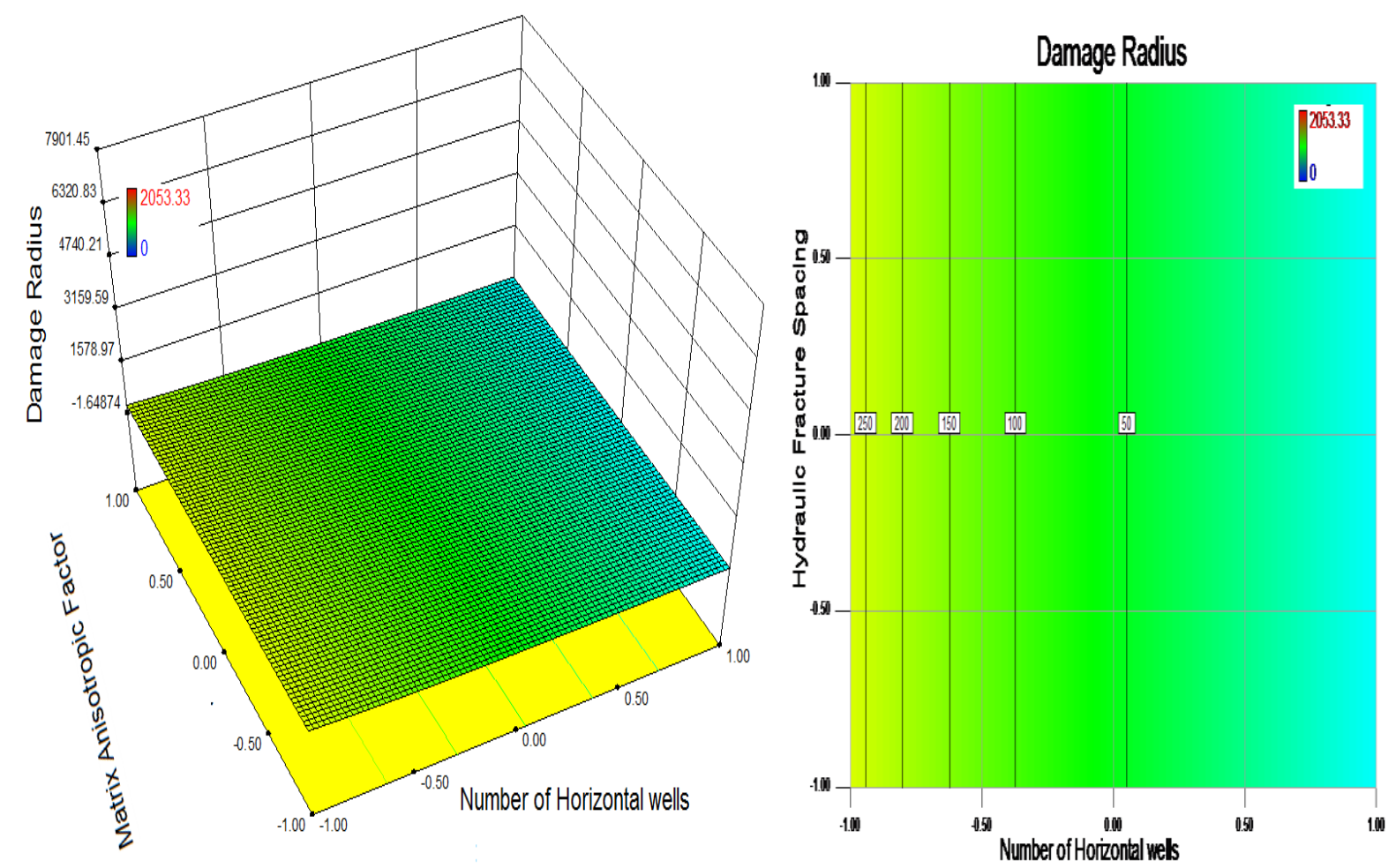

Figure 46: Interaction between parameters have the most negative impact on the condensate ring extension for confined case.

Figure 47 compares the gas production prediction of Marcellus shale gas reservoir for two base cases with consideration of organic pore wall confinements (confined) and ignoring that (unconfined). There are two major observations can be drawn from this figure, first both models predict the same gas production rates at early times where the pressure is above both dew points for these models. Later, as time passes pressure falls below the dew point for unconfined case since it has a larger envelope with higher dew point pressure that leads to starting the condensation, i.e. damaging the reservoir, therefore reducing the gas production rate in compare to confined case with much lower dew point. After 10 years reservoir pressure falls below dew point of confined model and damage zone starts developing in small pores reducing the production rate. Second observation is the ultimate cumulative gas production that is higher for the case ignoring the organic pore confinement effects. This is due to the fact 
that presence of pore wall confinements reduces the critical pressure and temperature of the reservoir fluid therefore increasing the gas compressibility factor and gas formation volume factor and reducing the gas viscosity. Changes in fluid properties due to wall confinement effects results in reduction in original gas in place calculation and therefore ultimate cumulative gas production. Behnaz 2012 using molecular simulation technique has reached to the similar conclusion.

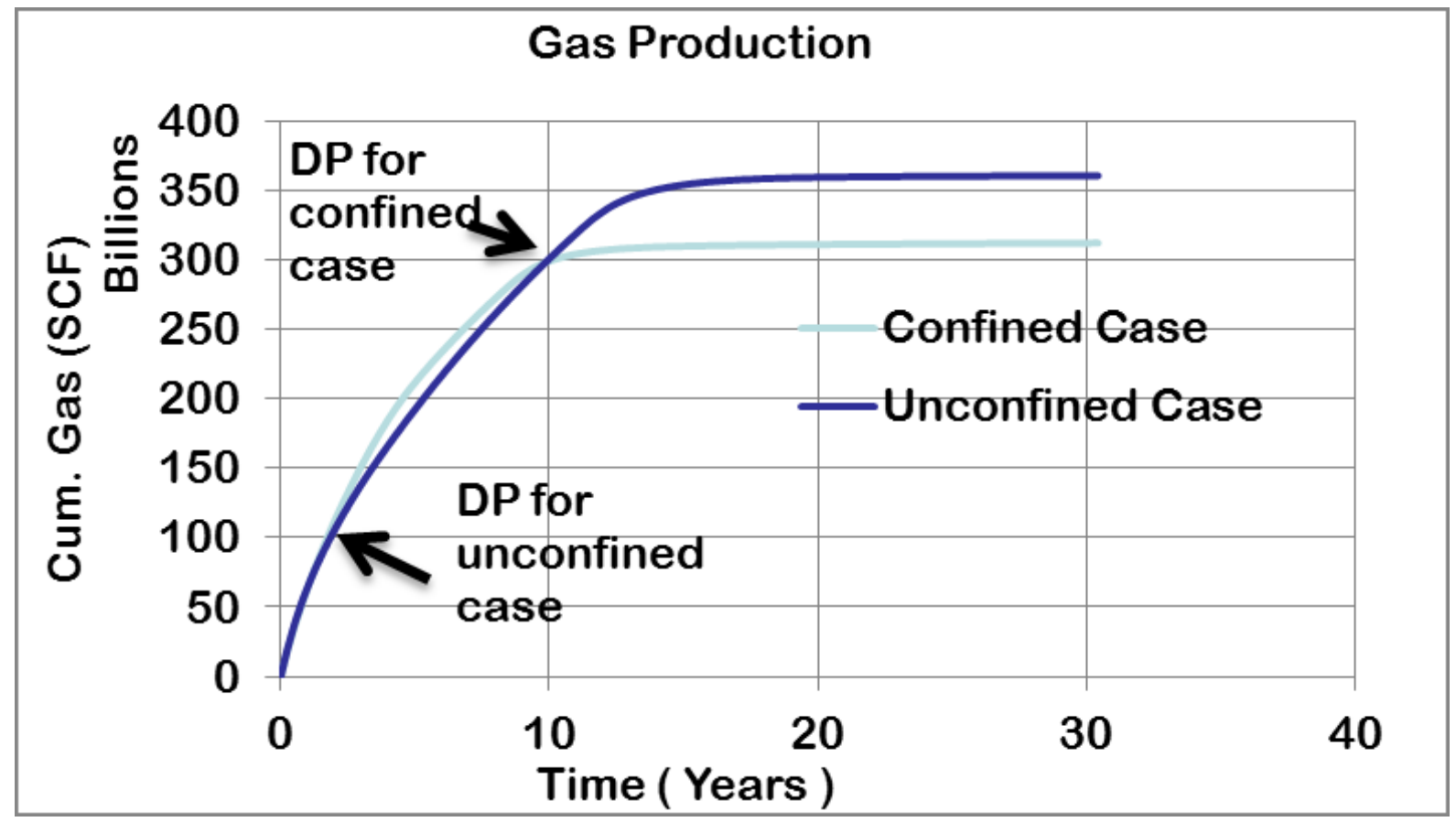

Figure 47 : Gas production for the base case model of the actual reservoir.

Compositional reservoir simulator (CMG-GEM) has been used through different runs to investigate the impact of Marcellus shale gas reservoir rock and fluid properties, operational conditions and also reservoir development plan, on condensate bank development and saturation dynamics. Based on the comparison of each run or confined and unconfined cases for the impact of different parameters on condensate bank developments are studied and presented in the Appendix A2(1-8), it is found that the mico-pore size characteristics of shale matrix can significantly alleviates the possible formation damage due to liquid drop out inside the reservoir. 


\section{2: Conclusions:}

- The methodology proposed in this work to obtain the thermodynamics of fluids confined in multi-scale pore structures such as shale gas reservoirs is illustrated to be an efficient way to describe the physics behind the behavior of fluids in organic rich shale reservoirs. In addition to that the key advantage of our approach is the fact that is very convenient for use in existing commercial simulators without the need for developing new simulators for shale gas reservoirs.

- The organic pores in the shale gas condensate reservoirs are vitally important because of their great contribution to the reservoir total pore volumes. It is our observation that reservoir fluid behaves differently under organic pore wall confinements due to pore wall confinement effect and also adsorbed layer effect. Change in reservoir fluid thermodynamics vanishes at pore sizes larger than 100 $\mathrm{nm}$ and becomes more pronounced at smaller pore sizes. The adsorbed phase density and adsorbed layer thickness effect is significant in critical properties of the fluid and also reserve estimation and production forecasts.

- The main conclusion that one can make out of this study is reservoir fluid phase envelope shrinks in nano-pores of organic rich shale reservoir. This leads to important conclusion that the condensation most probably happens in inorganic materials where the pore sizes are larger. For future work more detailed discussion in nano-scale using numerical technics such as Lattice Boltzmann or molecular dynamics is required to be able to accurately predict the adsorbed phase density and critical properties of different gas mixtures since the argument of homologous series in nano-pores needs to be validated. 


\section{REFERENCES:}

Adelola, G., Adesida, I., Yucel, A., Daniel, E., Resasco, \& Chandra, S. R. (2011, October). Characterization of Barnett shale pore size distribution using DFT analysis and Monte Carlo simulations. Paper presented at SPE Annual Technical Conference and Exhibition. Denver, CO. doi: 10.2118/147397-MS.

Akkutlu, I.Y., \& Ebrahim F. (2012). Multiscale gas transport in shales with local kerogen heterogeneities. SPE Journal, 17(4). doi: 10.2118/146422-PA.

Ambrose, R.J., Hartman, R.C., Mery, D., Yucel, A., \& Carl, H.S. (2010, February). New pore-scale considerations in shale gas in-place calculations. Paper presented at SPE Unconventional Gas Conference, Pittsburgh, PA. doi: 10.2118/131772-MS.

Arukhe, J. O., Aguilera, R., \& Harding, T. G. (2009, June). Dominant considerations for effective hydraulic fracturing in naturally fractured tight gas carbonates. Paper presented at Canadian International Petroleum Conference. Calgary, Alberta. doi: 10.2118/2009-153.

Behrenbruch, P., \& Goda H.M. (2006, September). Two-Phase Relative Permeability Prediction: A comparison of the modified Brooks-Corey methodology with a new Carman-Kozeny based flow formulation. Paper presented at SPE Asia Pacific Oil \& Gas Conference and Exhibition. Adelaide, Australia. doi:10.2118/101150-MS.

Bengherbia, M., \& Tiab, D. (2002, May). Gas-condensate well performance using compositional simulator: a case study. Paper presented at SPE Gas Technology Symposium Calgary Alberta. Calgary, Alberta. doi: 10.2118/75531-MS.

Bourbiaux B.J. (1994, October). Parametric study of gas-condensate reservoir behavior during depletion: a guide for development planning. Paper presented at European Petroleum Conference. London, United Kingdom. doi: 10.2118/28848-MS.

Cheng, Y. (2012). Impacts of the number of perforation clusters and cluster spacing on production performance of horizontal shale-gas wells. SPE Reservoir Evaluation \& Engineering journal. 15(1).31-40. doi: 10.2118/138843-PA.

Campos, M.D., Yucel, A., \& Sigal, R.F. (2009, October). A molecular dynamics study on natural gas solubility enhancement in water confined to small pores. Paper presented at SPE Annual Technical Conference and Exhibition. New Orleans, LA. doi: 10.2118/124491-MS.

Curtis. H, Whitson, Pera, F. \& Aud, S. (1999, October). Gas condensate relative permeability for well calculation. Paper presented at SPE Deep Gas Conference and Exhibition. Houston. TX. doi: 10.2118/56476-MS. 
Curtis, M.E., Ambrose, R.J., Sondergeld, C.H., \& Rai, C.S. (2010, October). Structural characterization of gas shales on the micro- and nano-scales. Paper presented at Canadian Unconventional Resources and International Petroleum Conference. Calgary, Alberta. doi: 10.2118/165870-MS.

Design-Expert (Version 8). [Computer software manual]. Minneapolis, MN: Stat-Ease.

Devegowda, D., Sapmanee, K., Civan, F., \& Sigal, R. (2012, October). Phase behavior of gas condensates in shales due to pore proximity effects: Implications for transport, reserves and well productivity. Paper presented at SPE Annual Technical Conference and Exhibition. San Antonio, TX. doi: 10.2118/160099-MS.

Dubinin, M.M. (1960). The potential theory of adsorption of gases and vapors for adsorbents with energetically non-uniform surfaces. Chemical Reviews. 60(2). 235-241. doi: 10.1021/cr60204a006

Energy Information Administartion. (2011, July 6). Review of emerging resources: U.S. shale gas and shale oil plays. Retrieved from http://www.eia.gov/analysis/studies/usshalegas/pdf/usshaleplays.pdf.

Fan, L., Billy, W.H., A. Jamaluddin, A., Jairam, K., Robert, M., \& Gary, A.P., Alexander, S, \& Curtis H. W. (2005). Oilfield Review. Understanding gas condensate reservoirs. Retrieved from http://www.slb.com/ /media/Files/resources/oilfield_review/ors05/win05/02_under standing_gas_condensate.pdf. Accessed Sep. $201 \overline{3}$.

Fevang, O., \& Whitson, C. (1996). Modeling gas-condensate well deliverability. Spe Reservoir Engineering. 11(4). 221-230. doi: 10.2118/30714-PA.

Gelb, L.D., Gubbins, K.E., Radhakrishnan, R., Sliwinska, M. (1999). Phase separation in confined systems. Reports on Progress in Physics. 62(12).1573. doi:10.1088/0034-4885/62/12/201.

Geology. (2011, May 23). Marcellus shale - appalachian basin natural gas play new research results surprise everyone on the potential of this well-known devonian black shale. Retrieved from http://geology.com/articles/marcellus-shale.shtml.

Guillermo J., Zarragoicoechea, \& Victor A. K. (2002). Vander Waals equation of state for a fluid in a nanopore. Physical Review E. 65(2). doi: 10.1103/PhysRevE.65.021110.

Hall, K.R., Gustavo, A. \& Mansoori, G.A. (1993). Quadratic mixing rules for equations of state. Origins and relationships to the virial expansion. Fluid Phase Equilibria. 91. 67-76. 
Imo-Jack, O. (2010, August). PVT characterization of a gas condensate reservoir and investigation of factors affecting deliverability. Paper presented at Nigeria Annual International Conference and Exhibition. Calabar, Nigeria. doi: 10.2118/140629MS.

Jiang, J., Sanley, I.S., Merijn, S., \& Berend, S. (2005). Adsorption and separation of linear and branched Alkanes on Carbon nano-tube bundles from configurationalbias Monte Carlo simulation. Physical Review B. 72(4). doi:10.1103/PhysRevB.72.045447.

Jossi, J.A., Leonard, I.S., \& George, T. (2004). The viscosity of pure substances in the dense gaseous and liquid phases. AIChE Journal. 8 (1). 59-63. doi: 10.1002/aic.690080116.

Kang, S.M., Ebrahim, F., Ray, A.J., Yucel, A., \& Sigal, R.F. (2011). Carbon dioxide storage capacity of organic-rich shales. SPE Journal. 16(4). 842-855. doi: 10.2118/134583-PA.

Kim, B.H., Gyeong-Ho, K., \& Yamg-Gon, S. (2003). Adsorption of Methane and Ethane into single-walled Carbon nano-tubes and slit-shaped carbonaceous pores. Korean Journal of Chemical Engineering. 20(1). 104-109. doi: 10.1007/BF02697193

Kniazeff, V.J., Nvaille, S. A. (1965). Two-phase flow of volatile hydrocarbons. SPE Journal. 5(1). 37-44. doi: 10.2118/962-PA.

Loucks, R.G., Robert, M.R., Stephen, C. R., \& Daniel, M. J. (2009). Morphology, genesis, and distribution of nanometer-scale pores in siliceous mudstones of the Mississippian Barnett shale. Journal of Sedimentary Research. 79. 848-861. doi: 10.2110/jsr.2009.092.

Marcellus Shale. (2012, November 01) . Ultra petroleum updates Marcellus shale activity for third quarter of 2012. Retrieved from http://shale.typepad.com/marcellusshale/.

McCain W.D. (1990). The properties of petroleum fluids. Tulsa, OK .PennWell Publishing Company.

McDonald, I.R., (1972). NPT-ensemble Monte Carlo calculations for binary liquid Mixtures", Molecular Physics. 23(1). 41-58.

Miskimins, J. L., Lopez-Hernandez, H.D., Barree, R.D., \& Assocs. (2005, October). Nondarcy flow in hydraulic fractures: does it really Matter?. Paper presented at SPE Annual Technical Conference and Exhibition. Dallas , TX. doi: 10.2118/96389MS. 
Mott, R., Andrew, C., \& Mike, S. (1999, October). A New Method of Measuring Relative Permeabilities for Calculating Gas-Condensate Well Deliverability. Paper presented at SPE Annual Technical Conference and Exhibition. Houston, TX. Doi: 10.2118/56484-MS.

Moncrieff, J., Sondergeld, C., Ambrose, R., \& Rai, C. (2010, February). Micro-structural studies of gas shales. Paper presented at SPE Unconventional Gas Conference. Pittsburgh, PA. doi: 10.2118/131771-MS.

Morishige, K., Fujii, H., Uga, M., \& Kinukawa, D. (1997). Capillary critical point of Argon, Nitrogen, Oxygen, Ethylene, and Carbon Dioxide in MCM-41. American Chemical Society. 13 (13) . 3494-3498. doi: 10.1021/la970079u.

Morishige, K. \& Shikimi, M. (1998). Adsorption hysteresis and pore critical temperature in a single cylindrical pore. Journal of Chemical Physics. 108 (18). 7821-7824.

Muskat, M. \& Meres, M.W. (1936). The flow of heterogeneous fluid through porous media. Jurnal of applied physics. 7(9). 346-363.

Orangi, A., Nagarajan, N. R., Honarpour, M. M., \& Rosenzweig, J. (2011, January). Unconventional shale oil and gas-condensate reservoir production, impact of rock, fluid, and hydraulic fractures. Paper presented at SPE Hydraulic Fracturing Technology Conference. The Woodlands, TX. doi: 10.2118/140536-MS.

Ortiz, V., Lopez Y.M. \& Lopez, G.E. (2005). Phase diagrams and capillarity condensation of methane confined in single- and multi-layer nanotubes. Molecular Physics. 103 (19). 2587-2592.

Panagiotopoulos, A.Z., (1987). Direct determination of phase coexistence properties of fluids by monte carlo simulation in a new ensemble. Molecular Physics. 61(4). 813-826.

Peterson, B.K., Keith,E., Gubbins, G.S., Heffelfinger, U. M., Bettolo, M., \& Frank, V. S. (1988). Lennard-Jones fluids in cylindrical pores: Nonlocal theory and computer simulation. Journal of Chemical Physics. 88 (10). doi: 6487-6500.

DOI:10.1063/1.454434.

Pope G.A., Wu, W., Narayanaswamy, G., Delshad, M., Sharma, M., \& Wang, P. (1998). Modeling relative permeability effects in gas-condensate reservoirs. Department of Petroleum and Geosystems Engineering. doi: 10.2118/49266-MS.

Qi, Z., Sinopec, Z., Baosheng, L., Ruijian, D., Zhimin, Du., Shouping, W. \& Wei, Z. (2007, June). Phase behavior study in the deep gas-condensate reservoir with low permeability. Paper presented at Europec/Eage Conference and Exhibition. London, U.K. doi: 10.2118/107315-MS. 
Rahmani, B. D. (2012). Multi-component shale gas in-place calculations. (Master's thesis), University of Oklahoma, Norman, OK.

Roth, M. (2013). Gas shale reservoirs- similar, yet so different. Retrieved from https://www.transformsw.com/wp-content/uploads/2013/05/Shale-Gas-ReservoirsSimilar-yet-so-different-2010-RMAG-DGS-3D-Symposium-Roth.pdf.

Rowley, L.A., Nicholson, D., \& Parsonage, N.G., (1975). Monte Carlo grand canonical ensemble calculation in a gas-liquid transition region for 12-6 Argon. Journal of Computational physics. 17(4). 401-414.

Sapmanee, K. (2011). Effect of pore proximity on behavior and production of Gas/ Condensate. (Master's thesis). University of Oklahoma. Norman, OK.

Singh, S.K., A., Deo, G. and Sing, J.K. (2009). Vapor-liquid phase coexistence, Critical properties, and surface tension of confined alkanes. Journal of physical chemistry C. 113(17). 7170-7180.

Sondergeld, C. H., Newsham, K. E., Comisky, T. E., Rice, M. C. \& Rai, C. S. (2010, February). Petrophysical considerations in evaluating and producing shale gas resources. Paper presented at SPE Unconventional Gas Conference. Pittsburgh, PA. doi: 10.2118/131768-MS.

Spivak, A. (1974). Gravity segregation in two-phase displacement process. SPE Journal, 14(6). doi:10.2118/4630-PA.

Standing, M. B., \& Donald, K. L. (1942): Density of natural gases. Transactions of the AIME. 146(1). 140-149. doi: 10.2118/942140-G.

Sudhir, K.S., Ankit S., Goutam, D., \& Jayant, K. S. (2009). Vapor-liquid phase coexistence, critical properties, and surface tension of confined alkanes. Journal of physical chemistry C. 113(17). 7170-7180.

Wang, F. P., \& Reed, R. M. (2009, October). Pore Networks and Fluid Flow in Gas Shales. Paper presented at SPE Annual Technical Conference and Exhibition. New Orleans, LA. doi: 10.2118/124253-MS.

Wheaton, R. J., \& Zhang, H. R. (2000, October). Condensate banking dynamics in gas condensate fields: compositional changes and condensate accumulation around production wells. Paper presented at SPE Annual Technical Conference and Exhibition. Dallas, TX. doi: 10.2118/62930-MS. 
Whitson, C.H., \& Sunjerga, S. (2012, October). PVT in liquid-rich shale reservoirs. Paper presented at SPE Annual Technical Conference and Exhibition. San Antonio, TX. doi: 10.2118/155499-MS.

Yuan, P. and Laura, S. (2006). Equations of state in a lattice Boltzmann model. Physics of Fluids. 18(4).

Zarragoicechean G.J., and Kuz, V.A. (2004). Critical shift of confined fluid in a nanopore. Fluid Phase Equilibria. 220(1).7-9.

Zhilin, Qi., Baosheng, L., Ruijian, D., Zhimin, D., Zhimin, W., \& Wei, Z. (2007, June). Phase Behavior Study in the Deep Gas-Condensate Reservoir with Low Permeability. Paper presented at Europec/Eage Conference and Exhibition. London, U.K. doi: 10.2118/107315-MS. 


\section{APPENDIX}

\section{Appendix A: The CMG Software Application:}

\section{A1: The CMG Software Inputs:}

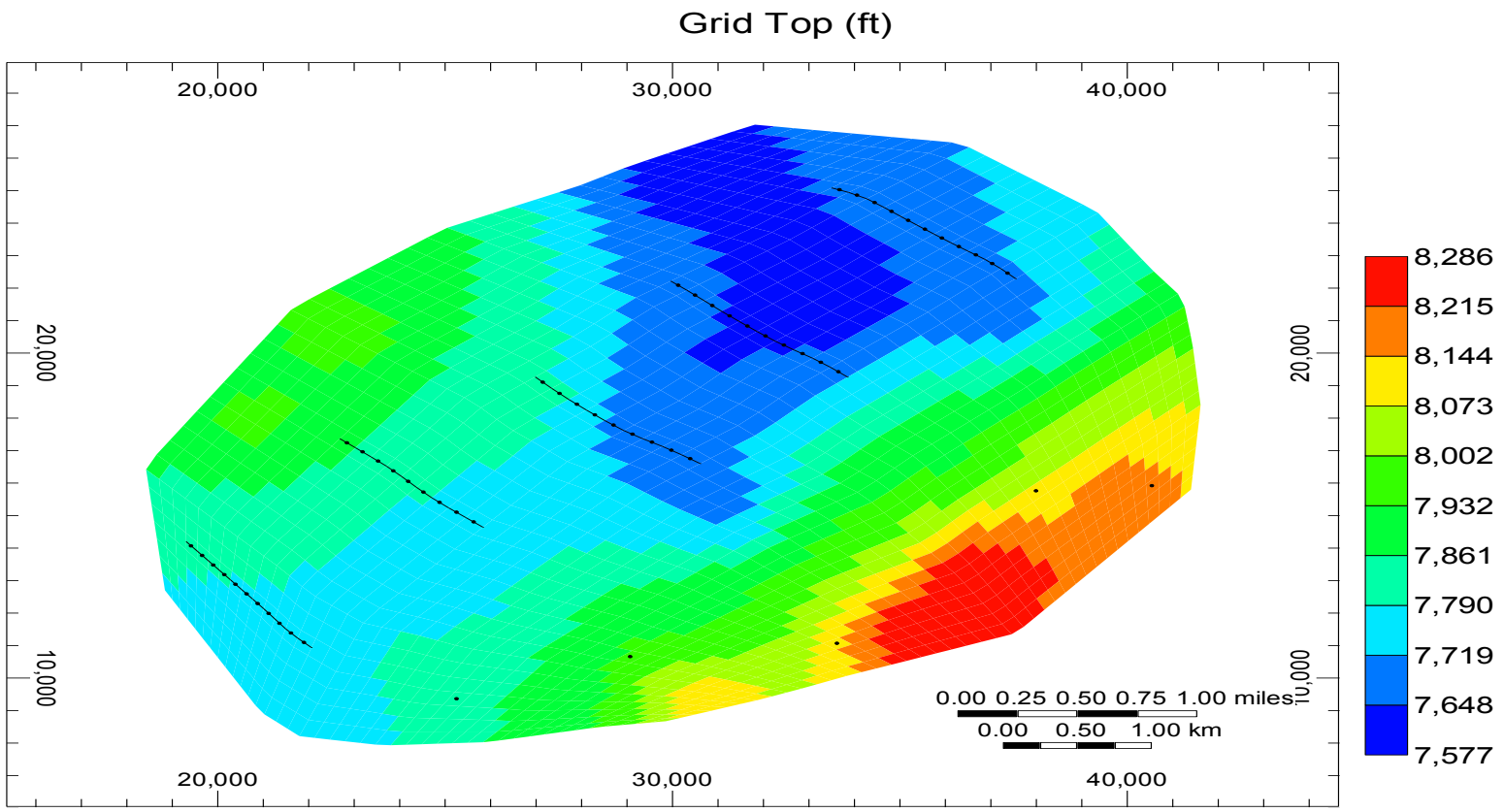

Figure (A1. 1 ): Marcellus structure map.

Grid Thickness (ft)

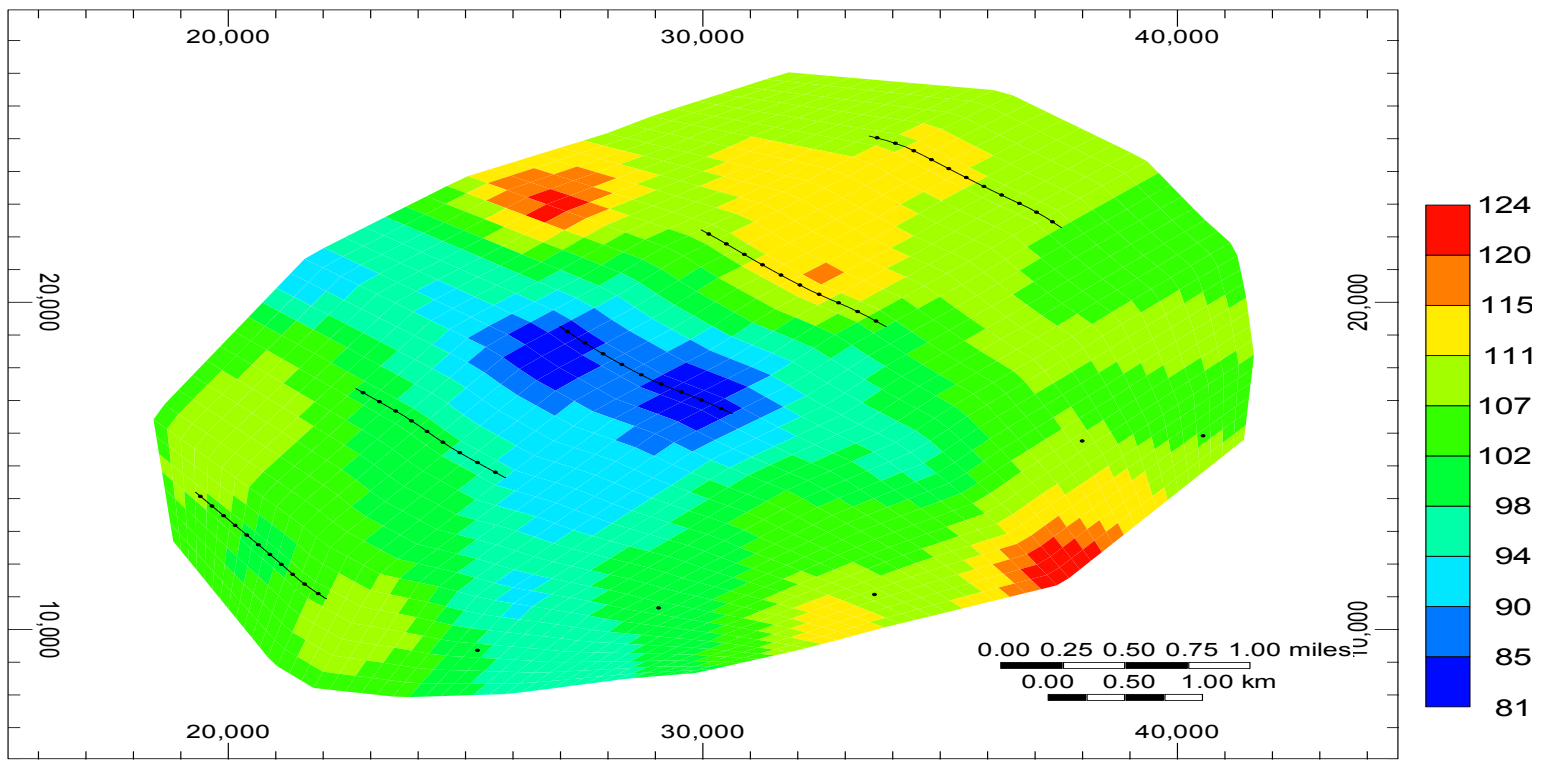

Figure (A1. 2): Marcellus thickness map. 


\section{Permeability I (md)}

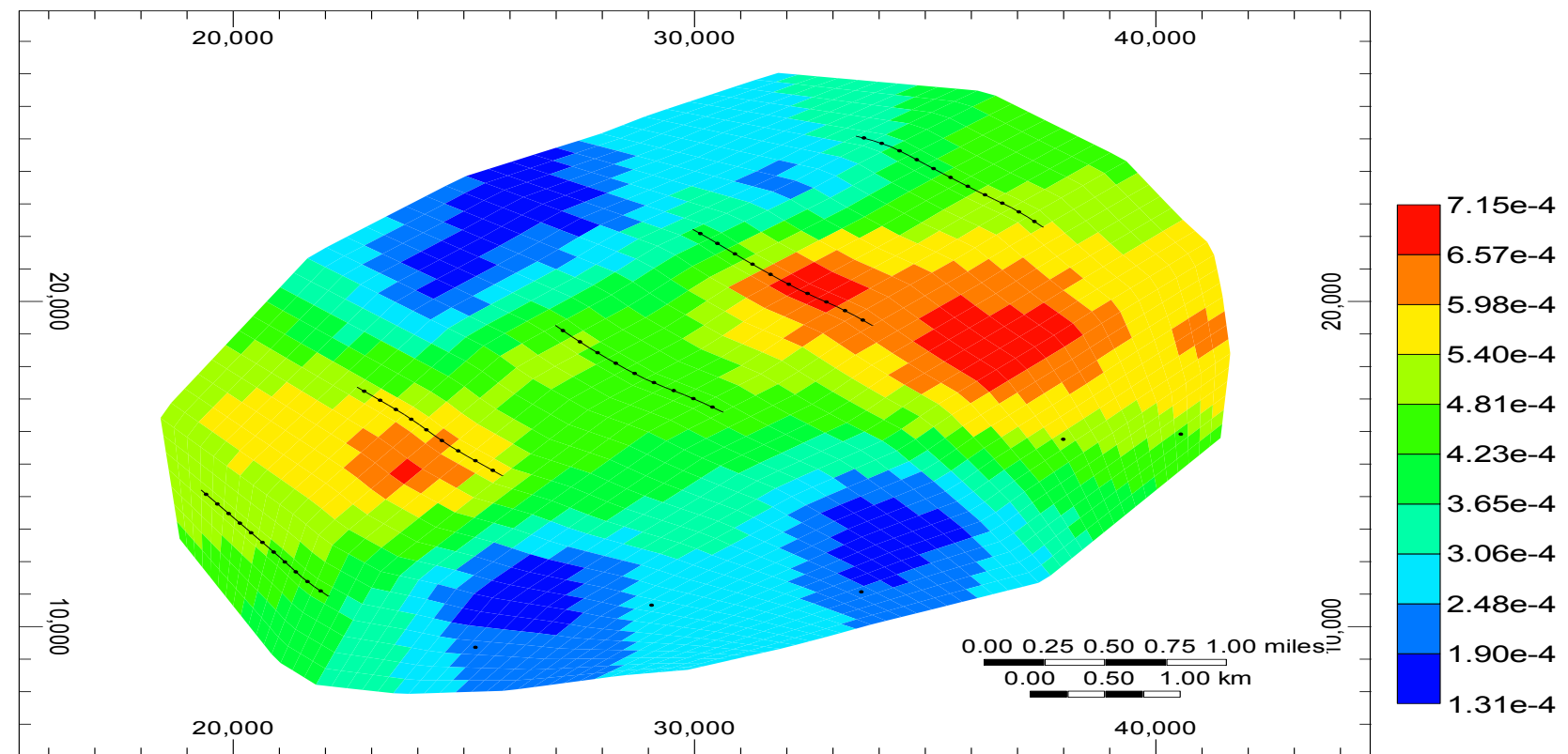

Figure (A1. 3): Marcellus permeability map.

\section{Porosity ( fraction)}

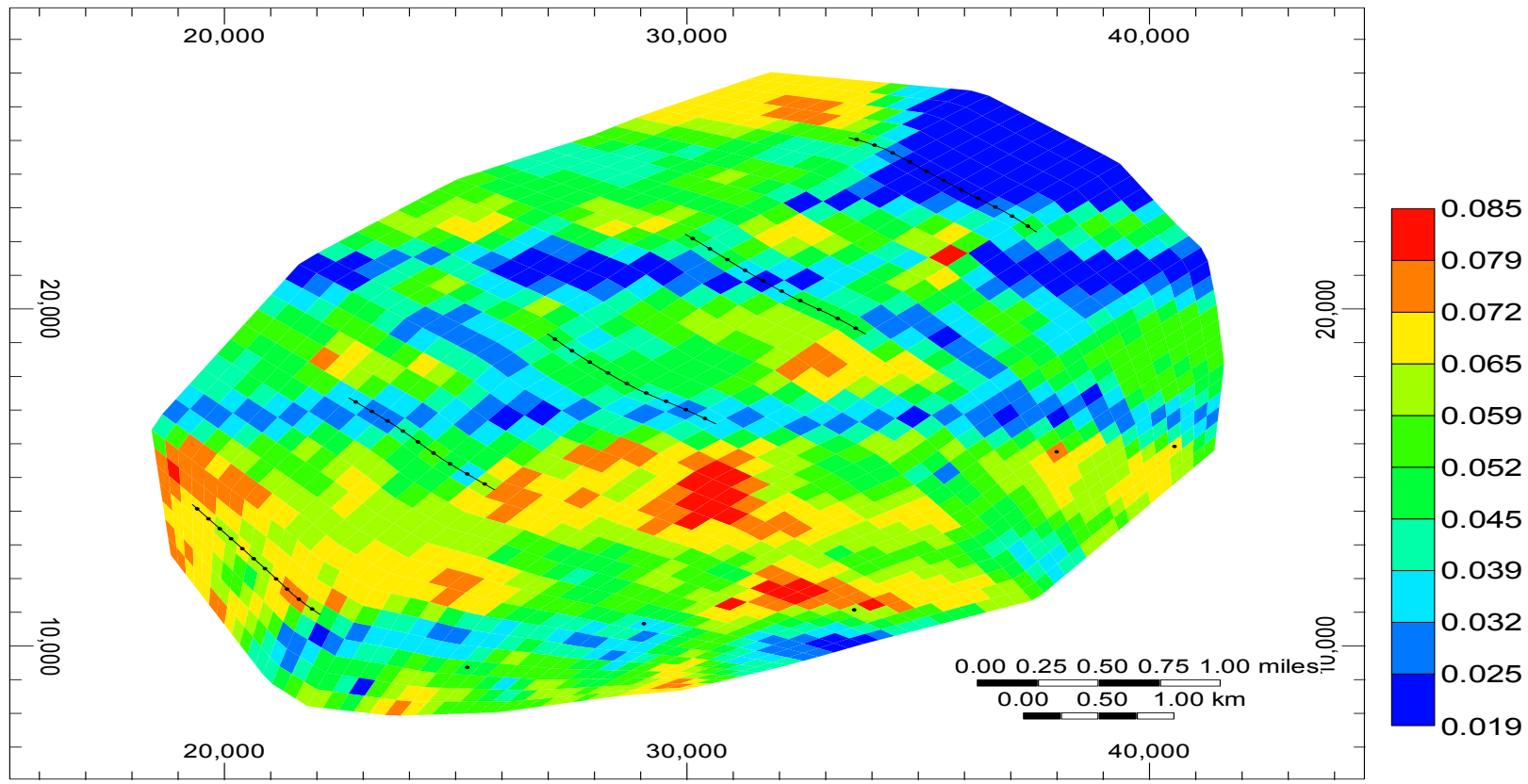

Figure (A1. 4): Marcellus porosity map. 


\section{A.2: Detailed investigation of condensation build up around different wells with and without pore wall confinement effects}

Comparison between two cases for Run 1 (presented in table 10) clearly shows that considering organic pore wall confinements that shrinks the phase envelope completely removes the possibility of having condensate development in the reservoir matrix while using conventional approach predicts damage zone around most of the wells.

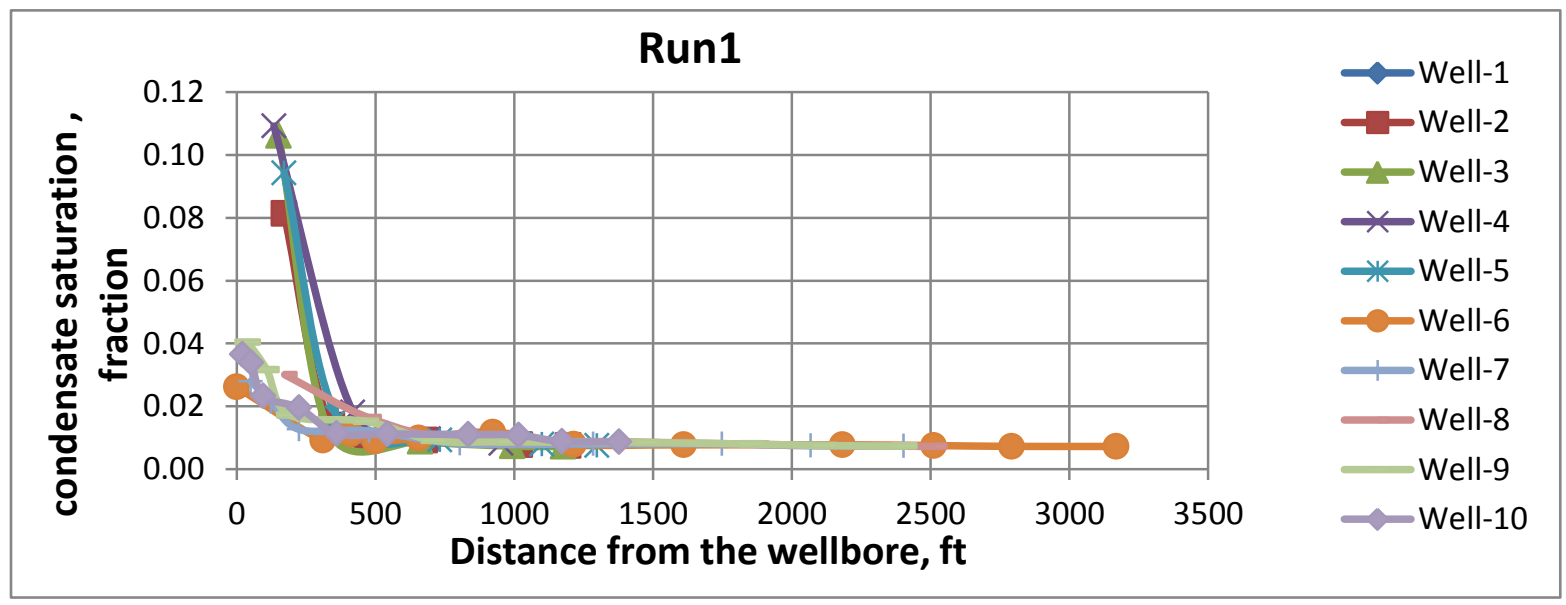

a) : unconfined case.

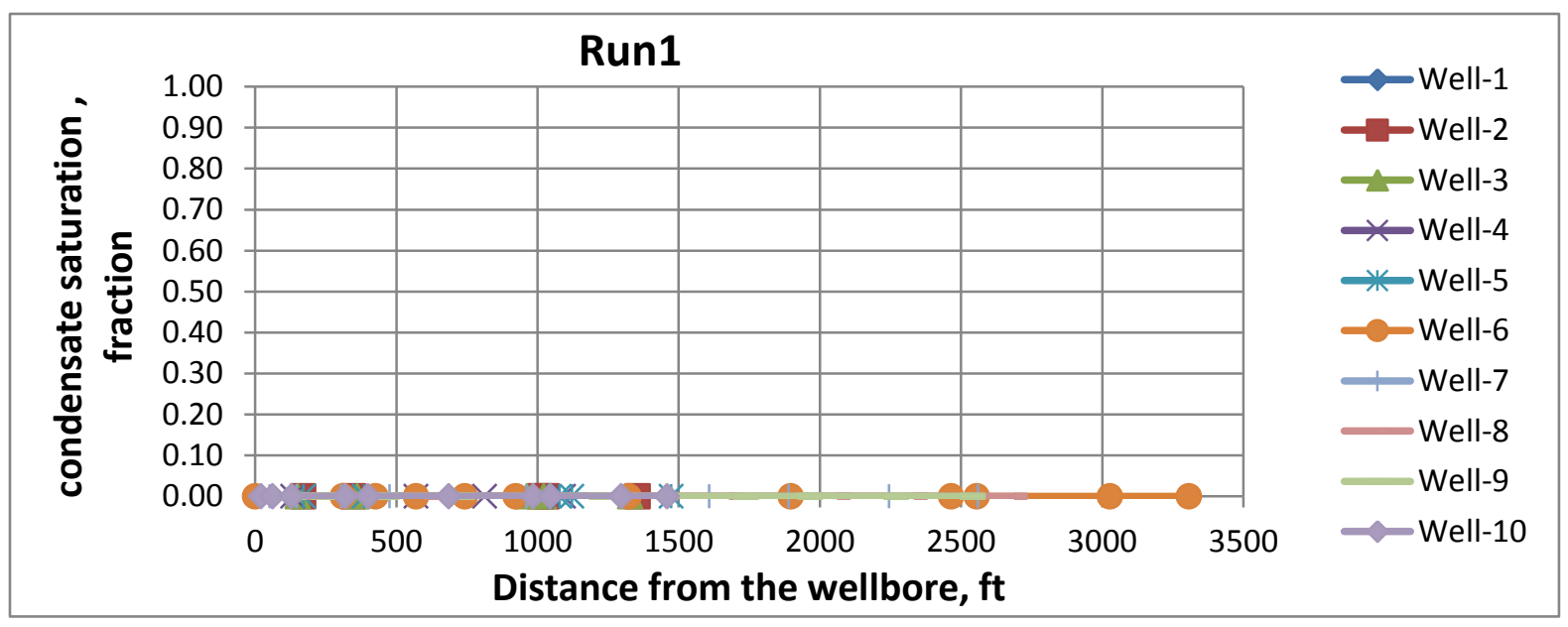

b) : confined case.

Figure (A2. 1 a \& b): Condensate saturation profile around all wells Run1, unconfined case and confined case respectively. 
Comparison between two cases (confined and unconfined) for Run2 (presented in table 10) leads to conclusion as presented in previous case, Run1, where the condensation disappears around all different wells considering the shrinkage in phase envelope due to organic pore wall confinement effects. It is interesting to see that the condensate saturation around the horizontal wells, i.e., 6, 7, 8, 9 and 10, do not follow the discussion on having zones I, II, and III presented earlier in Figure 6. It shows the oscillation of saturation developing high and low concentration zones. Comparing these observations in Run2 with Run1 and keeping in mind the results of our experimental design in Figure 43, it becomes clear that in this case we have higher pore compressibility that has the most significant positive impact on condensation. Therefore first the condensate saturations are higher and second the oscillations, i.e., drop in condensate saturation, occur where we have hydraulic fractures approaching from two nearby horizontal wells.

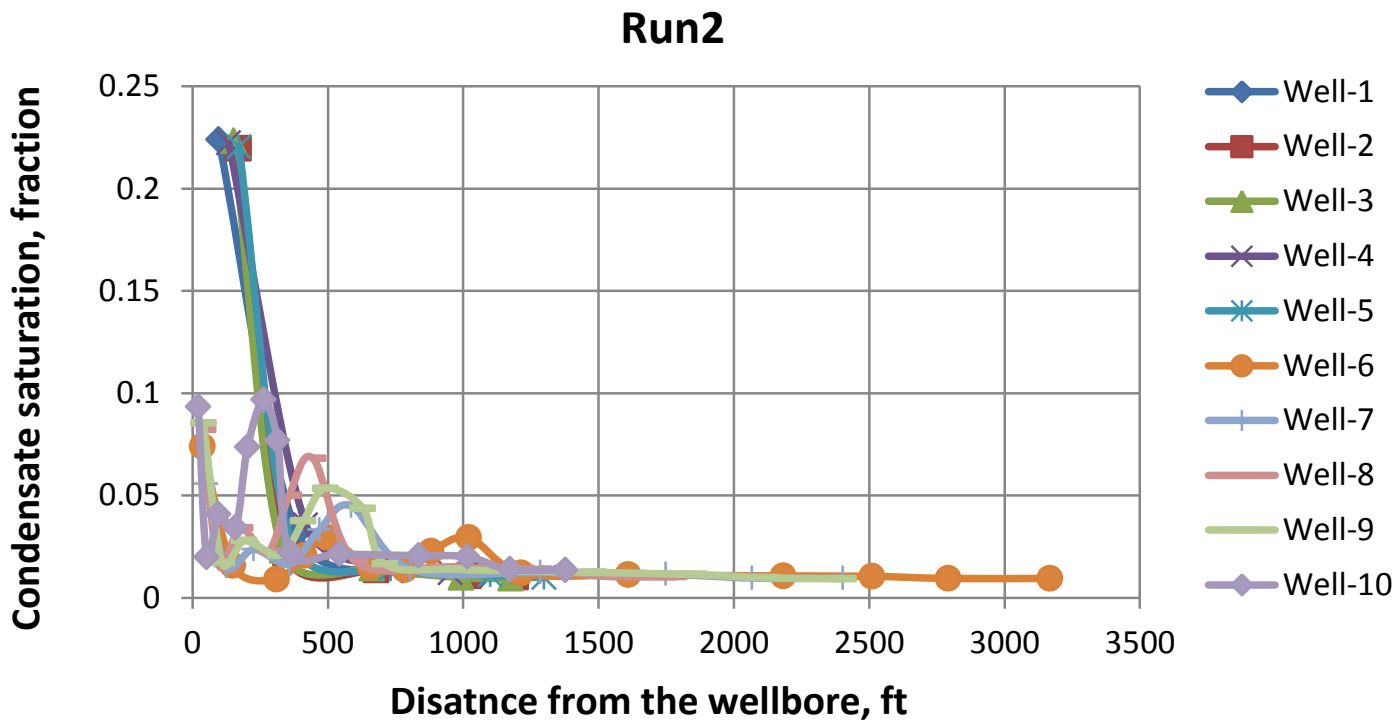

a) : unconfined case. 


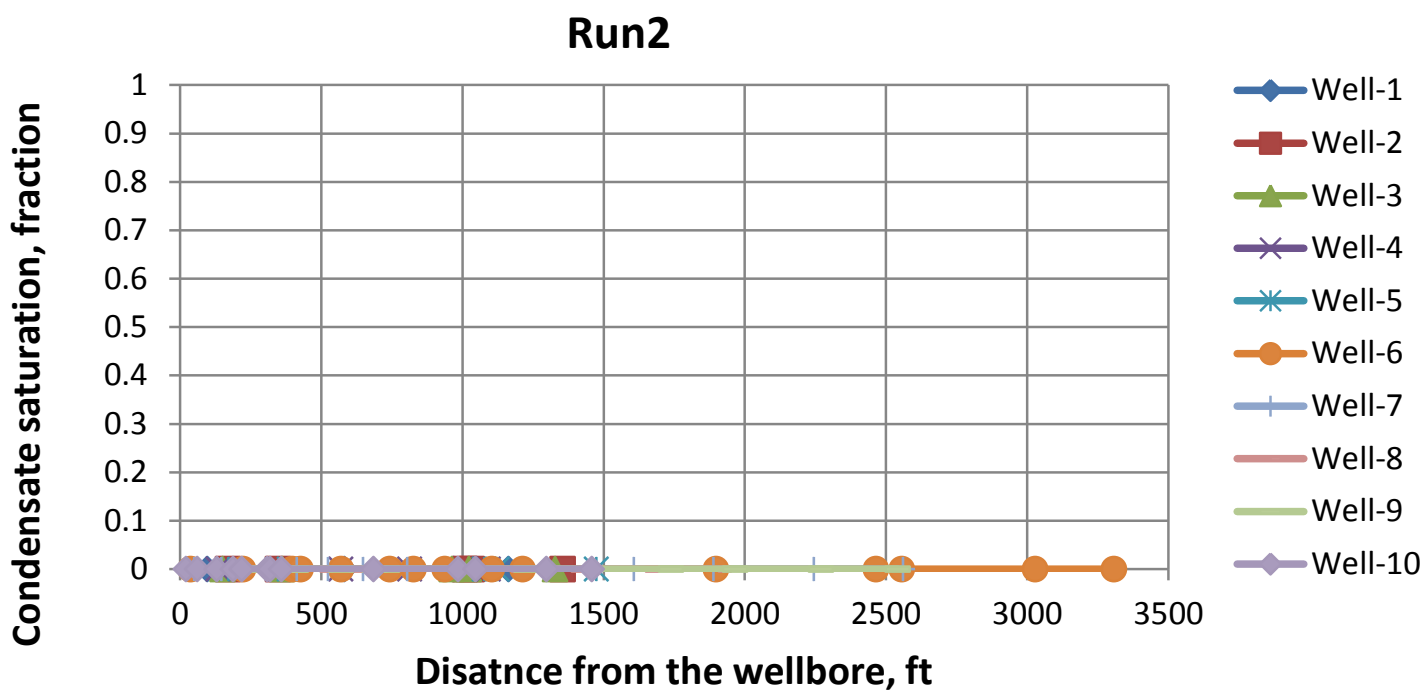

b) : confined case.

Figure (A2. 2 a \&b) : Condensate saturation profile around all wells Run2, unconfined case and confined case respectively.

In Runs 3 and 4 distinct difference exist with previous two runs in that here even considering the organic pore wall confinements we see similar magnitude of the condensation with similar trend to unconfined case. Considering Table 10 (characteristics of each run) it becomes clear that the difference between these runs are: in Run3 and 4 fluid composition is high meaning that we have rich reservoir fluid that leads to much higher liquid drop out and also increasing the quantity of heavy components leads to having much larger two phase envelope. The second parameter that can promote this difference is much lower matrix anisotropy in Run3 and 4 compare to case 1 and 2 that has a negative impact therefore increasing the chance of liquid drop out based on Figures 43 and 44. The oscillations in condensate saturations observed in the case of horizontal wells have been discussed earlier that is due to the presence of hydraulic fractures that produce the condensates at specific locations around the horizontal well generating these oscillations in condensate saturations. 
Run3

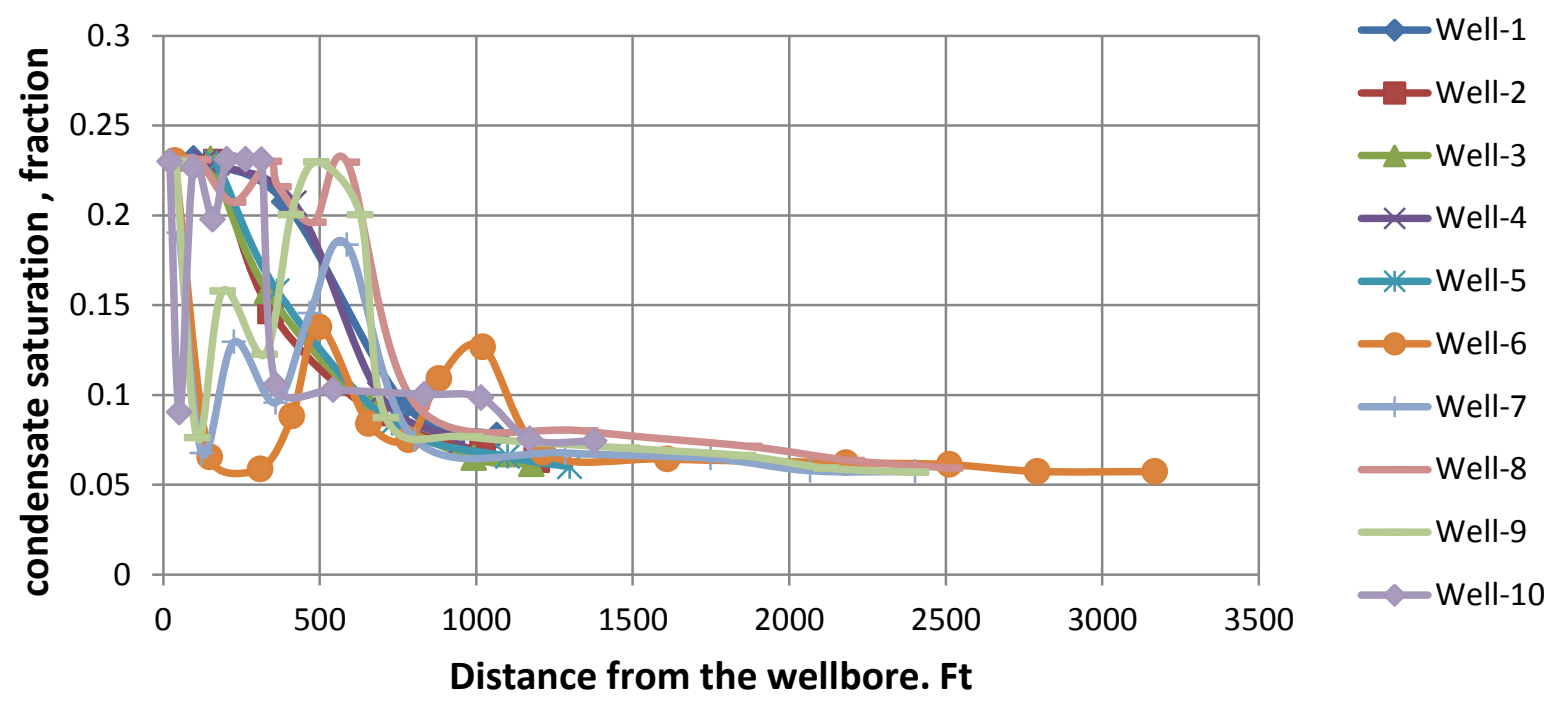

a) : unconfined case

\section{Run3}

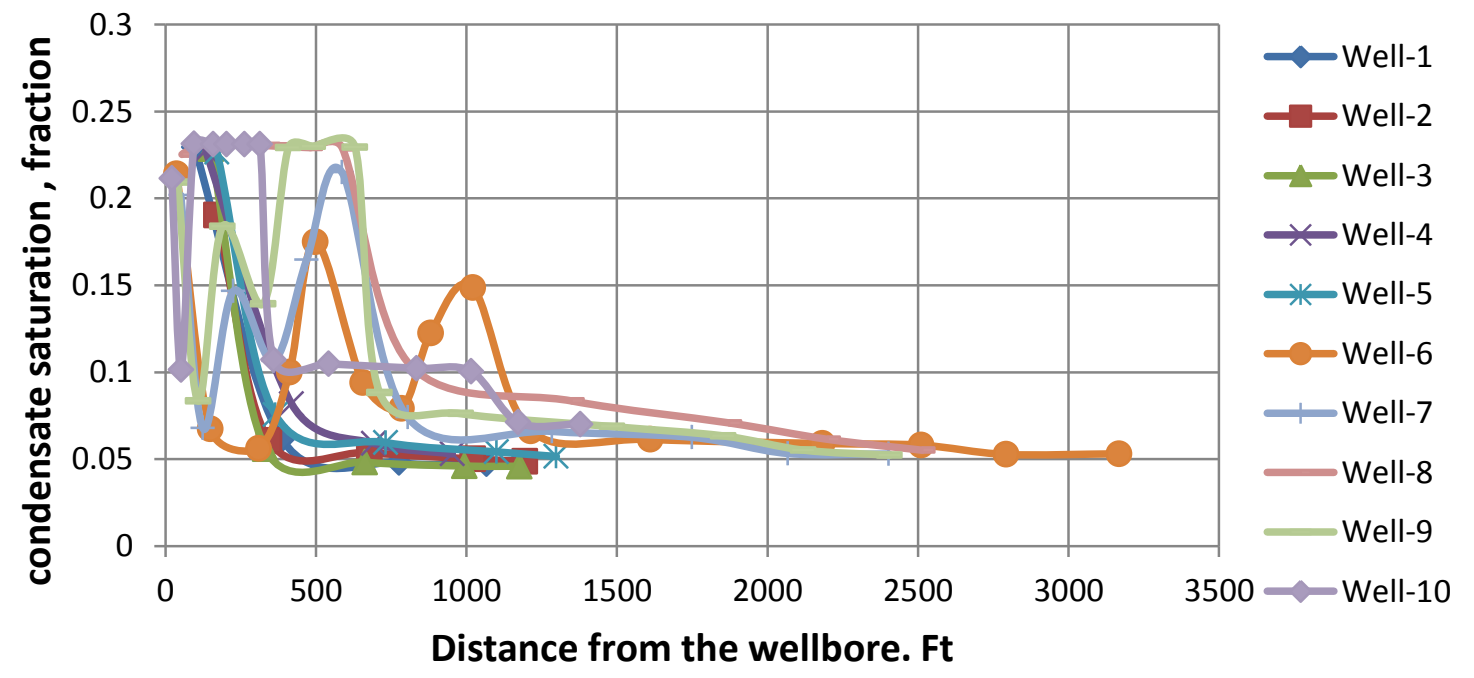

\section{b) : confined case}

Figure (A2. 3 a \&b) : Condensate saturation profile around all wells Run3, unconfined and confined case respectively. 


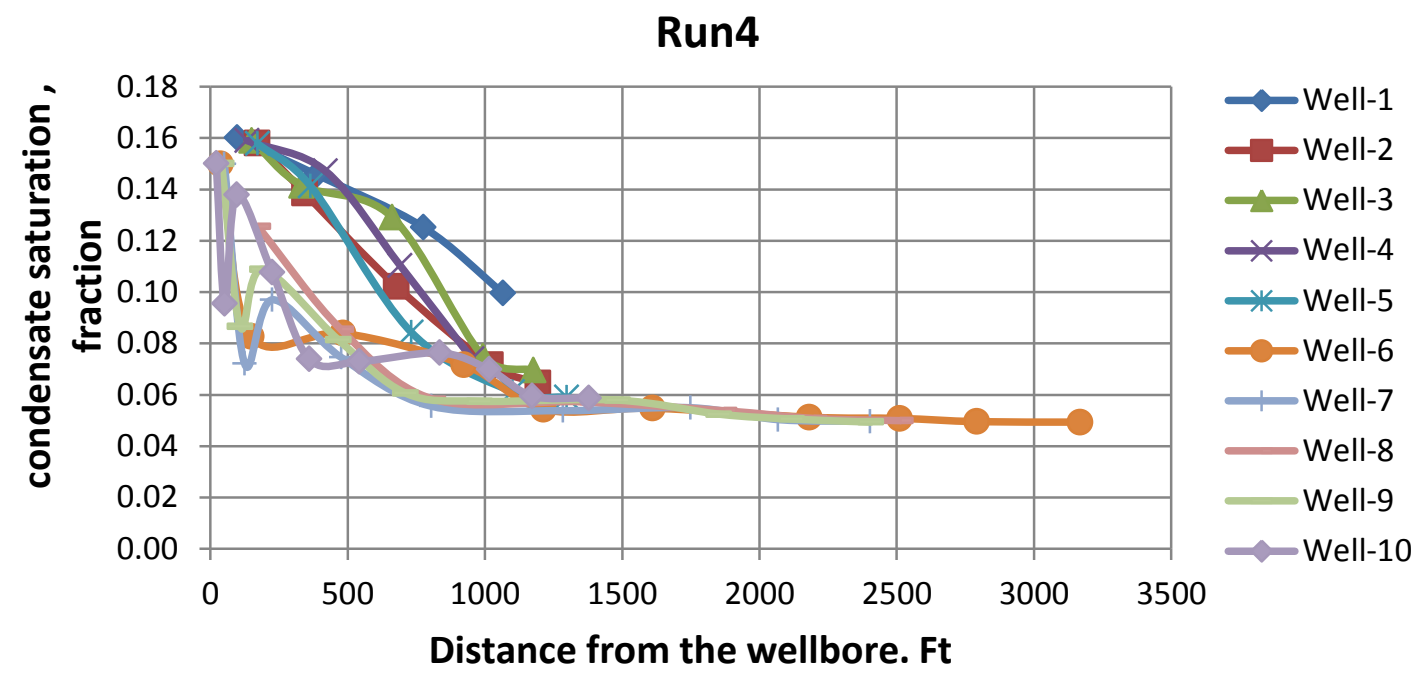

a) : unconfined case.

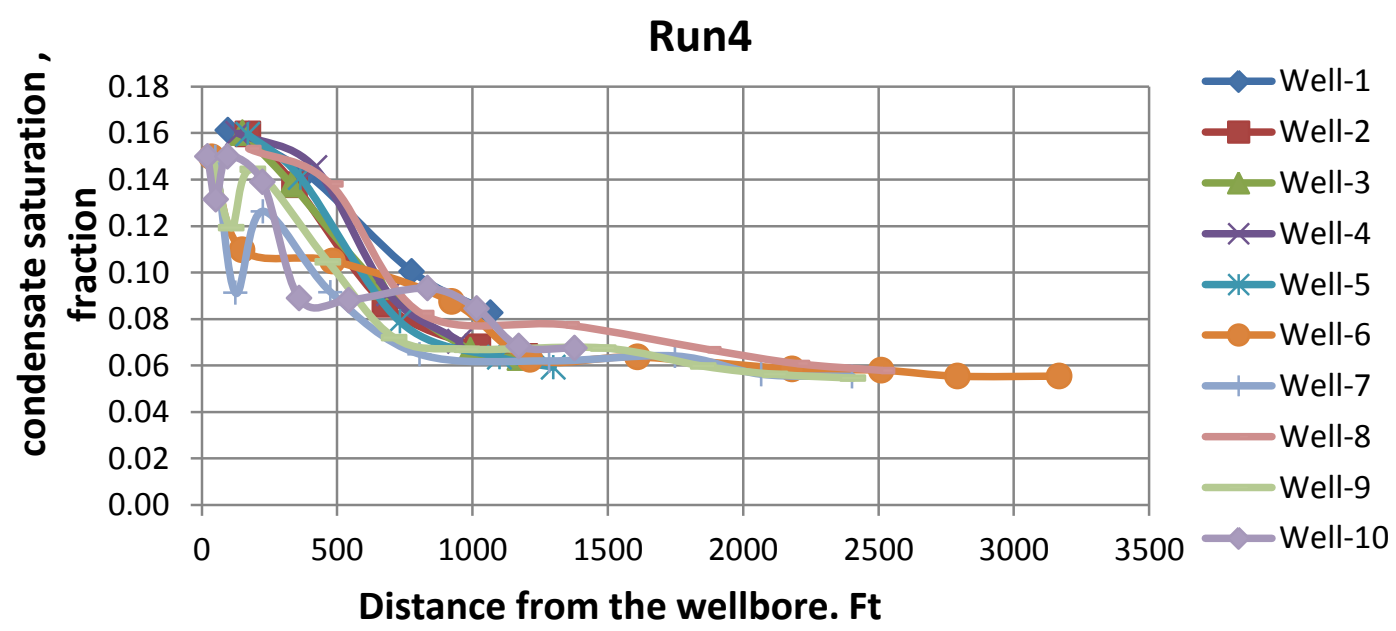

b) : confined case.

Figure (A2. 4 a \& b) : Condensate saturation profile around all wells Run4, unconfined case and confined case respectively..

In Run5 and 6, similar trends as Run1 and 2 have been observed, embracing the effect of organic pore wall confinements on phase envelope leading to elimination of condensation. We also do not see that aggressive oscillation in condensate saturations due to the fact that in Run5 and 6 in contrary to Run3 and 4 the reservoir fluid is not rich 
that leads to less condensation, therefore the effect of hydraulic fractures are not as pronounced as Run3 and 4.

\section{Run5}

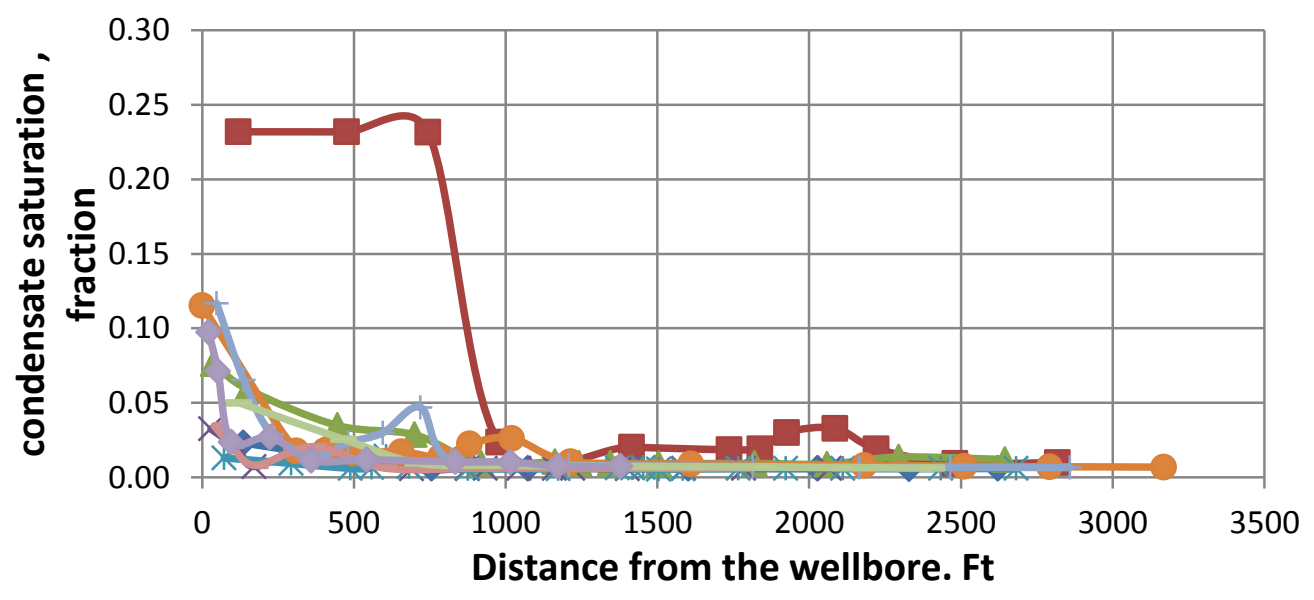

$\longrightarrow$ Well-1

- Well-2

$\sim$ Well-3

$\because$ Well-4

*Well-5

- Well-6

+ Well-7

Well-8

Well-9

$\longrightarrow$ Well-10

\section{a) unconfined case.}

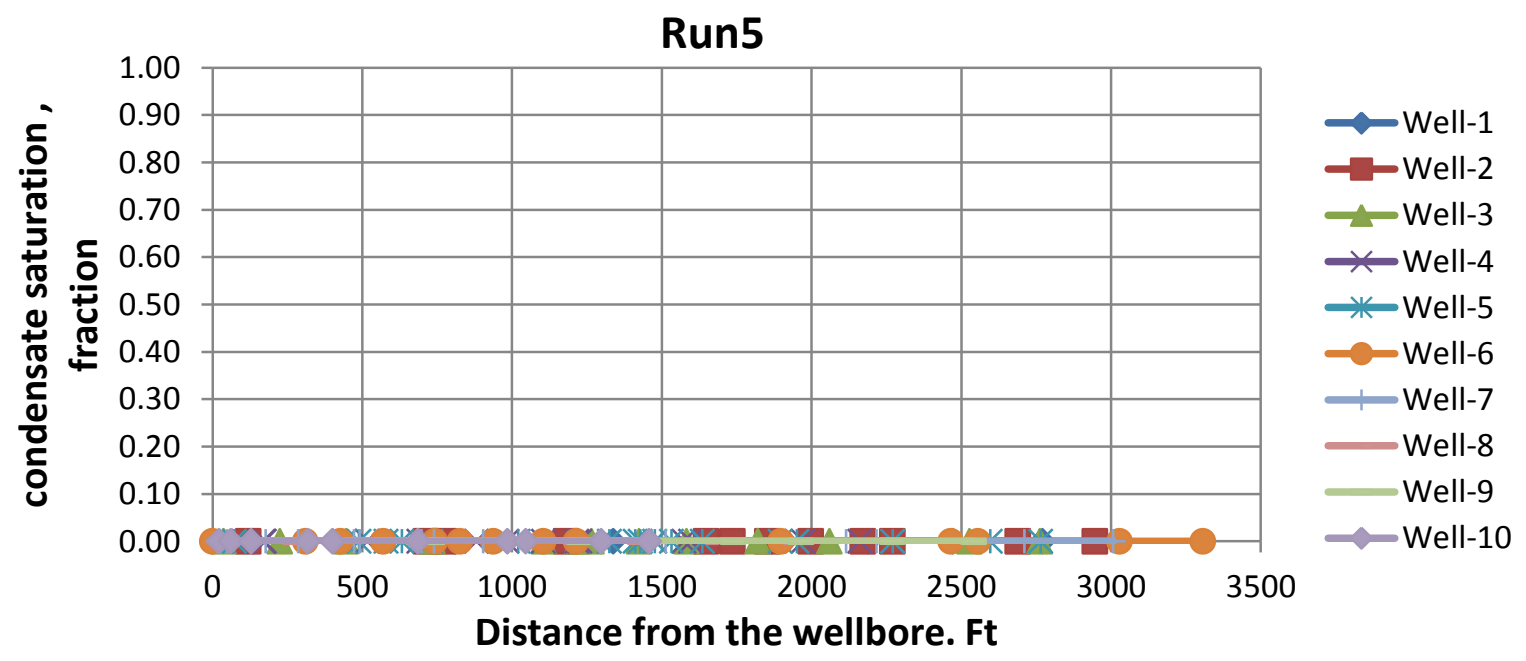

\section{b) confined case.}

Figure (A2. $5 \mathrm{a} \& \mathrm{~b}$ ) : Condensate saturation profile around all wells Run5, unconfined case. and confined case respectively. 


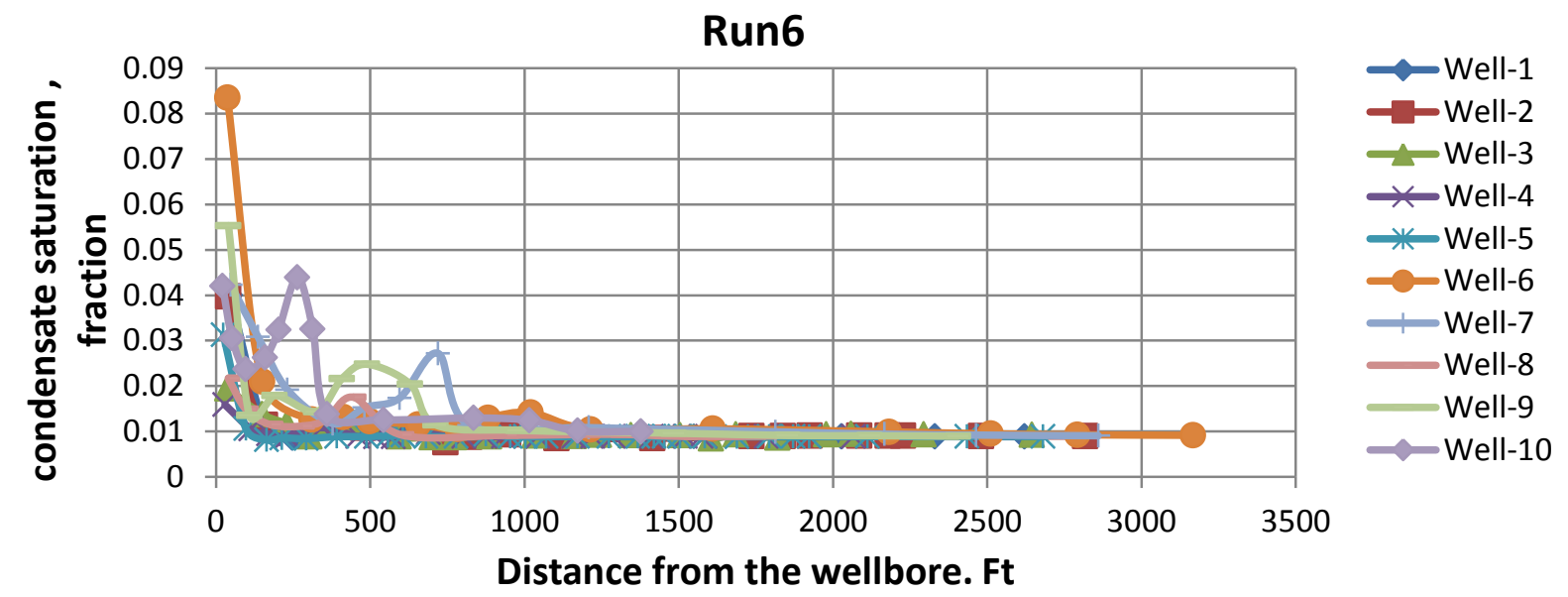

\section{a) : unconfined case.}

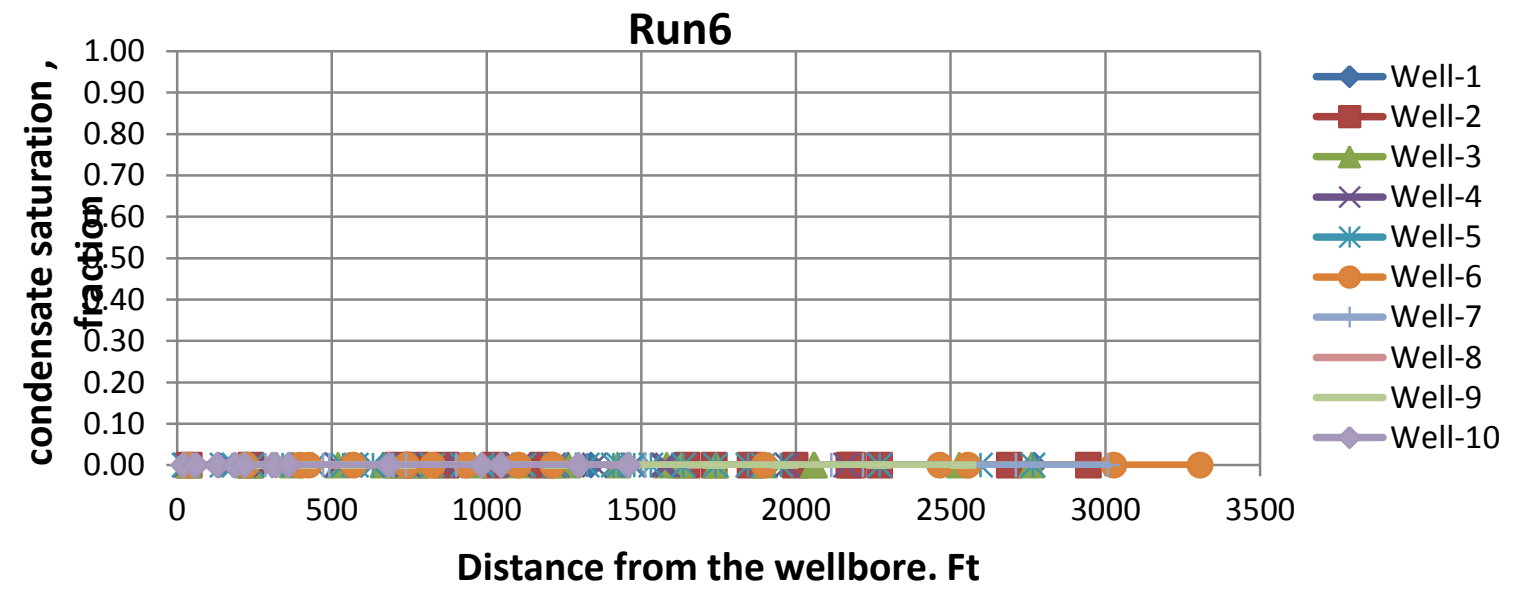

\section{b) : confined case.}

Figure (A2. 6 a \&b ) : Condensate saturation profile around all wells Run6, unconfined case and confined case respectively.

In Run7 for unconfined case 4 parameters are in favor of decreasing the mount of condensation, i.e., low pressure gradient, low number of hydraulic fractures, low anisotropy and low number of horizontal wells, that seems to have more pronounced effect on condensation that the parameters in favor of condensation. In the case of confined case the organic pore wall effect is dominated eliminating the condensation. 


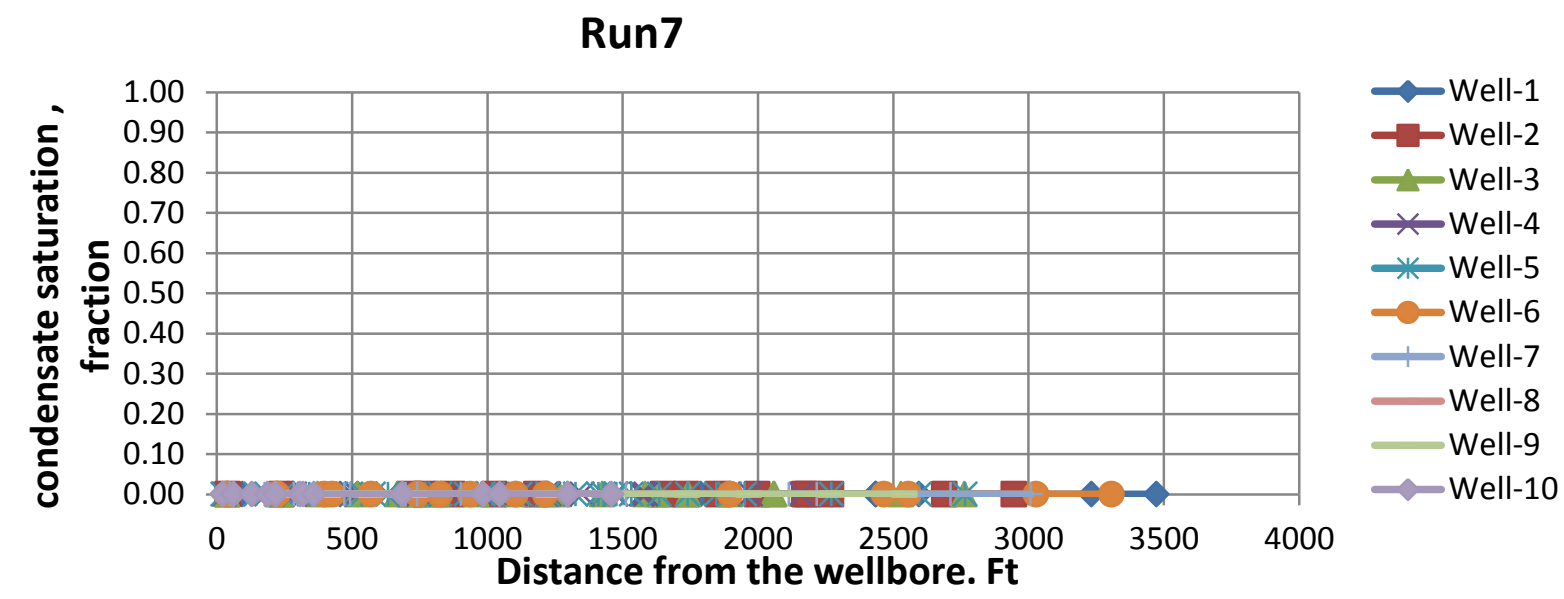

\section{b) : unconfined case.}

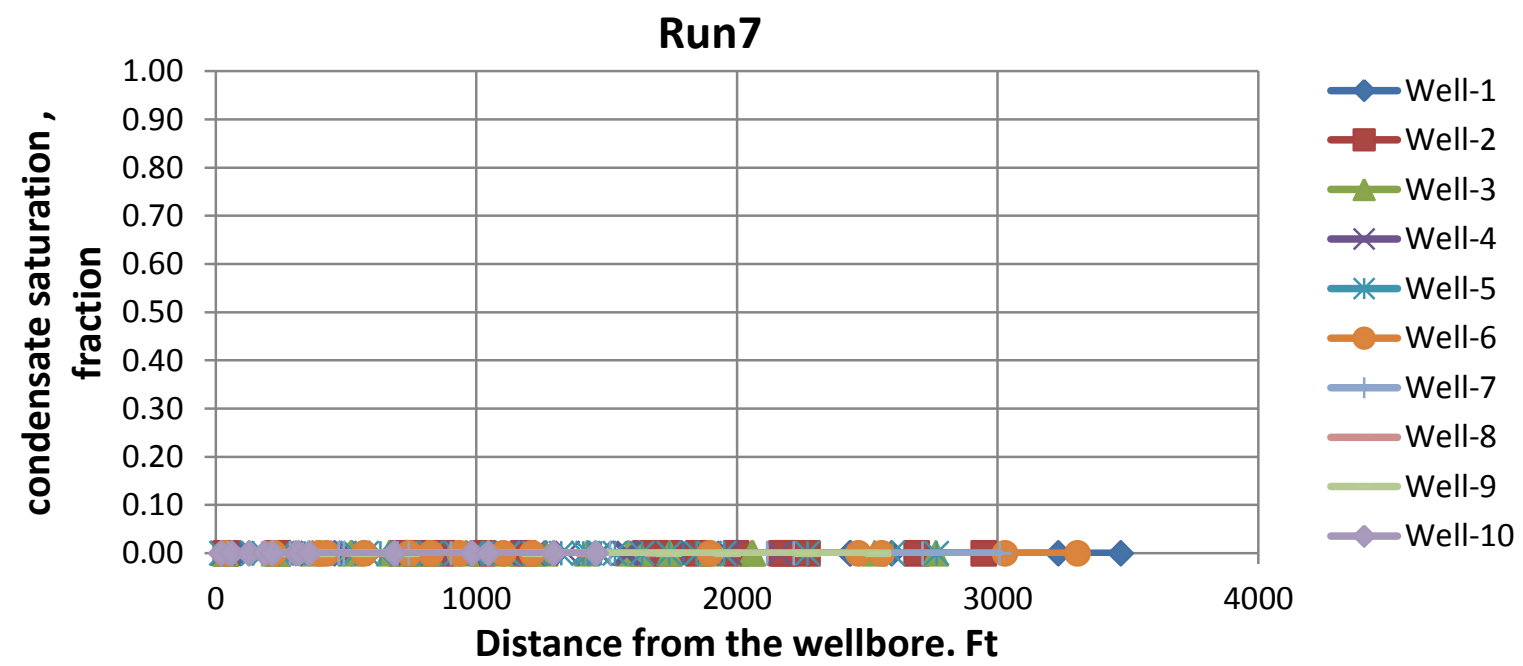

\section{b) : confined case.}

Figure (A2. 7 a \&b) : Condensate saturation profile around all wells Run7, unconfined case and confined case respectively.

Figure (A2. 8) displays run (8) which depict similar behavior in case of high condensation saturation in both confined and unconfined cases and also oscillation of condensation saturation around horizontal wells due to hydraulic fractures as discussed earlier in Run 3. However, there is one more interesting observation here and that is the importance of parameters with positive impact on condensation in compare with those having negative impact on condensation. In this Run all parameters having the high 
values demonstrating clear view of the competition between positive and negative impacts on increasing or decreasing condensation saturation which leads to overcome of positive impacts. This conclusion is in agreement with Figures 43 and 44 discussed earlier.

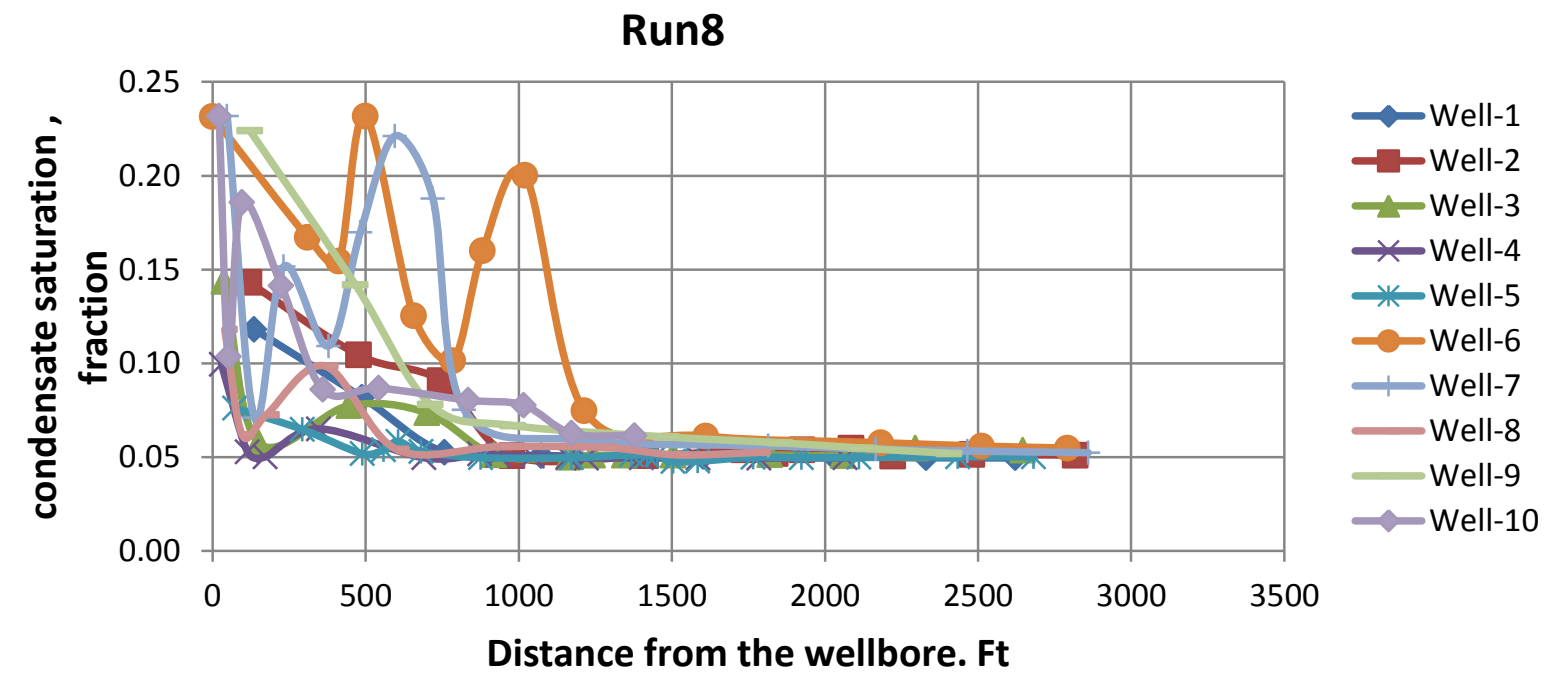

a): unconfined case

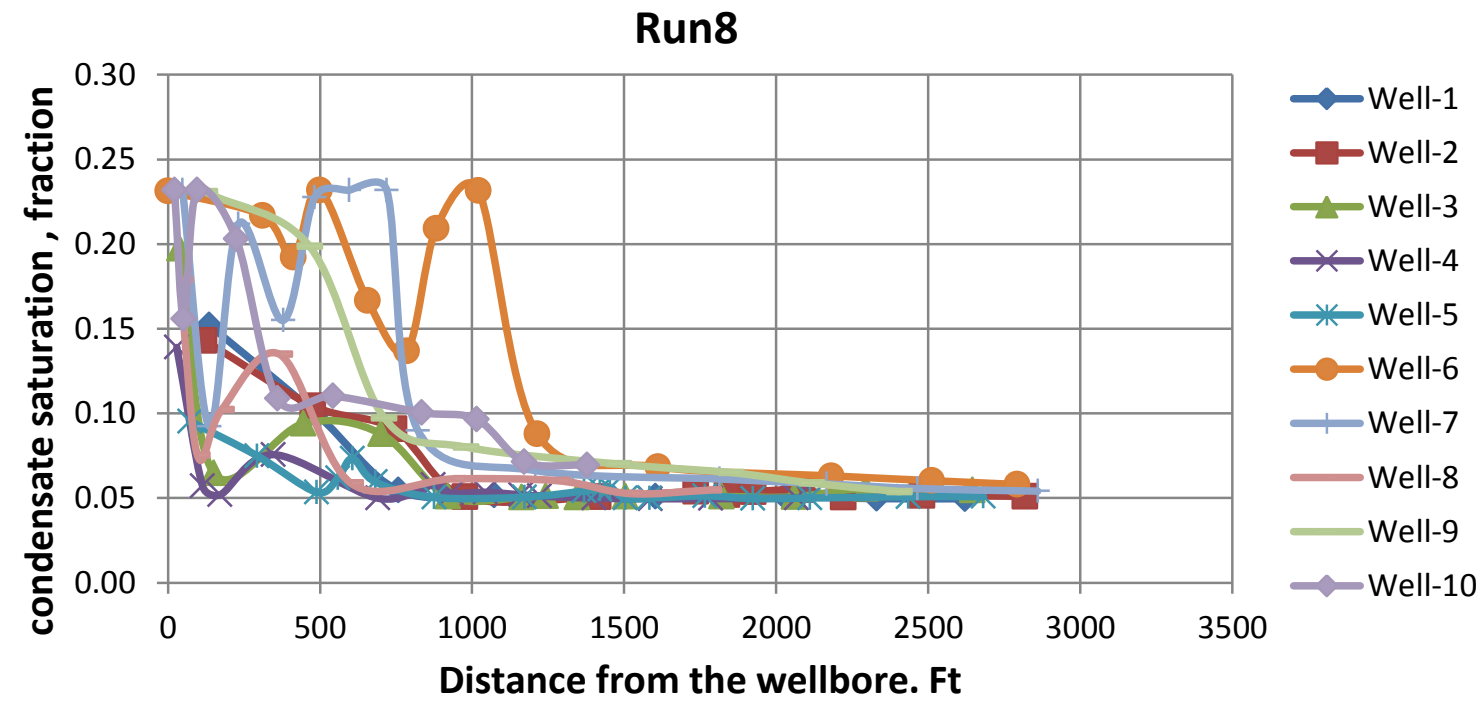

\section{b): confined case}

Figure (A2. 8 a \& b ) : Condensate saturation profile around all wells Run8, unconfined case and confined case respectively. 


\section{Appendix B: Correlations Used In This Study:}

\section{B.1: Determination of the Critical Properties Deviation Due To Pore Wall Confinement:}

This approach was developed by Zaraagoicoechea (2004) based on the Helmholz free energy the Vander Waals equation of state to establish a relationship between the critical temperature shifting of a fluid flowing inside pores due to relative molecular diameter to the limited space.

Helmholz free energy is established as follows:

$$
A=f(T)-N k T \ln \left(V-N b \sigma^{3}\right)+2 \frac{N^{2}}{V} \varepsilon \sigma^{3}\left(-\frac{a}{2}+\frac{c 1}{\sqrt{A p}}+\frac{c 2}{A p}\right)
$$

Where the constants can be defined as: $b=\frac{2 \pi}{3} \quad, \quad a=\frac{16 \pi}{9} \quad, \quad \mathrm{c}_{1}=4.6571$ and $\mathrm{C}_{2}=-2.1185$. And the geometry of the pore as: $A p=\pi\left(\frac{r p}{\sigma}\right)^{2}, \quad V=\mathrm{A}_{\mathrm{p}}{ }^{*} \mathrm{~L}_{\mathrm{z}}$

Since the axial pressure is defined as a function of the fluid temperature inside the pore and the pore area in the relationship below:

$$
P z Z=-\left(\frac{1}{A p}\right)\left(\frac{\partial A}{\partial L z}\right) T, A p
$$

Then since the reduced axial pressure can be stated as: $P^{*}{ }_{Z Z}=\frac{P_{Z Z} \sigma^{3}}{\varepsilon}$

Using Van der waals parameters, this can be expressed as:

$$
P_{Z Z}^{*}=\frac{T^{*}}{v^{*}-b}-\frac{a-2\left(\frac{c_{1}}{\sqrt{A_{p}}}\right)-2\left(\frac{c_{2}}{A_{p}}\right)}{v^{* 2}}
$$


Where: $T^{*}=\frac{k T}{\varepsilon} \quad v^{*}=\left(\frac{V}{N}\right) \sigma^{3}$

The correction of VW-EOS for the Pore walls confinement was by adjusting the equation parameters established from the experiment data:

$$
P_{Z Z}^{*}=\frac{T^{*}}{v^{*}-b_{a d j}}-\frac{\left(\frac{a_{a d j}}{a}\right)\left(a-\left(\frac{2 c_{1}}{\sqrt{A_{p}}}\right)-\left(\frac{2 c_{2}}{A_{p}}\right)\right.}{v^{* 2}}
$$

Since the bulk values of the single components were calculated using the following proposed equation:

$T_{C}{ }^{*}=\frac{8 a_{a d j}}{27 b_{a d j}}$

$P_{C}^{*}=\frac{8 a_{a d j}}{27 b^{2} a d j}$

$V_{C}^{*}=3 b_{a d j}$

This correction was applied to those bulk parameters to express the fluid components inside the pore space as described in the flowing equations:

$$
\begin{aligned}
& T^{*}{ }_{c p}=\frac{8}{27 b_{a d j}} \frac{a_{a d j}}{a}\left(a-\left(2 c_{1} / \sqrt{ } A_{P}\right)-\left(\frac{2 c_{2}}{A_{p}}\right)\right. \\
& P^{*}{ }_{c p}=\frac{\left(a_{a d j} / a\right)\left(a-\left(2 c_{1} / \sqrt{ } A_{P}\right)-\left(2 c_{2} / A_{p}\right)\right.}{27 b_{a d j}} \\
& v^{*}{ }_{c p}=27 b_{a d j}
\end{aligned}
$$


By using the two sets of the fluid flow in bulk and pore space the relationship between the temperature deviation and the molecular to pore diameter was established and expressed as follows:

$$
\frac{T^{*}{ }^{-}-T^{*} c p}{T^{*} c}=2 \frac{c 1}{\sqrt{\pi a}}\left(\frac{\sigma}{r p}\right)+2 \frac{c 2}{\pi a}\left(\frac{\sigma}{r p}\right)^{2} .
$$

Substituting the constants with their actual values, the result is the following equation for the temperature shifting:

$$
\frac{T_{c}-T^{*} c p}{T_{c}}=0.9409\left(\frac{\sigma}{r p}\right)-0.2415\left(\frac{\sigma}{r p}\right)^{2}
$$

Analogous to the temperature effect by the pore confinement, the pressure deviation is also considered to reduce due to the confinement but due to lack of experiments data. It was proposed by the following equation:

$$
\frac{P_{c}-P_{C p}}{P_{c}}=0.9409\left(\frac{\sigma}{r_{p}}\right)-0.2415\left(\frac{\sigma}{r_{p}}\right)^{2}
$$




\section{B.2: Viscosity Correlations Of Pure Substances: (JST)}

The viscosity correlation used in this thesis is the the Jossi, Stiel and Thodos (JST) method proposed by Fong and Ngheim 1980, integrated in the WinProp application by the CMG simulator. This was the most popular in the oil and gas industry in which the fluid viscosity measurement was performed under low conditions. The correlation was given by the following equation:

$$
\left[\left(\mu-\mu^{*}\right) \xi-10^{-4}\right]^{\frac{1}{4}}=a_{0}+a_{1} \rho_{r}+a_{2} \rho_{r}{ }^{2}+a_{3} \rho_{r}{ }^{3}+a_{4} \rho_{r}{ }^{4} \ldots \ldots \ldots \ldots \ldots \ldots \ldots \ldots . . .2 .1
$$

Since $\mu^{*}$ represents the low pressure viscosity, it can be calculated from the following equation:

$\mu^{*}=\frac{\sum_{i}\left(X_{i} \mu_{i}^{*} M_{W i} i^{1 / 2}\right)}{\sum_{i}\left(X_{i} M_{W i^{1 / 2}}\right)}$

And the viscosity parameter $(\xi)$ is given by this formula:

$$
\xi=\frac{\left[\sum_{i} X_{i} T_{c i}\right]^{1 / 6}}{\left[\sum_{i} X_{i} M W_{i}\right]^{1 / 2}\left[\sum_{i} X_{i} P_{c i}\right]^{2 / 3}}
$$

The reduced density was given by:

$$
\rho_{r}=\rho V_{c m}=\rho\left[\sum_{i=1}^{n} X_{i} V^{\alpha} C m i\right]^{1 / \alpha}
$$


For a single component inside the pores viscosity is calculated using the formula proposed by JST (1961).

$\mu_{i}^{*} \xi_{i}=\left[4.610 T_{r i}{ }^{0.618}-2.04 \exp \left(-0.449 T_{r i}\right)+1.94 \exp \left(-4.058 T_{r i}\right)-0.1\right] * 10^{-4}$

Where: $\xi_{i}=T_{c i} i^{1 / 6} M W i^{-1 / 2} P_{c i} i^{-2 / 3} \quad$ and $\quad T_{r i}=\frac{T}{T_{c i}}$

Finally: The general dimensionless constants in the proposed correlation are listed below:

$$
\begin{aligned}
& a=1 \\
& a_{0}=1.0230 \times 10^{-1} \\
& a_{1}=2.3364 \times 10^{-2} \\
& a_{2}=5.8533 \times 10^{-2} \\
& a_{3}=-4.0758 \times 10^{-2} \\
& a_{4}=9.3324 \times 10^{-3}
\end{aligned}
$$

